\title{
Comparing Measures of Physical Activity Intensity, Duration, and Frequency Using Receiver Operator Characteristic Curve Analyses
}

\author{
Abigail M. Nehrkorn-Bailey \\ West Virginia University, abnehrkorn@mix.wvu.edu
}

Follow this and additional works at: https://researchrepository.wvu.edu/etd

Part of the Developmental Psychology Commons, and the Quantitative Psychology Commons

\footnotetext{
Recommended Citation

Nehrkorn-Bailey, Abigail M., "Comparing Measures of Physical Activity Intensity, Duration, and Frequency Using Receiver Operator Characteristic Curve Analyses" (2019). Graduate Theses, Dissertations, and Problem Reports. 3896.

https://researchrepository.wvu.edu/etd/3896

This Dissertation is protected by copyright and/or related rights. It has been brought to you by the The Research Repository @ WVU with permission from the rights-holder(s). You are free to use this Dissertation in any way that is permitted by the copyright and related rights legislation that applies to your use. For other uses you must obtain permission from the rights-holder(s) directly, unless additional rights are indicated by a Creative Commons license in the record and/ or on the work itself. This Dissertation has been accepted for inclusion in WVU Graduate Theses, Dissertations, and Problem Reports collection by an authorized administrator of The Research Repository @ WVU. For more information, please contact researchrepository@mail.wvu.edu.
} 
Comparing Measures of Physical Activity Intensity, Duration, and Frequency Using Receiver Operator Characteristic Curve Analyses

\author{
Abigail M. Nehrkorn-Bailey, MS \\ Dissertation submitted \\ to the Eberly College of Arts and Sciences \\ at West Virginia University \\ in partial fulfillment of the requirements for the degree of \\ Doctor of Philosophy in \\ Psychology
}

Julie Hicks Patrick, Ph.D., Chair

Kristina Hash, Ph.D.

Kevin Larkin, Ph.D.

Aaron Metzger, Ph.D.

Department of Psychology

Morgantown, West Virginia

2019

Keywords: physical activity, validity, activity duration, activity intensity, activity frequency Copyright 2019 Abigail M. Nehrkorn-Bailey 


\begin{abstract}
Comparing Measures of Physical Activity Intensity, Duration, and Frequency Using Receiver

Operator Characteristic Curve Analyses
\end{abstract}

Abigail M. Nehrkorn-Bailey

The United States Department of Health and Human Services (HHS) recommends adults to engage in weekly moderate- or vigorous-intensity physical activity based on its association with various physical and psychological health benefits (HHS, 2008; Schoenborn, Adams, \& Peregoy, 2013). These physical activity recommendations contain important information for three physical activity components: intensity, frequency, and duration. The current physical activity literature contains gaps, with a lack of specificity for which components are being studied. Although some of the literature does describe the physical activity components, there are many discrepancies in the level of agreement across subjective and objective measures, along with the same basic analyses being utilized across studies. With data from 56 adults (Mage = 43.2, $S D=16.5$ ), receiver operator characteristic (ROC) curve analyses were conducted for two weeks' data to assess the agreement between a one-week recall questionnaire, daily diary accounts, and accelerometer data for the three physical activity components (intensity, frequency, and duration). For intensity and duration of physical activity, the worst agreement existed between the accelerometer and questionnaire, with the area under the curve (AUC) indicating chance agreement at best $(\mathrm{AUC}=0.447-0.598)$; the accelerometer and daily diary measures generally exhibited chance-to-poor agreement $(\mathrm{AUC}=0.331-0.671)$; and the daily diary and questionnaire showed the largest range from chance-to-good agreement (AUC $=0.482$ 0.794). The strongest agreement was shown for frequency of physical activity, especially for mild-intensity physical activity $(\mathrm{AUC}=0.836$ ) at Week 2 . Across moderate-to-vigorous-, mild-, and sedentary-intensity behaviors, participants experienced the worst agreement for sedentary behaviors (AUC $=0.331-0.686$ ), with most of the sedentary-specific intensity, frequency, and duration AUC scores showing chance agreement. The current study significantly expands and adds to the literature by focusing on all three physical activity components across moderate-tovigorous-, mild-, and sedentary-intensity activities, as well as utilizing advanced analyses to assess the agreement between two subjective measures and one objective measure. The findings highlight the importance of educating adults about the physical activity components, different intensities, and methods of tracking and reporting activity. 


\section{Acknowledgements}

The support I have received from my mentor, professors, friends, and family has propelled me to this milestone. Their positivity, encouragement, and knowledge will never be forgotten. First, I would like to thank my mentor Dr. Julie Hicks Patrick. Dr. Patrick's instruction carried through to my writing, research, and knowledge of the field. However, her mentoring and support also helped me to strengthen my passion for the field and to realize the vision I had for my academic future. Connectedly, I am grateful to my many professors, graduate school friends, and lab members for their constant encouragement. I also want to thank my Midwestern friends, family, and godson Leo for their love and support from afar. Special thanks go to my dad Steve Nehrkorn for his constant belief in me and my continued endeavors. A special thank you also goes to my sister Amanda Nehrkorn Jacobson. As your prodigy from a young age, you've always believed in me. Without your constant love, support, and visits, I would not be writing this acknowledgement. Dad and Amanda, your belief in me has paid off! Lastly, I want to thank my husband Zach Bailey. Zach, your love and support has helped me to achieve so much. Thank you for always supporting me and my passion. 


\section{Table of Contents}

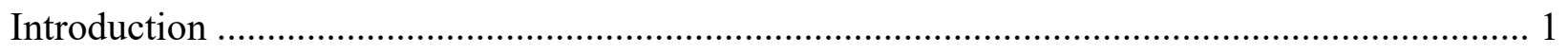

Physical Activity Recommendations ........................................................................... 2

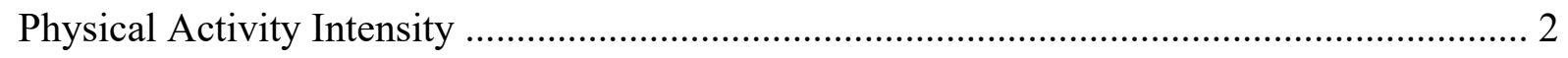

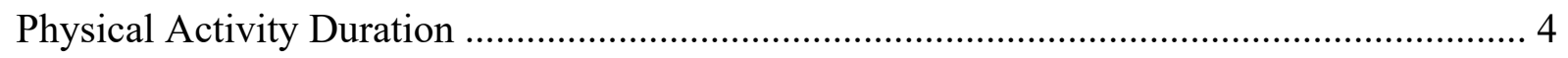

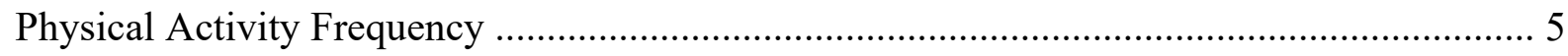

Subjective Measures of Physical Activity .................................................................... 6

Objective Measures of Physical Activity ....................................................................... 7

Validity of Physical Activity Measures …............................................................... 8

Individual Differences in Accurately Reporting Physical Activity ................................. 16

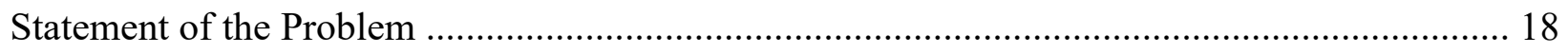

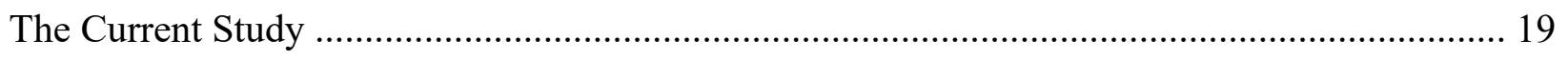

Research Questions and Hypotheses ....................................................................... 19

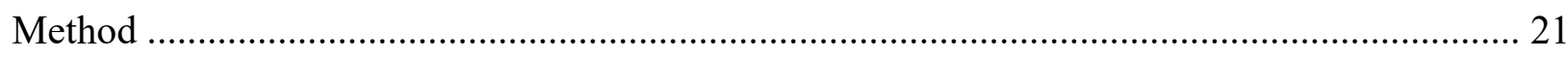

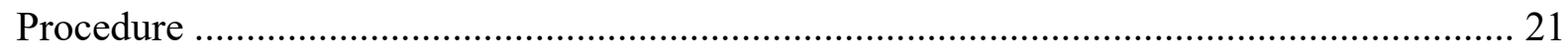

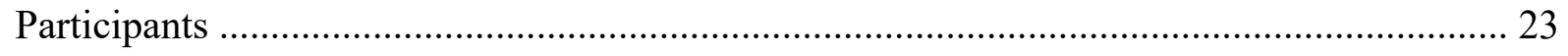

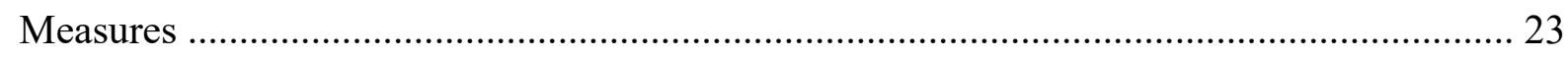

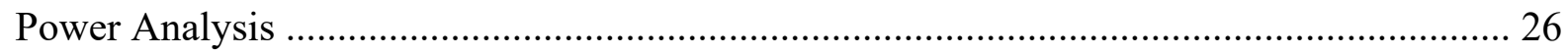

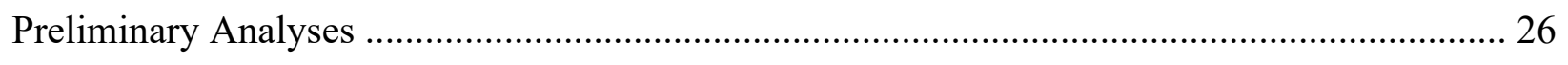

Receiver Operator Characteristic Curve Analyses ........................................................ 27

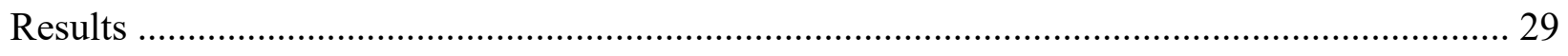

Research Question 1: Agreement for Intensity, Frequency, and Duration ......................... 29

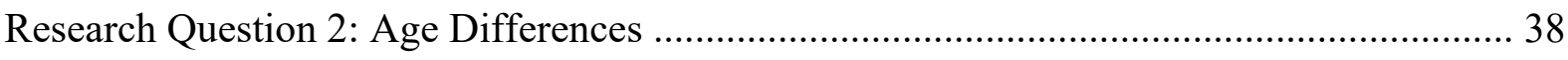




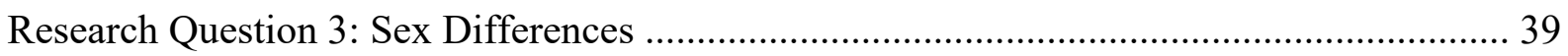

Research Question 4: Intrinsic Motivation Differences ........................................................ 41

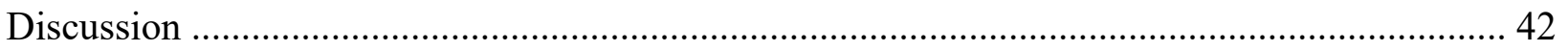

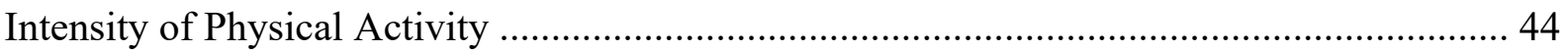

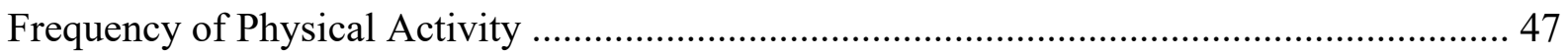

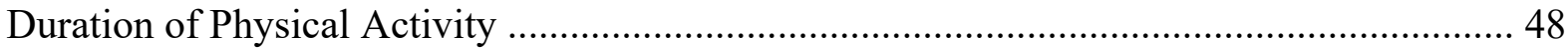

Group Differences in Reporting Intensity, Frequency, and Duration ................................... 50

Statistical Analyses to Study Intensity, Frequency, and Duration .......................................... 55

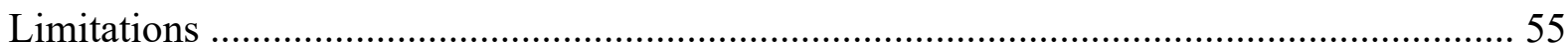

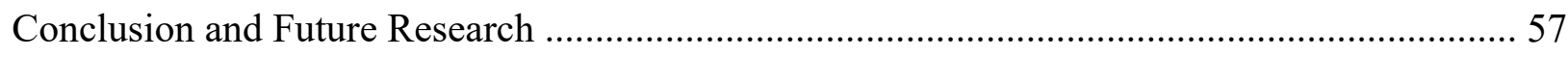

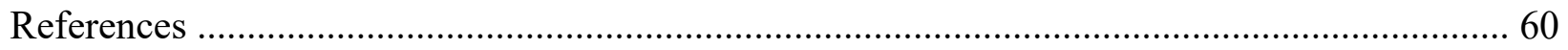

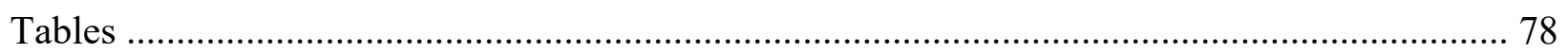

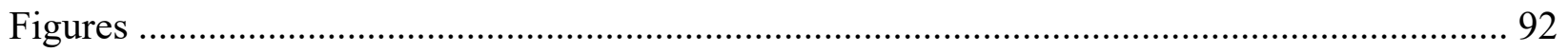

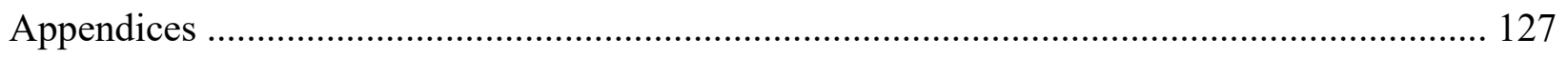

Appendix A: ABCD Prescreen and Introduction to Study................................................. 127

Appendix B: The Goal-Striving Reasons Index (Ehrlich, 2012) …………………............ 128

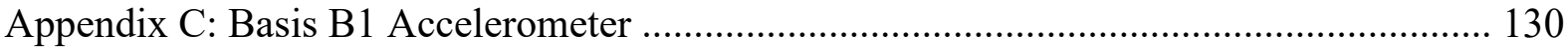

Appendix D: The Godin Leisure-Time Exercise Questionnaire (Godin \& Shephard,

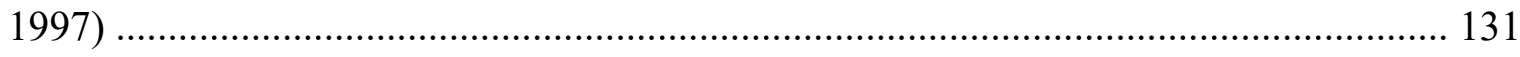

Appendix E: Daily Diary Assessment …………………............................................... 132

Appendix F: Research Questions and Hypotheses .......................................................... 133 


\section{List of Tables}

Table 1: Intensity, frequency, and duration findings from validation studies ......................... 78

Table 2: Mean activity scores (intensity) ......................................................................... 79

Table 3: Mean number of days (frequency) engaged in physical activity ............................... 80

Table 4: Mean number of minutes (duration) engaged in physical activity ............................ 81

Table 5: Intraclass correlations for all measures and intensities ......................................... 82

Table 6: ROC curve analysis AUC statistics for activity scores (intensity) ............................ 83

Table 7: ROC curve analysis AUC statistics for frequency .................................................. 84

Table 8: ROC curve analysis AUC statistics for duration .............................................. 85

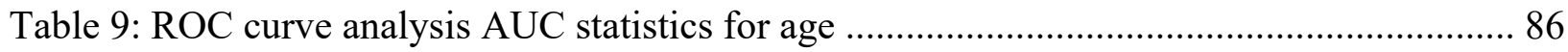

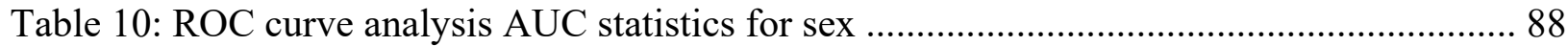

Table 11: ROC curve analysis AUC statistics for intrinsic motivation ................................. 90 


\section{List of Figures}

Figure 1: Flowchart detailing the process of inclusion into the study

Figure 2: Intensity ROC curve analysis for Week 2 moderate-to-vigorous-intensity (accelerometer and GLTEQ) 93

Figure 3: Intensity ROC curve analysis for Week 2 moderate-to-vigorous-intensity (accelerometer and daily diary) 94

Figure 4: Intensity ROC curve analysis for Week 2 moderate-to-vigorous-intensity (daily diary and GLTEQ) 95

Figure 5: Intensity ROC curve analysis for Week 2 mild-intensity (accelerometer and

GLTEQ) 96

Figure 6: Intensity ROC curve analysis for Week 2 mild-intensity (accelerometer and daily diary)

Figure 7: Intensity ROC curve analysis for Week 2 mild-intensity (daily diary and GLTEQ) 98

Figure 8: Intensity ROC curve analysis for Week 2 sedentariness (accelerometer and daily diary) 99

Figure 9: Intensity ROC curve analysis for Week 3 moderate-to-vigorous-intensity (accelerometer and GLTEQ) 100

Figure 10: Intensity ROC curve analysis for Week 3 moderate-to-vigorous-intensity (accelerometer and daily diary) 101

Figure 11: Intensity ROC curve analysis for Week 3 moderate-to-vigorous-intensity (daily diary and GLTEQ) 102

Figure 12: Intensity ROC curve analysis for Week 3 mild-intensity (accelerometer and 
GLTEQ)

Figure 13: Intensity ROC curve analysis for Week 3 mild-intensity (accelerometer and daily diary) 104

Figure 14: Intensity ROC curve analysis for Week 3 mild-intensity (daily diary and

GLTEQ) 105

Figure 15: Intensity ROC curve analysis for Week 3 sedentariness (accelerometer and daily diary)

Figure 16: Frequency ROC curve analysis for Week 2 moderate-to-vigorous-intensity (accelerometer and daily diary)

Figure 17: Frequency ROC curve analysis for Week 2 mild-intensity (accelerometer and daily diary) 108

Figure 18: Frequency ROC curve analysis for Week 2 sedentariness (accelerometer and daily diary) 109

Figure 19: Frequency ROC curve analysis for Week 3 moderate-to-vigorous-intensity (accelerometer and daily diary)

Figure 20: Frequency ROC curve analysis for Week 3 mild-intensity (accelerometer and daily diary)

Figure 21: Frequency ROC curve analysis for Week 3 sedentariness (accelerometer and daily diary)

Figure 22: Duration ROC curve analysis for Week 2 moderate-to-vigorous-intensity (accelerometer and GLTEQ)

Figure 23: Duration ROC curve analysis for Week 2 moderate-to-vigorous-intensity (accelerometer and daily diary) 
Figure 24: Duration ROC curve analysis for Week 2 moderate-to-vigorous-intensity (daily diary and GLTEQ) 115

Figure 25: Duration ROC curve analysis for Week 2 mild-intensity (accelerometer and GLTEQ) 116

Figure 26: Duration ROC curve analysis for Week 2 mild-intensity (accelerometer and daily diary)

Figure 27: Duration ROC curve analysis for Week 2 mild-intensity (daily diary and

GLTEQ) 118

Figure 28: Duration ROC curve analysis for Week 2 sedentariness (accelerometer and daily diary)

Figure 29: Duration ROC curve analysis for Week 3 moderate-to-vigorous-intensity (accelerometer and GLTEQ)

Figure 30: Duration ROC curve analysis for Week 3 moderate-to-vigorous-intensity (accelerometer and daily diary)

Figure 31: Duration ROC curve analysis for Week 3 moderate-to-vigorous-intensity (daily diary and GLTEQ) 122

Figure 32: Duration ROC curve analysis for Week 3 mild-intensity (accelerometer and GLTEQ) 123

Figure 33: Duration ROC curve analysis for Week 3 mild-intensity (accelerometer and daily diary) 124

Figure 34: Duration ROC curve analysis for Week 3 mild-intensity (daily diary and GLTEQ) 125

Figure 35: Duration ROC curve analysis for Week 3 sedentariness (accelerometer and 


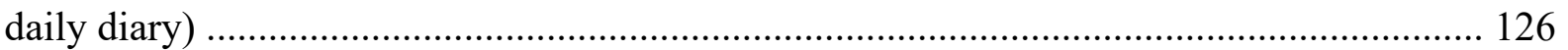


Comparing Measures of Physical Activity Intensity, Duration, and Frequency Using Receiver Operator Characteristic Curve Analyses

\section{Introduction}

Physical activity, or skeletal muscle-induced bodily movement that requires energy expenditure (World Health Organization [WHO], 2017a), is associated with many psychological and physical health benefits. These benefits include increased wellbeing and quality of life (Barwais, Cuddihy, \& Tomson, 2013; Davies, Vandelanotte, Duncan, \& van Uffelen, 2012), decreased anxiety and depression (Brunes, Augestad, \& Gudmundsdottir, 2013; de Mello, de Aquino Lemos, Antunes, Bittencourt, Santos-Silva, \& Tufik, 2013), a decreased risk of developing chronic health conditions (Lee et al., 2012), a longer life expectancy (Lee et al., 2012; Moore et al., 2012), and an increase in physical functioning, including fitness (Chou, Hwang, \& Wu, 2012; Santos et al., 2012). Along with the benefits related to psychological and physical health, there are also connections between physical activity and other components of functioning, including cognition. Research shows that physical activity is associated with the preservation of or an increase in some cognitive skills later in life (Langlois et al., 2013; Zhu et al., 2016) and a decreased risk of developing dementia (Buchman et al., 2012; Verdelho et al., 2012). Given these benefits, it is important to encourage adults to maintain a consistent physical activity routine and to be knowledgeable of the three important physical activity components: intensity, duration, and frequency.

To accurately measure physical activity, intensity, duration, and frequency must be all considered (e.g., Department of Health and Human Services [HHS], 2008; Schoenborn, Adams, \& Peregoy, 2013). However, because it is a multifaceted behavior (Butte, Ekelund, \& Westerterp, 2012; Gabriel, Morrow, \& Woolsey, 2012), measuring and reporting physical 
activity can be complicated.

\section{Physical Activity Recommendations}

Participating in physical activity is beneficial for people of all ages. Adults are encouraged to engage in 150 minutes of moderate-intensity or 75 minutes of vigorous-intensity physical activity each week (HHS, 2008; Schoenborn et al., 2013; World Health Organization [WHO], 2019). Additionally, it is recommended that adults take part in various strength-training exercises two days each week (HHS, 2008; WHO, 2019). Along with these guidelines, there are additional recommendations for older adults. If older adults are not able to engage in 150 minutes of moderate- or 75 minutes of vigorous-intensity weekly physical activity, it is recommended that they engage in as much physical activity as they can safely complete (HHS, 2008; WHO, 2019). Although the guidelines are available to the public, only $20 \%$ of American adults engage in the recommended amount of aerobic physical activity and strength-training exercises (HHS, 2019; Schoenborn et al., 2013). For the future, not only is it important to increase physical activity across adults, but to also provide them with information about the three physical activity components: intensity, frequency, and duration.

\section{Physical Activity Intensity}

Intensity refers to the strenuousness and effort required to complete the activity (Center for Disease Control and Prevention [CDC], 2015a). There are four physical activity intensities: vigorous, moderate, mild, and sedentary. Vigorous is the most intense form of physical activity, moderate as an average intensity, mild represents a low level of physical activity, and sedentary corresponds to inactivity (CDC, 2015a; HHS, 2008). Vigorous-intensity physical activity may include running, fast cycling, or competitively engaging in sports (WHO, 2017b). Examples of moderate-intensity physical activity can include brisk walking, dancing, or hiking (WHO, 
2017b). Mild-intensity physical activities could include leisure walking or light household chores, and examples of sedentariness include sitting to watch television or use the computer (HHS, 2017). Intensity relates to the rate of energy expenditure, meaning activity intensities will vary across people of different weight and fitness ability (WHO, 2017b). These differences in intensity become visible upon measuring the metabolic equivalents (METs) for various people engaging in the same activity.

Analyzing METS or the ratio between the working and resting metabolic state (WHO, 2017b), is an accepted method to assess physical activity intensity (e.g., HHS, 2008; Strath et al., 2013). Although METs vary across people based on weight, age, and sex (e.g., HHS, 2008), METs for each intensity level have been documented by studying common activities in laboratory settings (Staudenmayer, Pober, Crouter, Bassett, \& Freedson, 2009). Vigorous physical activity intensity is any activity that requires more than 6.0 METs and burns more than 7 kilocalories each minute (WHO, 2017b). Moderate physical activity ranges in MET values from 3.0 to 6.0, with one burning between 3.5 and 7 kilocalories each minute (WHO, 2017b). A mild-intensity physical activity scores between 1.1 to 2.9 METs, and a sedentary behavior is designated as anything under 1.1 METs (HHS, 2008). Although calculated METs is common, there are also other methods of studying physical activity intensity.

Assessing heart rate is a validated method to measure physical activity intensity, as heart rate is associated with energy expenditure (Butte et al., 2012; Watkinson et al., 2010). This methodology entails estimating blood flow through wrist sensors (Dooley, Golaszewski, \& Bartholomew, 2017; Intel Corporation, 2015). These ranges can be calculated by subtracting one's age from 220 and then multiplying this score by $0.50-0.70$ for moderate-intensity physical activity or by $0.70-0.85$ for vigorous-intensity physical activity (CDC, 2015b). These ranges are 
important to consider since engaging in moderate- and vigorous-intensity physical activity is ideal (e.g., HHS, 2008; Moore et al., 2012; Nelson et al., 2007; Schoenborn et al., 2013).

Higher physical activity intensities are associated with more positive outcomes, in comparison to lower intensities (Netz, Wu, Becker, \& Tenenbaum, 2005; Wen et al., 2011; Haskell et al., 2007; Powell, Paluch, \& Blair, 2011). For example, although mild-intensity physical activity can be successful at reducing the risk of mortality in comparison to total sedentariness, a higher intensity is associated with an even lower mortality rate (Wen et al., 2011). Additionally, those who live a sedentary lifestyle experience less of the psychological (e.g., Netz et al., 2005) and physical benefits of life (e.g., Warburton, Nicol, \& Bredin., 2006;

Wen et al., 2011) than someone who engages in a higher intensity of physical activity (Powell et al., 2011). Even if one engages in vigorous-intensity physical activity, though, it is important to study the length, or duration, of the physical activity, as benefits accrue over an increasing amount of time.

\section{Physical Activity Duration}

Physical activity duration refers to the amount of time spent engaging in a physical activity behavior (HHS, 2008). Although guidelines exist for physical activity duration (e.g., HHS, 2008; Schoenborn et al., 2013), it is not as clearly addressed in the current literature (Haskell et al., 2007). However, results generally indicate that as physical activity duration increases, positive health outcomes, such as a decreased risk of developing chronic conditions (Haskell et al., 2007), also increase (Chodzko-Zajko et al., 2009).

Various methods can be utilized to assess duration. Accelerometers (e.g., Freedson, Bowles, Troiano, \& Haskell, 2012; Ortlieb et al., 2014) or a stopwatch can track minutes and hours of activity (e.g., Wen et al., 2011). Accelerometers, however, may provide a more accurate 
report of duration over self-report methods because recall is often inaccurate (Hallal et al., 2012; Sirard, Hannan, Cutler, Graham, \& Neumark-Sztainer, 2013).

Other biases may also affect the accuracy of self-reported duration. Adams and colleagues (2005) concluded that social desirability contributes to inaccurate self-reported accounts of duration for mild- and moderate-intensity physical activity. Additionally, some questionnaires may not assess physical activity that is under a certain duration (Tudor-Locke \& Myers, 2012; Prince et al., 2008), such as the Godin Leisure-Time Exercise Questionnaire (GLTEQ; Godin \& Shephard, 1997). The GLTEQ tries to decrease the recall burden by having participants recall physical activity duration in increments of 15 minutes. These issues are specific to subjective data and do not extend to objective data. Objective measures of physical activity are additionally helpful because many automatically track duration, along with intensity and frequency of physical activity.

\section{Physical Activity Frequency}

Frequency refers to how often one engages in physical activity (e.g., Haskell et al., 2007; HHS, 2008; Netz et al., 2005), such as being physically active five times per week. The guidelines suggest at least five days of physical activity engagement for optimum health (Haskell et al., 2007). Generally, increased frequency is associated with greater health outcomes, as well (Chodzko-Zajko et al., 2009; Haskell et al., 2007). McDonnell and colleagues (2013) concluded from hazards ratios (HR) that middle-aged and older adult men who were active four times per week were less likely to experience a stroke than one who engaged in physical activity less frequently $(\mathrm{HR}=1.30)$.

Frequency can be assessed by reporting the number of days (Haskell et al., 2007) or sessions of physical activity (McDonnell et al., 2013), using a questionnaire or logged by an 
accelerometer. Accurately assessing frequency is one benefit of using accelerometers (e.g., Sylvia, Bernstein, Hubbard, Keating, \& Anderson, 2014), as participants may be prone to give incorrect retrospective reports due to problems with recall (Hallal et al., 2012).

\section{Subjective Measures of Physical Activity}

Subjective measures include questionnaires, daily diaries, and other self-report methods (Sylvia et al., 2014; Warren et al., 2010). Self-reports are the most commonly used physical activity measure (Castillo-Retamal \& Hinckson, 2011; Sylvia et al., 2014; Warren et al., 2010). There are benefits to using subjective measures, including the ability to sample a large number of participants and the cheap cost (Sallis \& Saelens, 2000; Sylvia et al., 2014; Warren et al., 2010). Limitations, however, include potentially inaccurate reports for intensity, duration, and frequency of physical activity; the influence of potential biases; culture specificity; and high cognitive demands (Sallis \& Saelens, 2000; Sylvia et al., 2014; Warren et al., 2010). Specific questionnaires have been created to assess physical activity among different age groups, such as the Physical Activity Survey for the Elderly (e.g., PASE; Washburn, Smith, Jette, \& Janney, 1993). Not all studies utilize age-specific measures, but instead opt to use general physical activity measures. Daily diary accounts, however, allow for a more comprehensive analysis of the behavior (Gotsis et al., 2013; Hektner, Schmidt, \& Csikszentmihalyi, 2007; Nehrkorn-Bailey, Reardon, \& Patrick, 2018). Studies that utilize daily diary accounts have a plethora of data, as responses are collected multiple times a day; improved ecological validity; and a reduced impact of recall and other biases common among other subjective measures (Hektner et al., 2007; Nehrkorn-Bailey et al., 2018; Wichers et al., 2011). With daily diary data, physical activity can be assessed close to the time of engagement. Although there are benefits to using subjective measures, objective measures may offer a cleaner assessment of physical activity components. 


\section{Objective Measures of Physical Activity}

Objective measures include accelerometers, pedometers, (in)direct calorimetry, doublylabeled water (DLW), oxygen consumption $\left(\mathrm{VO}_{2}\right)$, and direct observation (Sylvia et al., 2014; Prince et al., 2008). These methodologies are objective as there is a direct link to one's physical activity, which is being measured in real time (Sylvia et al., 2014; Warren et al., 2010). Although pedometers track number of steps (Kinnunen et al., 2011), accelerometers can track number of steps; calories burned; heart rate; and intensity, duration, and frequency of physical activity (Hutto et al., 2013; Intel Corporation, 2015; Kelly et al., 2013; Motl, McAuley, \& Dlugonski, 2012). Indirect calorimetry measures the amount of oxygen consumed and the amount of carbon dioxide produced; however, direct calorimetry measures the body's heat production (Oshima et al., 2016). For the doubly labeled water techniques, participants are required to drink water with isotopes at baseline. After 7-12 days, participants come back to laboratory to have the isotope levels re-evaluated (Oshima et al., 2016). Oxygen consumption $\left(\mathrm{VO}_{2}\right)$ measures heart rate, oxygen, and carbon dioxide at rest and then during an exercise task (Bichay et al., 2016). The (in)direct calorimetry, doubly labeled water, and oxygen consumption techniques require advanced equipment, which is not be feasible for the general public to use for tracking their physical activity. Instead, accelerometers are often used as the standard for comparison with other physical activity measures (Lee, Macfarlane, Lam, \& Stewart, 2011). Accelerometers can provide people with a reliable, validated, and more cost-efficient way of analyzing physical activity on a daily basis (Hutto et al., 2013; Kelly et al., 2013; Kowalski, Rhodes, Naylor, Tuokko, \& MacDonald, 2012; Strath et al., 2013; Sylvia et al., 2014). Many of these tracing devices can also assess a variety of other behaviors, including sleep (Montgomery-Downs, Insana, \& Bond, 2012). However, accelerometers have been found to overestimate sleep duration 
(Meltzer, Hiruma, Avis, Montgomery-Downs, \& Valentin, 2015). High cost, incomplete data, and advanced statistical analyses are drawbacks of objective methodologies (Warren et al., 2010).

\section{Validity of Physical Activity Measures}

To assess agreement across methodologies, physical activity can be measured using both subjective and objective measures within a study. However, findings across studies vary, leaving inconsistencies. The statistical analyses utilized for these studies, however, have remained consistent. Spearman rank order correlation coefficients and Bland-Altman plots are often utilized to test agreement across physical activity measures (e.g., Dyrstad et al., 2014; Stahl \& Insana, 2014; Sternfeld et al., 2011). In the following sections, Cohen's criteria for effect sizes (Cohen, 1988) were used to assess associations (weak, medium, or strong) across methodologies, as has been done in another study (e.g., Boon, Hamlin, Steel, \& Ross, 2010). Effect sizes ranging from 0.1-0.29 represents a week association, 0.3-0.49 represents a medium association, and 0.5 and above indicates a strong association (Cohen, 1988). Assessing effect sizes is an important method of evaluating relationships across variables. See Table 1 for the validation literature's findings on intensity, frequency, and duration of physical activity across subjective and objective measures.

Intensity: Validation with Questionnaires and Accelerometers. One concern with subjective methodologies is that participants may be incorrectly labeling their physical activity intensity (e.g., Troiano et al., 2007). This can be assessed, however, with the inclusion of an objective measure. Stahl and Insana (2014) concluded 70\% of participants' accelerometerderived kilocalorie data was within one standard deviation of the mean difference between the kilocalories noted in a questionnaire, with $100 \%$ of the data falling within two standard 
deviations of the mean difference. Previous validation studies that incorporated multiple measures have focused more on duration and less on intensity (e.g., Dyrstad, Hansen, Holme, \& Anderssen, 2014; Hagströmer, Ainsworth, Oja, \& Sjöström, 2010), resulting in a lack of intensity-specific data.

Although data are sparse, consistent medium-sized associations have been documented for vigorous-intensity physical activity. By assessing kilocalories, one study found medium agreement between the International Physical Activity Questionnaire (IPAQ; Craig et al., 2003) and Computer Science and Applications Inc. (CSA; Computer Science and Applications, Inc., 1997) accelerometer for vigorous-intensity physical activity ( $\rho=.45$; Vandelanotte, de Bourdeaudhuij, Philippaerts, Sjostrom, \& Sallis, 2005). Studying caloric expenditure among 870 older adults, Hekler and colleagues (2012) found medium agreement for moderate-to-vigorous physical activity between the Community Health Activities Model Program for Seniors (CHAMPS; Stewart et al., 2000) and Actigraph (Actigraph, Manufacturing Technology Inc.) accelerometer data $(\rho=.40)$. Thus, adults are fairly accurate at reporting vigorous-intensity physical activity (Hallal et al., 2012; Hekler et al., 2012; Strath et al., 2013).

There is a lack of data on the accuracy of labeling moderate- and mild-intensity physical activity. Comparing accelerometer and questionnaire data in a chi-square analysis, across 3,975 older participants from a 9-day protocol, Sabia and colleagues (2014) found approximately $47 \%$ of participants were correctly classified as engaging in vigorous-intensity physical activity, $36 \%$ were correctly classified as engaging in moderate-intensity physical activity, and $48 \%$ were correctly classified as engaging in mild-intensity physical activity. This cross-classification across accelerometer and questionnaire data was considered low by the researchers, with $32.1 \%$ of participants overestimating mild-intensity physical activity as moderate-intensity physical 
activity, 30.4\% underestimating and 33.4\% overestimating moderate-intensity physical activity, and $31.9 \%$ of participants mis-labeling vigorous physical activity for moderate-intensity physical activity (Sabia et al., 2014) Additionally, Vandelanotte and colleagues (2005) found no significant relation across methodologies for moderate- or mild-intensity physical activities when analyzing kilocalories. These low numbers suggest significant inaccuracy in labeling mild- and moderate- intensities of physical activity.

Along with inaccurate reports of mild- and moderate-intensity physical activity, the relationship across measures for sedentary behaviors is unclear. A weak relation has been documented between self-reported sedentariness and that measured by accelerometers (KozeyKeadle, Libertine, Lyden, Staudenmayer, \& Freedson, 2010). For example, Watkinson and colleagues (2010) assessed physical activity awareness among sedentary adults. The researchers concluded that among 365 younger and middle-aged adults, just under one-third of participants incorrectly labeled themselves as being active, when they were actually sedentary, based on heart-rate monitoring (Watkinson et al., 2010). Kozey-Keadly and colleagues (2010) examined various counts per minute, which are used to label intensity, finding there to be a crucial cut-off to accurately assess sedentariness. In one study, the accelerometer was able to differentiate between sedentary behavior and activity, but another could not differentiate between sedentariness and activity (Kozey-Keadly et al., 2010). However, measuring sedentariness may be difficult as there is not a standardized definition for this level (Gibbs, Hergenroeder, Ktzmarzyk, Lee, \& Jakicic, 2015).

Intensity: Validation with Daily Diaries. Validation studies have not often included daily diaries. However, Stel and colleagues (2004) utilized daily diaries, pedometers, and the LASA Physical Activity Questionnaire (LAPAQ) to assess the validity of physical activity data 
from 439 older adults. When participants were grouped by intensity, $60 \%$ of participants correctly assessed their intensity in the LAPAQ, 19\% over-estimated their intensity, and 21\% under-estimated their intensity, in comparison to the daily diary data (Stel et al., 2004).

Furthermore, there was medium agreement between the pedometer and daily diary $(\rho=.43$; Stel et al., 2004). It is important to note that Sternfeld and colleagues (2011) concluded from a pilot study that in comparison to traditional pen-and-paper daily diaries, electronic daily diaries included more complete intensity information.

Duration: Validation with Questionnaires and Accelerometers. An additional concern may be that self-reported data or accelerometer data are not measuring the full duration of physical activity engagement (e.g., Troiano et al., 2007). Only a weak-to-medium level of agreement has been documented across methodologies for duration of total physical activity (Ekelund et al., 2006; Hagströmer, Oja, \& Sjöström, 2006). So, although duration is more often analyzed than the other components (e.g., Dyrstad et al., 2014; Hagströmer et al., 2010), there could be measurement problems across methodologies, affecting duration assessment.

Duration of vigorous physical activity duration measured across methodologies demonstrates consistent positive associations (e.g., Bull, Maslin, \& Armstrong, 2009; Hagströmer et al., 2006), but the following studies show low-to-poor agreement across studies. Comparing Actigraph accelerometer and IPAQ data among 1,751 younger, middle-aged, and older adults, duration of vigorous physical activity in adults was associated at a weak-to-medium level for some younger and middle-aged adults $(\rho=.25-.47$; Dyrstad et al., 2014). There is variability, as one study found a weak level of agreement ( $\rho=.23-.26$; Bull et al., 2009) and others demonstrated medium-to-strong agreement across methodologies for duration of vigorous 
physical activity $(\rho=.39-.63$; Hagströmer et al., 2006; Herrmann, Heumann, Ananian, \& Ainsworth, 2012; Vandelanotte et al., 2005). Other research shows participants over-reported their duration of vigorous physical activity (Dyrstad et al., 2014; Hagströmer et al., 2010). Based on findings, adults are at least moderately accurate at reporting their duration of vigorous physical activity.

Labeling the duration of moderate-to-vigorous physical activity in various contexts may be more challenging, leading to inconsistencies ranging from insignificant results to mediumstrength associations. A weak relation was documented between questionnaire and accelerometer data for duration of moderate-to-vigorous physical activity $(\rho=26 ; r=.17$; Ekelund et al., 2006; Herrmann et al., 2012), with other studies finding a medium relation ( $\rho=.36-.40$; Hagströmer et al., 2006; Hekler et al., 2012; van Holle, de Bourdeaudhuij, Deforche, van Cauwenberg, \& van Dyck, 2015; Vandelanotte et al., 2005). However, assessing duration of physical activity in different contexts, such as at home or work, may be especially difficult. Hagströmer and colleagues (2010) found that 980 younger, middle-aged, and older adults were only marginally accurate at reporting their duration of moderate-to-vigorous physical activity using the IPAQ, while at work $(\rho=.15)$, while commuting $(\rho=.08)$, and in their leisure time $(\rho=.26)$, when compared to Actigraph accelerometer data. Adults were not successful at labeling their duration of moderate-to-vigorous physical activity while engaging in household activities $(\rho=-.02$; Hagströmer et al., 2010). Assessing duration of moderate-to-vigorous physical activity appears to be more difficult than assessing vigorous physical activity duration.

Duration of moderate physical activity data shows inconsistent relations ranging from non-significant to medium-strength agreement across methodologies. Hagströmer and colleagues (2006) concluded self-reported duration of moderate physical activity data from the IPAQ among 
46 healthy younger and middle-aged adults were not significantly related to Actigraph accelerometer data, replicating other work (e.g., Bull et al., 2009; Vandelanotte et al., 2005). However, Dyrstad and colleagues (2014) found a significant medium relation between the IPAQ and ActiGraph accelerometer only for older adults $(\rho=.40)$ and found that all younger, middleaged, and older adults under-reported their duration of moderate physical activity. Slootmaker, Schuit, Chinapaw, Seidell, and van Mechelen (2009) also found a medium relation between the Activity Questionnaire for Adolescents \& Adults (AQuAA; Chinapaw, Slootmaker, Schuit, van Zuidam, \& van Mechelen, 2009) and PAM (PAM B.V.) accelerator for duration of moderate physical activity, but with younger and middle-aged adults over-reporting. This medium-level agreement has been documented elsewhere $(\rho=.36$, Herrmann et al., 2012), along with a weak relation documented between methodologies, too $(\rho=.23-.27$; Bull et al., 2009; Hagströmer et al., 2010). Agreement for duration of moderate physical activity across methodologies varies from nonsignificant to a medium-level relation.

Less literature focuses on duration of mild physical activity. However, Hekler and colleagues (2012) found a weak level of agreement across methodologies for duration of mild physical activity $(\rho=.27)$, but only when it was a more intense mild activity. On the contrary, Vandelanotte and colleagues (2005) did not find a significant association across methodologies for duration of mild physical activity. Sabia and colleagues (2014) assessed physical activity duration in various contexts, finding participants were largely inaccurate at labeling their duration of mild physical activity while completing housework $(\rho=.09)$, but were slightly more accurate at labeling duration when walking $(\rho=.21)$. Again, the context may impact duration assessment, although adults may be only slightly accurate at labeling duration of mild physical activity. 
Adults are often inaccurate when noting the amount of time they spend engaging in sedentary activities. Some of the previous literature highlights a non-significant relation across subjective and objective reports (Bull et al., 2009; Hagströmer et al., 2006; Herrmann et al., 2012), although other studies have documented a weak-to-medium level of agreement for duration of sedentary behavior $(\rho=.12-.46 ; r=.16$; Bull et al., 2009; Ekelund et al., 2006; Hekler et al., 2012) at least among younger and middle-aged adults (Dyrstad et al., 2014). This weak-to-medium relation details that participants tend to under-report their duration of sedentary behaviors (Dyrstad et al., 2014), with this conclusion documented in another study, too (KozeyKeadle et al., 2010). Other research indicates that participants tend to over-estimate their duration in sedentary behaviors (Hagströmer et al., 2010). Findings on sedentary behavior duration are inconsistent, with a range of agreement documented.

Duration: Validation with Daily Diaries and Questionnaires. Daily diary methodologies are not frequently utilized to assess intensity or duration in the validation literature (Gotsis, Wang, Spruijt-Metz, Jordan-Marsh, \& Valente, 2013; Sallis \& Saelens, 2000). However, studies that have incorporated such diaries have found a strong level of agreement across weekly subjective methodologies (i.e., questionnaire, other diary report) for total duration of activity ( $\rho=.61-.68$; Hagströmer et al., 2006; Stel et al., 2004; Sternfeld et al., 2011). Sternfeld and colleagues (2011) conducted a validation study to assess electronic daily diary data against pen-and-paper daily diary data from 623 middle-aged adults. From the validation study, a strong relation was found for duration of moderate-to-vigorous physical activity when comparing the two types of daily diaries $(\rho=.79$; Sternfeld et al., 2011). Vandelanotte and colleagues (2005) also found a high level of agreement for duration of vigorous physical activity $(\rho=.79)$ and a medium-strength relation for duration of moderate physical activity $(\rho=.32)$ when 
comparing daily diary and questionnaire data. For sedentary duration, Hagströmer and colleagues (2005) found a high level of agreement between daily diary and questionnaire data, as well $(\rho=.75)$.

Duration: Validation with Daily Diaries and Accelerometers. Analyzing data from the electronic daily diary and accelerometer, there was medium agreement for duration of vigorous physical activity $(\rho=.31-.35)$, weak agreement for of duration moderate physical activity $(\rho=$ .27-.28), and a lack of agreement for duration of mild physical activity and sedentary behaviors (Sternfeld et al., 2011). Daily diary data appear to be more strongly related to other subjective data rather than objective data.

Frequency: Validation with Questionnaires and Accelerometers. Although many of the methodologies utilized in the previous literature (i.e., questionnaire, accelerometer) can measure frequency, validation studies often do not report this component (e.g., Taylor-Piliae et al., 2010). One study that did assess frequency, albeit, with a more significant focus on duration, found questionnaire responses significantly over-estimated frequency, in comparison to accelerometer data (Boon et al., 2010).

One reason frequency is often not examined in studies is because of the way physical activity guidelines are discussed, which succinctly combine intensity-, duration-, and frequencyspecific information. Thus, some studies combine intensity, duration, and frequency in to a single metric. Tucker, Welk, and Beyler (2011) assessed self-reported physical activity and Actigraph accelerometer data in comparison to national guidelines among 3,082 younger, middle-aged, and older adults. Duration was multiplied with frequency, resulting in a minutesper-week variable. Participants over-estimated their moderate-to-vigorous physical activity duration*frequency, with a weak association for moderate-to-vigorous duration*frequency $(\rho=$ 
$.27)$, a weak association for leisure moderate-to-vigorous physical activity $(\rho=.29)$, and a weak association for household moderate-to-vigorous physical activity $(\rho=.08$; Tucker et al., 2011). This combined variable was then labeled "physical activity level" (Tucker et al., 2011). Other studies have also combined the duration and intensity variables into a single unit (e.g., Hoos, Espinoza, Marshall, \& Arredondo, 2012). For example, Hoos and colleagues (2012) found medium-to-strong agreement for vigorous physical activity duration*frequency $(\rho=.42-.51)$, a medium-strength relation for moderate-to-vigorous physical activity duration*frequency ( $\rho=$ $.38)$, and medium agreement for mild physical activity duration*frequency $(\rho=.33)$ across the Global Physical Activity Questionnaire (GPAQ; Bull et al., 2009) and Actigraph accelerometer data. Although the minutes-per-week variable combines duration and frequency, other research considered minutes per week a measure of duration (e.g., Hupin et al., 2015). Thus, there is a lack of clarity and overlap in duration and frequency measurement. Since the guidelines do contain frequency-related information (e.g., HHS, 2008), it is crucial to also validate physical activity frequency.

\section{Individual Differences in Accurately Reporting Physical Activity}

Along with understanding some of the gaps in the current physical activity literature, it is also important to understand how certain groups of people report physical activity intensity, frequency, and duration. Representative of development across the life span, change occurs over time, with the potential for development to be complex (Baltes, Lindenberger, \& Staudinger, 2006; Schaie, 2010). Successful development focuses on the maximization of gains and minimization of losses (Freund, Nitkitin, \& Riediger, 2012), such that adults can engage in physical activity even if they experience chronic health conditions or functional disabilities. Considering the benefits, physical activity is a positive behavior for successful development 
across groups. Sex, age, and intrinsic motivation are some variables that may be associated with group differences in accuracy of reporting on the physical activity components.

Sex. The current literature has not extensively studied differences in reporting accuracy across males and females. Across methodologies, females tend to over-report their physical activity intensity (Prince et al., 2008). Findings for duration, however, are inconsistent. One study found that men are more likely to over-report their physical activity duration (Watkinson et al., 2010). However, in mixed studies, males over-reported their duration of vigorous-intensity physical activity but under-reported their durations of moderate-intensity physical activity and sedentariness (Dyrland et al., 2013). Cleland and colleagues (2014) found females overestimated their duration of moderate-to-vigorous-intensity physical activity in comparison to accelerometer data. There are not documented sex differences for frequency (Duncan, Hall, Wilson, \& Jenny, 2010). In addition to sex differences, age also appears to be an important variable to assess differences in physical activity components.

Age. There are noted age differences for reporting accuracy in duration and intensity. Slootmaker and colleagues (2009) concluded adults were likely to over-report duration of moderate-, vigorous-, and moderate-to-vigorous-intensity physical activity, but Tucker and colleagues (2011) concluded that only adults aged 70 years or higher under-reported their moderate-to-vigorous-intensity physical activity. Dyrstad and colleagues (2014) concluded all adults in their study under-reported their duration of moderate-intensity physical activity and sedentary behavior. For sedentariness, one study found younger and older adults underreported the duration of time spent in a sedentary behavior (Hagströmer et al., 2006). Conversely, Dyrstad and colleagues (2014) found younger and middle-aged adults to be more accurate than older adults aged 65 years and above in reporting their duration in a sedentary behavior. 
Motivation to be Physically Active. Motivation, or one's reason to be active (e.g., Hagger \& Chatzisarantis, 2014), may affect physical activity components. Intrinsic motivation implies one engages in physical activity due to interest in the behavior (e.g., Deci \& Ryan, 2002; Teixeira, Carraca, Markland, Silva, \& Ryan, 2012). Conversely, extrinsic motivation entails engaging in physical activity to attain a reward (e.g., Deci \& Ryan, 2002). Some studies have concluded that intrinsic motivation is a significant predictor of physical activity (Lauderdale, Yli-Piipari, Irwin, \& Layne, 2015; Ruffault, Bernier, Juge, \& Fournier, 2016; Teixeira et al., 2012), but with no specification regarding which physical activity components were assessed. Friederichs, Bolman, Oenema, and Lechner (2015) found intrinsically motivated participants to be more likely to engage in a higher duration and frequency of moderate-to-vigorous physical activity compared to participants with low motivation. Extrinsic motivation, however, appears to be negatively associated with physical activity, although others have documented a nonsignificant relation (Teixeira et al., 2012; Vancampfort et al., 2013).

\section{Statement of the Problem}

For public health and as a means to promote healthy aging, there are many recommendations for engaging in physical activity. However, in order for people of all ages to successfully meet the physical activity recommendations, they need to be able to accurately monitor their physical activity intensity, duration, and frequency. In its current state, the field has not fully examined the measures of physical activity, with relatively little attention to intensity, duration, and frequency (e.g., Bull et al., 2009; Dyrland et al., 2013; Hekler et al., 2012; Watkinson et al., 2010). Many studies merely discuss "physical activity" without information regarding intensity, duration, or frequency (e.g., Bull et al., 2009; Hekler et al., 2012; Watkinson et al., 2010). Examining multiple aspects of physical activity is important because the 
recommendations include specific details regarding intensity, frequency, and duration.

Moreover, there has been relatively little empirical attention examining associations among various objective measures and subjective reports of physical activity. This is important to address because the general public needs effective equipment to assess their daily physical activity (e.g., Hagströmer et al., 2006).

\section{The Current Study}

This study expands and adds to the current literature on measuring physical activity by triangulating physical activity intensity, duration, and frequency assessments across daily diary (ESM), questionnaire, and accelerometer data. Currently, there is a lack of data on physical activity intensity and frequency, lack of clarity on which physical activity component is being analyzed within studies, and lack of literature on the agreement across various subjective and objective measures. By addressing multiple gaps in the literature and conducting advanced statistical analyses, which have not been previously utilized to study this topic, this study significantly adds to the current physical activity literature.

\section{Research Questions and Hypotheses}

\section{Research Question 1}

Are the subjective measures (GLTEQ and diary report) associated with the objective measure (accelerometer)?

Hypothesis 1a. It was hypothesized that reports from the GLTEQ and daily diaries would over-report physical activity intensity (moderate-to-vigorous, mild, and sedentary; e.g., Sternfeld et al., 2011; Watkinson et al., 2010), duration (e.g., Hagströmer et al., 2010; Slootmaker et al., 2009), and frequency (e.g., Boon et al., 2010) in comparison to data gathered from the accelerometer. 
Hypothesis 1b. Based on the findings from Hagströmer and colleagues (2006) and Sternfeld and colleagues (2011), it was hypothesized that the daily diary method, in comparison to the GLTEQ, would exhibit the strongest agreement with the accelerometer data, regarding reporting physical activity intensity (moderate-to-vigorous and mild) and duration.

Hypothesis 1c. It was hypothesized that the relation between the GLTEQ and the accelerometer would be weak $(r<.30)$ for mild intensity (e.g., Hekler et al., 2012; Sabia et al., 2014; Watkinson et al., 2010) and duration data (e.g., Hagströmer et al., 2010; Hekler et al., 2012). The relation between the GLETQ and accelerometer would be weak-to-medium $(.10<r<$ .49) for moderate-to-vigorous physical activity intensity and duration (e.g., Hekler et al., 2012; Vandelanotte et al., 2005).

Hypothesis 1d. Based on the findings from Sternfeld and colleagues (2011) and Vandelanotte and colleagues (2005), the hypothesized relation between the daily diary reports and the accelerometer would be weak-to-medium $(.10<r<.49)$ for intensity (moderate-tovigorous, mild, and sedentary), duration, and frequency.

Hypothesis 1e. The association between the GLTEQ and daily diary report was hypothesized to be medium-to-strong $(.29<r<1.00)$ for intensity (moderate-to-vigorous and mild) and duration, which is based on the findings from the previous literature (Hagströmer et al., 2006; Latham et al., 2008; Sternfeld et al., 2011).

\section{Research Question 2}

Does age influence agreement for intensity, duration, and frequency across the accelerometer, daily diary, and GLTEQ?

Hypothesis 2a. Based on the inconsistent findings regarding age (e.g., Dyrstad et al., 2014; Slootmaker et al., 2009), it was hypothesized that when comparing data across age groups, 
the accelerometer, daily diary, and GLTEQ data would be associated similarly across younger adults (Group 1) and middle-aged and older adults (Group 2) for intensity (moderate-tovigorous, mild, and sedentary), duration, and frequency.

\section{Research Question 3}

Does sex influence agreement for intensity, duration, and frequency across the accelerometer, daily diary, and GLTEQ?

Hypothesis 3a. Based on the largely inconsistent findings for sex (e.g., Cleland et al., 2014; Duncan et al., 2010; Dyrstad et al., 2014), it was hypothesized that the relations for the accelerometer, daily diary, and GLTEQ data would be associated similarly across males and females for intensity (moderate-to-vigorous, mild, and sedentary), duration, and frequency.

\section{Research Question 4}

Does intrinsic motivation to be physically active influence agreement for intensity, duration, and frequency across the accelerometer, daily diary, and GLTEQ?

Hypothesis 4a. It was hypothesized that for intensity (moderate-to-vigorous, mild, and sedentary), duration, and frequency, the associations between the accelerometer, daily diary, and GLTEQ would demonstrate a medium level of agreement $(.29<\mathrm{r}<.49)$ when participants are more highly motivated to be physically active. Based on research, intrinsic motivation is associated with more moderate-to-vigorous physical activity (Friederichs et al., 2015); whereas a lack of motivation has been associated with physical activity decline (Teixeira et al., 2012).

\section{Method}

\section{Procedure}

Data were provided by 62 adults who participated in the Age and Behavioral Coaching across Domains study (ABCD; Patrick \& Gentzler, PIs, 2014). The parent study included a 12- 
week protocol to assess health along a variety of domains, including emotion, cognition, social relationships, religion, psychological, and physical health. Participants were recruited from community events and by distributing flyers across West Virginia University and the larger community. Before being enrolled into the study, participants first completed a prescreen questionnaire $(\mathrm{N}=1,445)$. Participants who were 18 years old or older, currently inactive or engaging in infrequent physical activity, noted a physical activity goal on the prescreen and expressed the desire to work on that goal, and provided a valid e-mail address were then invited into the study. A total of 154 people provided their e-mail address, but based on ineligibility and participant recruitment to an affiliated study, only 62 participants were invited to the current study. Six participants quickly withdrew from the study, leading to a total sample size of 56 participants for the current study. See Appendix A for prescreen information. During the first twenty-one days of the study, participants were given accelerometers to wear and attended inperson meetings once a week, totaling four visits to the lab. Additionally, participants completed four daily diary assessments each day for the twenty-one days. Each daily diary assessment consisted of e-mailed questions sent to participants, which took fewer than two minutes, on average, to complete. At the last in-person meeting, which took place at the end of the twentyone days, participants returned the accelerometer. At weeks 4, 8, and 12, all of which took place after the in-person meetings had concluded, participants received online questionnaires, which assessed their physical activity and other health-related components. Participants were paid $\$ 50$ for their participation in the in-person meetings, and up to an additional $\$ 50$ for their completion of the online surveys at weeks 4,8 , and 12 . Information related to the full study can be found in Figure 1.

The current study utilized data from weeks 2 and 3 . Week 1 was treated as a practice 
week for participants because they were still learning to continuously wear the accelerometer and answer the four daily diary e-mails. By weeks 2 and 3, participants were in a more consistent habit of wearing the accelerometer and answering the daily diary e-mails.

\section{Participants}

Fifty-six adults $($ Mage $=43.2, S D=16.5)$ ranging in age from $18-72$ years provided data for the current study. Each participant completed a 1-week recall of physical activity (i.e., GLTEQ), daily diary report (i.e., ESM), and a real-time continuous report of physical activity (i.e., MyBasis accelerometer). The majority were female (76.8\%). Regarding race, $84.7 \%$ of participants identified as Caucasian, 5.1\% African American, and 3.4\% Native Hawaiian, Pacific Islander or Asian. Most (88.1\%) were non-Hispanic in ethnicity. Thirty-nine percent of participants were married, $28.8 \%$ were single and never married, $22 \%$ were divorced, and $1.7 \%$ were widowed. Sixty-nine percent of adults worked full-time, 3.6\% were employed part-time, $7.3 \%$ were retired, and $20 \%$ were students.

\section{Measures}

Demographics. Participants indicated their age, marital status, ethnicity, sex, and highest level of education in the pre-screen. These data were confirmed at the first in-person meeting.

Motivation. Four items from the Goal-Striving Reasons Index (GSRI; Ehrlich, 2012) were used to assess intrinsic motivation at Week 1 (see Appendix B). The GSRI contains 16 items that address various types of motivation, with four of the items specifically addressing intrinsic motivation ("This goal is important to you because you enjoy doing it."). Participants rated how true each statement was for them (ranging from $1=$ Not at all true to $7=$ Very true). A composite score was created based on participants' answers to the four intrinsic items. The current study's intrinsic motivation sub-section of the GSRI $(\mathrm{M}=21.33, \mathrm{SD}=4.78)$ has high 
internal consistency $(\alpha=0.88)$, which has been found in other studies, too $(\alpha=0.90-0.93$; Ehrlich \& Bipp, 2016).

Basis B1 Accelerometer. The Basis B1 accelerometers (Intel Corporation, 2015) provided objective, real-time, continuous accounts of daily physical activity (see Appendix C). Information on intensity (moderate-to-vigorous-intensity, mild-intensity, and sedentary), frequency, and duration of physical activity is recorded. These accelerometers feature five sensors for optical blood flow, perspiration, ambient temperature, body temperature, and 3 axes to measure acceleration (Boletsis, McCallum, \& Landmark, 2015). Based on the moment-tomoment information from the sensors, the wearer's current state is continuously tracked (Boletsis et al., 2015). With these sensors, the accelerometer can monitor heart rate, steps, calories burned, and sleep cycles (Intel Corporation, 2015). The combined moderate-to-vigorousintensity physical activity is often studied in the current literature (e.g., Fanning et al., 2016; Tucker et al., 2011). Thus, moderate and vigorous physical activity data from the other measures were combined to yield indices of moderate-to-vigorous physical activity for intensity-specific activity, duration, and frequency.

The Basis B1 accelerometer is a validated measure for assessing physical activity through heart rate monitoring and energy expenditure (Intel Corporation, 2015). A strong effect $(r=.94)$ was established between the Basis B1 accelerometer and heart rate, as measured by electrocardiography (ECG; Intel Corporation 2015). Strong agreement was also documented between the accelerometer and indirect calorimetry, which assesses energy expenditure (Intel Corporation, 2015). This supports the accelerometer being utilized as the standard, as the ECG and indirect calorimetry are ideal laboratory methodologies (Intel Corporation, 2015). As the 
standard, the accelerometer was dichotomized for all of the current study's analyses. Rules for dichotomizing are discussed in the Results section.

Questionnaire. The Godin Leisure Time Exercise Questionnaire (GLTEQ; Godin \& Shephard, 1997) is a subjective 1-week recall measure of physical activity (see Appendix D). As is common (Motl \& McAuley, 2009), the first part of the GLTEQ was used to assess physical activity intensity and duration. The questions ask how often in the past week, in 15-minute increments, the participant engaged in vigorous-, moderate-, and mild-intensity physical activity. Examples of activities at each intensity are included, providing the participant with ideas of their past week physical activity, as well as ideas for future activity. The moderate-to-vigorousintensity variable was generated by combining the vigorous- and moderate-intensity data. Thus, continuous moderate-to-vigorous- and mild-intensity duration and intensity data from the GLTEQ were analyzed.

Based on past research, the GLTEQ is a reliable and valid measure. A strong effect for test-retest reliability has also been found across two weeks ( $r=.74$; Motl, McAuley, Wynn, Suh, \& Weikert, 2011) and later (Homan \& Tylka, 2014). Additionally, it is a validated measure for classifying people as active or insufficiently active. Amireault and Godin (2015) compared the GLTEQ results with participants' $\mathrm{VO}_{2}$ values, body fat percentages, and attendance at a fitness center, finding weak-to-medium agreement (partial $\eta^{2}=.05-.13$ ) across methods for validity. Against accelerometer data, the GLTEQ has shown medium agreement $(r=.45$; Miller, Freedson, \& Kline, 1994). Analyzing each intensity separately is uncommon, though (Homan \& Tylka, 2014).

Daily Diary. Participants completed four daily diary reports each day during the twentyone-day protocol (see Appendix E). Physical activity was one component assessed in these 
reports, requiring only a few hours of recall. The daily diary physical activity questions were based on questions from the GLTEQ, requiring participants to answer how much vigorous-, moderate-, and mild-intensity physical activity, along with sedentariness, they had completed (i.e., "Since you woke up today, how many minutes of vigorous physical activity did you engage in?", "Since the last session, how many minutes of vigorous physical activity did you engage in?). The vigorous- and moderate-intensity data was combined to create a moderate-to-vigorousintensity variable. The daily diary accounts allowed for moderate-to-vigorous-intensity, mildintensity, and sedentary intensity, frequency, and duration of physical activity to be studied. When being compared to the accelerometer data, the daily diary data was continuous. Daily diary data were dichotomized for comparisons with the GLTEQ.

\section{Power Analysis}

A power analysis was conducted using MedCalc Software (Schoonjans, 2017). The analysis incorporates information on Type I and Type II error, information for AUC, the null hypothesis value, and the ratio of sample sizes in negative and positive groups. To ensure that the intensity, duration, and frequency results are accurate, the Type I error was set at 0.05 and the Type II error was set at 0.20 . The AUC for the ROC curve analysis was set at 0.725 , indicating that we want to conclude with adequate or fair justification how the methodologies compare. The null hypothesis value was set to 0.5 , and the ratio for the sample size across negative and positive groups was set to 1 , indicating equal group sizes. With these parameters, a sample size of $\mathrm{N}=48$ is needed for sufficient power $(>.80)$ to analyze differences across the methodologies.

\section{Preliminary Analyses}

Using SPSS Version 21, univariate, bivariate, and multivariate problems were assessed. Variables that had a skew score above 3.2 underwent a square root transformation. The 
windsorizing technique was implemented to retain extreme data points (e.g., Ghosh \& Vogt, 2012). After ensuring all assumptions were met, scale reliability statistics were computed. As the study spanned 21 days, Little's Missing Completely at Random (MCAR) test was conducted to examine missingness in the daily diary data. If Little's MCAR test resulted in a non-significant statistic, and if participants had data for at least 4 of the 7 days per week, estimation maximization was conducted to impute data. All moderate-to-vigorous-intensity, mild-intensity, and sedentary behavior daily diary data were missing completely at random, so values were imputed. To keep as many cases as possible, if a participant had fewer than 4 days of data in a week, their non-imputed sum was utilized. No significant differences emerged when analyses were conducted with data for at least 3 days, so the non-imputed sums were kept.

\section{Receiver Operator Characteristic Curve Analyses}

Receiver operator characteristic (ROC) curve analyses examine the agreement between measures (Fawcett, 2005; Zou, O’Malley, \& Mauri, 2007). Thirty-four ROC curve analyses were conducted in the current study based on the availability of data for intensity, frequency, and duration of physical activity.

1. Week 2 Moderate-to-vigorous physical activity intensity [accelerometer and GLTEQ]

2. Week 3 Moderate-to-vigorous physical activity intensity [accelerometer and GLTEQ]

3. Week 2 Moderate-to-vigorous physical activity intensity [accelerometer and daily diary]

4. Week 3 Moderate-to-vigorous physical activity intensity [accelerometer and daily diary]

5. Week 2 Moderate-to-vigorous physical activity intensity [daily diary and GLTEQ]

6. Week 3 Moderate-to-vigorous physical activity intensity [daily diary and GLTEQ]

7. Week 2 Mild physical activity intensity [accelerometer and GLTEQ]

8. Week 3 Mild physical activity intensity [accelerometer and GLTEQ] 
9. Week 2 Mild physical activity intensity [accelerometer and daily diary]

10. Week 3 Mild physical activity intensity [accelerometer and daily diary]

11. Week 2 Mild physical activity intensity [daily diary and GLTEQ]

12. Week 3 Mild physical activity intensity [daily diary and GLTEQ]

13. Week 2 Sedentary intensity [accelerometer and daily diary]

14. Week 3 Sedentary intensity [accelerometer and daily diary]

15. Week 2 Moderate-to-vigorous physical activity frequency [accelerometer and daily diary]

16. Week 3 Moderate-to-vigorous physical activity frequency [accelerometer and daily diary]

17. Week 2 Mild physical activity frequency [accelerometer and daily diary]

18. Week 3 Mild physical activity frequency [accelerometer and daily diary]

19. Week 2 Sedentary physical activity frequency [accelerometer and daily diary]

20. Week 3 Sedentary physical activity frequency [accelerometer and daily diary]

21. Week 2 Moderate-to-vigorous physical activity duration [accelerometer and GLTEQ]

22. Week 3 Moderate-to-vigorous physical activity duration [accelerometer and GLTEQ]

23. Week 2 Moderate-to-vigorous physical activity duration [accelerometer and daily diary]

24. Week 3 Moderate-to-vigorous physical activity duration [accelerometer and daily diary]

25. Week 2 Moderate-to-vigorous physical activity duration [daily diary and GLTEQ]

26. Week 3 Moderate-to-vigorous physical activity duration [daily diary and GLTEQ]

27. Week 2 Mild physical activity duration [accelerometer and GLTEQ]

28. Week 3 Mild physical activity duration [accelerometer and GLTEQ]

29. Week 2 Mild physical activity duration [accelerometer and daily diary]

30. Week 3 Mild physical activity duration [accelerometer and daily diary]

31. Week 2 Mild physical activity duration [daily diary and GLTEQ] 
32. Week 3 Mild physical activity duration [daily diary and GLTEQ]

33. Week 2 Sedentary duration [accelerometer and daily diary].

34. Week 3 Sedentary duration [accelerometer and daily diary]

To conduct ROC curve analyses, specificity, or the rate at which data are inaccurate, is plotted on the $\mathrm{x}$-axis and sensitivity, or the rate at which the measure is accurate, is plotted on the y-axis (Zou et al., 2007). Examining these components allows for discrimination of each measure's data. To evaluate the agreement of measures, the area under the curve (AUC) is analyzed, which averages the accuracy of each measure (Zou et al., 2007). An AUC of 1 suggests complete agreement, 0.90 indicates excellent agreement, 0.80 marks good agreement, 0.70 indicates fair agreement, 0.60 notes poor agreement, and an AUC of 0.5 indicates chance agreement (Zou et al., 2007). An AUC below 0.5 suggests inaccuracy (Fawcett, 2005). The state variable, which was the "gold standard" variable, was always dichotomous. The test variable, which was the data being compared to the standard, was always continuous. Means and standard deviations for intensity of physical activity is listed in Table 2, those for frequency of physical activity are listed in Table 3, and descriptive statistics for duration of physical activity are provided in Table 4.

\section{Results}

\section{Research Question 1: Agreement across Subjective and Objective Measures}

Intensity. To study intensity, an activity score was generated, which has been done in past research (e.g., Ashok, Kharche, Raju, \& Godbole, 2016) for all data points. For all comparisons, the accelerometer was considered to be the "gold standard", with the daily diary viewed as more accurate than the GLTEQ. Because the GLTEQ does not ask about sedentary behavior, only the agreement between accelerometer and daily diary was analyzed for sedentary 
behavior. Mean differences using Bonferroni-adjusted pairwise comparisons and single measures intraclass correlations were conducted to further understand the agreement across measures. See Table 5 for the intraclass correlation coefficients and see Table 6 for all Intensity ROC curve statistics.

Number of minutes of moderate-to-vigorous-intensity physical activity was calculated for each week, which was then multiplied by 6 , which marks an average MET value for moderateto-vigorous-intensity physical activity (Ashok et al., 2016). This product was then multiplied by 150, mapping on to recommendations to engage in 150 minutes of moderate-to-vigorousintensity physical activity each week. Based on recommendations, 900 was the cut-off for the dichotomized variable. For the "gold standard", activity scores of 900 or above were marked as " 1 " and scores under 900 as " 0 ". Activity scores were generated rather than comparing minutes of physical activity in order to differentiate intensity and duration analyses.

For mild-intensity physical activity, the number of weekly minutes was multiplied by 2.9 , which represents the MET score of mild-intensity physical activity (Ashok et al., 2016). The activity score cut-off was 3,654, which represents 3 hours of daily mild-intensity physical activity for 7 days. Mild-intensity data that reflected an activity score of 3,654 or higher was marked with a "1" and scores below 3,654 were marked with a "0".

Lastly, for sedentary behavior, the number of weekly minutes was multiplied by 1.09 , which represents a sedentary MET score, to generate activity scores. Activity scores were generated for the accelerometer, GLTEQ, and daily diary. The GLTEQ does not provide data on sedentary behavior. For sedentary behavior, an activity score at or above 3,525.06 was scored as a "1" and any score below 3,525.06 was marked as a " 0 ". This cut-off was chosen based on Matthews and colleagues (2008) conclusion that adults spend 7.7 daily hours in a sedentary 
behavior.

Week 2 Intensity. For moderate-to-vigorous-intensity physical activity, there was poor agreement between the accelerometer and GLTEQ (AUC $=0.596,95 \%$ CI $[0.413,0.778])$. Only chance agreement was detected between the accelerometer and daily diary data $(\mathrm{AUC}=0.539$, $95 \% \mathrm{CI}[0.348,0.730])$, and the daily diary and GLTEQ data, $(\mathrm{AUC}=0.535,95 \% \mathrm{CI}[0.352$, 0.718]). Using Bonferroni-adjusted pairwise comparisons, mean differences were assessed. The comparisons did not detect any differences between the mean activity scores of the accelerometer $(M=1752.63)$, GLTEQ $(M=1106.05)$, and daily diary $(M=1707.18)$. The single measures intraclass correlations for activity scores of moderate-to-vigorous-intensity physical activity were non-significant all non-significant across the three measures.

Among mild-intensity physical activity, slightly worse than chance agreement emerged between accelerometer and GLTEQ data $(\mathrm{AUC}=0.48495 \% \mathrm{CI}[0.247,0.722])$, but a poor-tofair agreement was found between the accelerometer and daily diary data $(\mathrm{AUC}=0.671,95 \% \mathrm{CI}$ $[0.429,0.914])$. Poor agreement emerged between the dichotomized daily diary and GLTEQ data $(\mathrm{AUC}=0.624,95 \% \mathrm{CI}[0.438,0.809])$. Studying mean differences with Bonferroni-adjusted pairwise comparisons, the accelerometer mean activity score $(M=5613.56)$ was significantly higher $(p<0.001)$ than the daily diary mean activity score $(M=1757.20)$, which was significantly higher $(p<0.001)$ than the GLTEQ mean activity score $(M=477.36)$. The single measures intraclass correlations for activity scores of mild-intensity physical activity across the three measures were non-significant.

For sedentariness, only chance agreement was found between the accelerometer and daily diary data $(\mathrm{AUC}=0.509,95 \% \mathrm{CI}[0.315,0.703])$. Sedentary intensity data were only available 
from the accelerometer and daily diary accounts, so no other ROC curve analyses were conducted. The pairwise comparison with the Bonferroni adjustment detected a significant mean difference $(p<0.001)$ between the mean activity scores of the accelerometer $(M=3830.23)$ and daily diary $(M=1187.39)$. The single measures intraclass correlation for accelerometer and daily diary sedentariness was non-significant.

Week 3 Intensity. Between accelerometer and GLTEQ moderate-to-vigorous-intensity physical activity data, chance agreement emerged $(\mathrm{AUC}=0.514,95 \% \mathrm{CI}[0.335,0.693])$. There was poor agreement between the moderate-to-vigorous-intensity accelerometer and daily diary data $(\mathrm{AUC}=0.617,95 \% \mathrm{CI}[0.421,0.814])$. Comparing daily diary and GLTEQ data for this intensity, there was poor agreement $(\mathrm{AUC}=0.592,95 \% \mathrm{CI}[0.407,0.776])$. Comparing the mean activity scores of the accelerometer $(M=1611.88)$, GLTEQ $(M=1114.41)$, and daily diary $(M=$ 1980.89), the Bonferroni-adjusted pairwise comparisons did not detect any significant mean differences. Comparing the three measures, the single measures intraclass correlations for duration of moderate-to-vigorous-intensity physical activity were non-significant across the three measures.

For mild-intensity physical activity, worse than chance agreement emerged between the accelerometer and GLTEQ data $(\mathrm{AUC}=0.447,95 \% \mathrm{CI}[0.272,0.623])$, and chance-to-poor agreement was found between the accelerometer and daily diary data $(\mathrm{AUC}=0.573,95 \% \mathrm{CI}$ $[0.330,0.816])$. There was good agreement between the mild-intensity daily diary data, which was treated as the "gold standard", and the GLTEQ (AUC $=0.794,95 \%$ CI $[0.646,0.942])$. Studying the pairwise comparisons, the three means were all significantly different. The mean activity score of the accelerometer $(M=5474.02)$ was significantly different $(p<0.001)$ from the mean activity score of the daily diary $(M=1946.05)$, which was significantly different $(p<0.01)$ 
from the mean activity score of the GLTEQ $(M=578.43)$. The single measures intraclass correlations for duration of mild-intensity physical activity were all non-significant.

Similar to the findings from Week 2, there was worse than chance agreement between the sedentary accelerometer and daily diary data $(\mathrm{AUC}=0.331,95 \% \mathrm{CI}[0.136,0.526])$. No other ROC curve analyses were conducted for sedentary intensity. The Bonferroni-adjusted pairwise comparison found a significant mean difference $(p<0.001)$ between the mean activity scores of the accelerometer $(M=3778.82)$ and daily diary $(M=1454.58)$. The single measures intraclass correlation for duration of sedentariness was non-significant.

Frequency. For frequency, the number of active days within the past 7 days was studied. Number of active days was assessed for moderate-to-vigorous-intensity, mild-intensity, and sedentary behaviors. The GLTEQ does not provide frequency data, so agreement was only studied between the accelerometer and daily diary. Additionally, data on sedentary behavior is not available from the GLTEQ. All ROC curve statistics for Frequency are listed in Table 7. The Bonferroni adjustment was employed to assess pairwise comparisons, along with single measures intraclass correlations being conducted to study agreement across measures. See Table 5 for the intraclass correlation coefficients.

Accelerometer data, which were considered the "gold standard", was dichotomized, with " 1 " indicating participants engaged in physical activity for 7 days. A " 0 " indicated participants did not engage in physical activity on all 7 days. These dichotomizing rules were applied to moderate-to-vigorous and mild physical activity. The same rule was used for sedentariness, such that participants received a " 1 " if participants had engaged in sedentary behavior for all 7 days and a " 0 " if participants did not engage in sedentary behavior on all 7 days. Between the two subjective measures, the daily diary was treated as the "gold standard". 
Week 2 Frequency. Analyzing the moderate-to-vigorous physical activity frequency for Week 2, there was worse than chance agreement between the accelerometer and daily diary data $(\mathrm{AUC}=0.480,95 \% \mathrm{CI}[0.238,0.721])$. This is the only ROC curve generated for frequency. Pairwise comparisons were analyzed for mean differences. Using the Bonferroni adjustment, pairwise comparison results indicated there was a significant difference $(p<0.05)$ between the accelerometer $(M=4.76)$ and daily diary $(M=3.36)$ means. The single measures intraclass correlation for the two frequency measures of moderate-to-vigorous-intensity physical activity was non-significant.

For frequency of mild-intensity physical activity, there was good agreement between accelerometer and daily diary data $(\mathrm{AUC}=0.836,95 \% \mathrm{CI}[0.688,0.983])$. This is the only ROC curve generated for frequency. The Bonferroni-adjusted pairwise comparison detected a significant difference $(p<0.001)$ between the accelerometer $(M=6.62)$ and daily diary $(M=$ 4.38) means. The mild-intensity physical activity single measures intraclass correlation was nonsignificant.

The agreement for sedentary frequency data between the accelerometer and daily diary was poor-to-fair $(\mathrm{AUC}=0.686,95 \% \mathrm{CI}[0.389,0.984])$. This is the only ROC curve generated for frequency. The pairwise comparison found a significant difference $(p<0.001)$ between the accelerometer $(M=6.62)$ and the daily diary $(M=3.36)$ means using the Bonferroni adjustment. The single measures intraclass correlation for sedentary frequency was non-significant.

Week 3 Frequency. For moderate-to-vigorous-intensity physical activity frequency, poor agreement emerged between the accelerometer and daily diary data $(\mathrm{AUC}=0.603,95 \% \mathrm{CI}$ $[0.322,0.885])$. This is the only ROC curve generated for frequency. The Bonferroni-adjusted pairwise comparison did detect a significant mean difference $(p<0.01)$ between the 
accelerometer $(M=4.66)$ and the daily diary $(M=3.29)$ means. The single measures intraclass correlation for moderate-to-vigorous-intensity physical activity frequency was non-significant.

The ROC curve analysis for mild-intensity physical activity frequency suggested chance agreement between the accelerometer and daily diary data $(\mathrm{AUC}=0.546,95 \% \mathrm{CI}[0.339$, 0.753]). This is the only ROC curve generated for frequency. Using the Bonferroni adjustment, the pairwise comparison concluded a significant mean difference existed $(p<0.001)$ between the accelerometer $(M=6.47)$ and the daily diary $(M=4.58)$ means. For frequency of mild-intensity physical activity, the single measures intraclass correlation was non-significant.

Comparing sedentary frequency accelerometer and daily diary data, only chance-to-poor agreement emerged $(\mathrm{AUC}=0.583,95 \% \mathrm{CI}[0.333,0.833])$. This is the only ROC curve generated for frequency. Analyzing the pairwise comparison, a significant mean difference emerged $(p<0.001)$ between the accelerometer $(M=6.46)$ and daily diary $(M=4.43)$ means. The sedentary frequency single measures intraclass correlation was non-significant.

Duration. To study duration of physical activity, the number of weekly minutes spent engaging in moderate-to-vigorous-intensity physical activity, mild-intensity physical activity, and sedentary behavior was assessed. Duration data were available from the accelerometer, GLTEQ, and daily diary. All duration ROC Curve statistics are contained in Table 8 . The Bonferroni-adjusted pairwise comparisons and single measures intraclass correlations were additionally analyzed to study agreement across measures. See Table 5 for the intraclass correlation coefficients.

Accelerometer data were dichotomized, with " 1 " indicating participants fulfilled the recommended amount of moderate-to-vigorous or mild physical activity and a " 0 " indicating participants engaged in less than the recommended amount of physical activity. For moderate-to- 
vigorous physical activity, participants needed to engage in 150 minutes of weekly physical activity. For mild-intensity physical activity, participants were required to have completed 1,260 minutes of weekly mild physical activity to earn a "1". Lastly, when studying sedentariness, a " 1 " indicated participants engaged in at least 3,234 minutes of sedentary behavior and a " 0 " indicated participants had a total sedentary time less than 3,234 minutes. When comparing the two subjective measures, the daily diary was considered to be the "gold standard".

Week 2 Duration. The agreement for duration of moderate-to-vigorous-intensity physical activity was poor between the accelerometer and daily diary $(\mathrm{AUC}=0.598,95 \% \mathrm{CI}[0.418$, 0.779]). There was chance agreement between the duration of moderate-to-vigorous-intensity physical activity accelerometer and daily diary data $(\mathrm{AUC}=0.539,95 \% \mathrm{CI}[0.348,0.730])$, and between the subjective measures $(\mathrm{AUC}=0.537,95 \% \mathrm{CI}[0.356,0.719])$. Assessing the accelerometer $(M=292.11)$, GLTEQ $(M=184.34)$, and daily diary $(M=284.53)$ means with pairwise comparisons, the Bonferroni-adjusted results showed no significant differences between the three measures' means. The single measures intraclass correlations for moderate-to-vigorousintensity physical activity across the three measures were non-significant.

For duration of mild-intensity physical activity, the agreement between the accelerometer and GLTEQ was slightly worse than chance $(\mathrm{AUC}=0.484,95 \% \mathrm{CI}[0.247,0.722])$. Poor-to-fair agreement resulted between the duration of mild-intensity accelerometer and daily diary data $(\mathrm{AUC}=0.671,95 \% \mathrm{CI}[0.429,0.914]$, and poor agreement emerged between the daily diary and GLTEQ data $(\mathrm{AUC}=0.624,95 \%$ CI $[0.438,0.809])$. Studying the measures' means with pairwise comparisons, the Bonferroni method deduced that each measure was significantly different from the other $(p<0.001)$, with the accelerometer mean $(M=1935.71)$ significantly higher than the daily diary mean $(M=605.93)$, which scored significantly higher $(p<0.001)$ 
than the GLTEQ mean $(M=164.61)$. The single measures intraclass correlations for mildintensity physical activity across the three measures were all non-significant.

The agreement between the accelerometer and daily diary sedentary data was chance $(\mathrm{AUC}=0.537,95 \% \mathrm{CI}[0.340,0.734])$. Sedentary activity data were only collected from the accelerometer and daily diary, so no other ROC curves were conducted. The Bonferroni adjustment that was used to compare means found a significant difference $(p<0.001)$ between the accelerometer $(M=3513.98)$ and daily diary $(M=1278.36)$ means. The single measures intraclass correlation for accelerometer and daily diary sedentariness was non-significant.

Week 3 Duration. A chance relationship emerged between the duration of moderate-tovigorous-intensity physical activity accelerometer and GLTEQ data (AUC $=0.514,95 \% \mathrm{CI}$ $[0.335,0.693])$. The relationship between the accelerometer and daily diary moderate-tovigorous-intensity physical activity data represented poor agreement $(\mathrm{AUC}=0.617,95 \% \mathrm{CI}$ $[0.421,0.814])$. Poor agreement was also established between the two subjective measures for duration of moderate-to-vigorous-intensity physical activity $(\mathrm{AUC}=0.592,95 \% \mathrm{CI}[0.407$, 0.776]). Assessing the pairwise comparisons, the Bonferroni adjustment concluded no significant differences existed between the accelerometer $(M=268.65)$, GLTEQ $(M=185.74)$, and daily diary $(M=330.15)$ means. The single measures intraclass correlations for duration of moderateto-vigorous-intensity physical activity were non-significant across the three measures.

The agreement between duration of mild-intensity physical activity accelerometer and GLTEQ data is slightly worse than chance $(\mathrm{AUC}=0.447,95 \% \mathrm{CI}[0.272,0.623])$, and the agreement between the accelerometer and daily diary data was chance-to-poor (AUC $=0.573$, $95 \%$ CI $[0.330,0.816])$. With the daily diary data dichotomized to be the "gold standard" for mild-intensity physical activity, the agreement between the two subjective measures was good 
$(\mathrm{AUC}=0.794,95 \% \mathrm{CI}[0.646,0.942])$. The Bonferroni adjustment was employed to study the pairwise comparisons, with the accelerometer $(M=1887.60)$ mean significantly higher $(p<$ $0.001)$ than the daily diary $(M=670.05)$ mean, which was significantly higher $(p<0.01)$ than the GLTEQ $(M=199.46)$. For mild-intensity physical activity, the single measures intraclass correlations were non-significant.

For sedentary behavior, the agreement between the accelerometer and daily diary data at Week 3 was very low, representing worse than chance agreement $(\mathrm{AUC}=0.363,95 \% \mathrm{CI}[0.164$, 0.562]). Sedentary activity duration was only available for the accelerometer and daily diary, so no other ROC curve analyses were conducted. The Bonferroni-adjusted pairwise comparisons concluded a significant difference existed $(p<0.001)$ between the accelerometer $(M=3412.84)$ and daily diary $(M=1272.64)$ means. The single measures intraclass correlation for sedentary behavior, which accounted for accelerometer and daily diary data, was non-significant.

\section{Research Question 2: Age Differences}

To assess age-based group differences, data from 21 younger adults aged 18-39 years (Group 1) and 35 middle-aged and older adults aged 40-72 years (Group 2) was studied using the statistical and graphics software NCSS 12 (Hintze, 1988) was utilized. This software has been used in other publications (e.g., Spindler et al., 2015; Vavra, Saadeh, Rosen, Uptigrove, \& Srkalovic, 2013). All thirty-four of the ROC curve analyses were conducted again in NCSS 12, with the added capability of comparing groups. The AUC scores were compared based on zscores. In general, both age groups reported in such a manner that poor-to-fair agreement emerged. Significant differences are discussed below. See Table 9 for all AUC statistics for the age-based ROC curve analyses.

Intensity. Week 3 sedentary activity scores were worse than chance for both younger 
adults $(\mathrm{AUC}=0.375,95 \% \mathrm{CI}[-0.178,0.748])$ and middle-aged and older adults $(\mathrm{AUC}=0.289$, 95\% CI $[0.032,0.510])$. These AUC scores note the significant inaccuracy among all adults for this intensity.

Frequency. When assessing frequency of mild-intensity physical activity between the accelerometer and daily diary at Week 2, middle-aged and older adults showed good agreement in their reports $(\mathrm{AUC}=0.818,95 \% \mathrm{CI}[0.515,0.940])$, but younger adults' reports were more inaccurate than chance $(\mathrm{AUC}=0.467,95 \% \mathrm{CI}[0.170,0.686])$, which resulted in a significant AUC difference $\left(\mathrm{AUC}_{\text {difference }}=0.352, \mathrm{z}\right.$-score $\left.=2.123, p<.05\right)$. Significant inaccuracy was also demonstrated for frequency of Week 3 moderate-to-vigorous-intensity for younger adults (AUC $=0.000)$ and middle-aged and older adults $(\mathrm{AUC}=0.292,95 \% \mathrm{CI}[-0.128,0.618])$. The agreement, thus, was below chance for all adults.

Duration. All adults responded with inaccuracy for duration of Week 3 sedentariness. Younger adults $(\mathrm{AUC}=0.400,95 \% \mathrm{CI}[-0.126,0.750])$ and middle-aged and older adults (AUC $=0.360,95 \%$ CI $[0.085,0.584])$ both had scores below chance.

\section{Research Question 3: Sex Differences}

NCSS 12 (Hintze, 1988), the same statistical and graphics software utilized to assess age differences, was used to study sex differences. Thirty-four additional ROC curve analyses were conducted with sex included as the grouping variable. As stated in the Methods section, the majority of the sample was female, with 43 female (Group 1) and 13 male (Group 2) participants. Generally, males and females had similar agreement in their intensity, frequency, and duration of physical activity. Significant sex differences are discussed below. See Table 10 for all AUC statistics for the sex-based ROC curve analyses.

Intensity. For intensity of sedentariness at Week 2, females showed worse than chance 
agreement $(\mathrm{AUC}=0.439,95 \% \mathrm{CI}[0.197,0.630])$ and males demonstrated excellent agreement $(\mathrm{AUC}=0.889,95 \% \mathrm{CI}[0.171,0.990])$, resulting in a significant $\mathrm{AUC}$ difference $\left(\mathrm{AUC}_{\text {difference }}=\right.$ $0.450, \mathrm{z}$-score $=2.592, p<.01)$. Additionally, there was a significant difference between the accelerometer and GLTEQ in Week 2 mild-intensity activity scores between females (AUC = $0.550,95 \% \mathrm{CI}[0.283,0.738])$ and males $(\mathrm{AUC}=0.056,95 \% \mathrm{CI}[-0.054,0.163])$, as detected from AUC scores $\left(\mathrm{AUC}_{\text {difference }}=0.494\right.$, z-score $\left.=3.83, p<.001\right)$. At Week 3, in comparison to accelerometer data, males were completely inaccurate at noting their mild-intensity activity on the GLTEQ $(\mathrm{AUC}=0.000)$ and on daily diary accounts $(\mathrm{AUC}=0.000)$. This complete inaccuracy also occurred in male accounts of their Week 3 sedentariness $(A U C=0.000)$. At Week 3 sedentariness, females also experienced inaccuracy in their reports (AUC $=0.374,95 \%$ CI $[0.130,0.575])$.

Frequency. At Week 2, females showed chance agreement for frequency of moderate-tovigorous-intensity physical activity $(\mathrm{AUC}=0.534,95 \% \mathrm{CI}[0.280,0.718])$, but males' reports demonstrated high inaccuracy $(\mathrm{AUC}=0.056,95 \% \mathrm{CI}[-0.054,0.163])$, which resulted in a significant group difference statistic $\left(\mathrm{AUC}_{\text {difference }}=0.478, \mathrm{z}\right.$-score $\left.=3.82, p<.01\right)$. Frequency of sedentary behavior at Week 2 for males $(\mathrm{N}=11)$ resulted in inaccuracy $(\mathrm{AUC}=0.389,95 \% \mathrm{CI}$ $[0.090,0.624])$, but the frequency of sedentary behavior at Week 3 for males $(\mathrm{N}=7)$ showed absolute inaccuracy $(\mathrm{AUC}=0.000)$. Males also showed complete inaccuracy in reporting their frequency of Week 3 mild-intensity physical activity $(\mathrm{AUC}=0.000)$. Females also experienced inaccurate-to-chance agreement $(\mathrm{AUC}=0.352-0.526)$ for frequency of Week 3 moderate-tovigorous-intensity, mild-intensity, and sedentariness.

Duration. A significant sex difference emerged for Week 2 intensity of mild-intensity physical activity between the accelerometer and GLTEQ. Females demonstrated chance 
agreement $(\mathrm{AUC}=0.550,95 \% \mathrm{CI}[0.283,0.738])$; whereas males showed high inaccuracy (AUC $=0.056,95 \%$ CI $[-0.054,0.163])$. Furthermore, although males answered with perfect agreement for duration of Week 2 sedentariness $(\mathrm{AUC}=1.000)$, males answered with complete inaccuracy at Week 3 sedentariness $(\mathrm{AUC}=0.000)$. Females also answered with inaccuracy for Week 3 sedentariness $(\mathrm{AUC}=0.402,95 \% \mathrm{CI}[0.156,0.601])$. Both males' $(\mathrm{AUC}=0.400,95 \% \mathrm{CI}[-$ $0.147,0.760])$ and females' $(\mathrm{AUC}=0.452,95 \% \mathrm{CI}[0.152,0.676])$ scores were below chance for duration of Week 3 moderate-to-vigorous-intensity physical activity between the accelerometer and daily diary.

\section{Research Question 4: Motivation Differences}

Thirty-four additional ROC curve analyses were conducted with motivation included as the grouping variable. Participants scoring below the mean $(M=51.04)$ for motivation were considered having low-to-moderate motivation (scored as a "1") and those with scores above the mean were classified as having moderate-to-high motivation (scored as a "2"). Scores were generally similar across differently-motivated participants. Significant differences are discussed below. See Table 11 for all AUC statistics for the motivation-based ROC curve analyses.

Intensity. Week 2 accelerometer and daily diary mild-intensity activity scores among participants with low-to-moderate intrinsic motivation showed good agreement $(\mathrm{AUC}=0.808$, 95\% CI $[0.330,0.956])$, but participants with moderate-to-high intrinsic motivation were entirely incorrect $(\mathrm{AUC}=0.000)$. Week 2 accelerometer and GLTEQ data for mild-intensity physical activity was worse than chance for both the low-to-moderate intrinsic motivation group (AUC = $0.463,95 \% \mathrm{CI}[0.128,0.703)$ and the moderate-to-high intrinsic motivation group (AUC $=$ $0.375,95 \%$ CI $[0.195,0.531])$. At Week 3 , intensity of sedentary behaviors for participants with low-to-moderate intrinsic motivation $(\mathrm{AUC}=0.148,95 \% \mathrm{CI}[-0.056,0.341])$ and participants 
with moderate-to-high intrinsic motivation $(\mathrm{AUC}=0.527,95 \% \mathrm{CI}[0.148,0.771])$ resulted in a significant group difference $\left(\mathrm{AUC}_{\text {difference }}=0.379, \mathrm{z}\right.$-score $\left.=1.985, p<.05\right)$. No other group differences for intensity emerged.

Frequency. At Week 2, both groups with low-to-moderate intrinsic motivation (AUC = $0.447,95 \% \mathrm{CI}[0.150,0.669])$ and moderate-to-high intrinsic motivation $(\mathrm{AUC}=0.352,95 \% \mathrm{CI}$ $[-0.072,0.667])$ showed inaccuracy. This inaccuracy was also demonstrated for Week 2 sedentariness among those with low-to-moderate intrinsic motivation (AUC $=0.472,95 \%$ CI [$0.441,0.905])$ and moderate-to-high intrinsic motivation $(\mathrm{AUC}=0.347,95 \% \mathrm{CI}[0.015,0.610])$. The only significant group difference emerged for Week 3 sedentariness. Participants with moderate-to-high intrinsic motivation answered with poor agreement $(\mathrm{AUC}=0.639,95 \% \mathrm{CI}$ $[0.272,0.844])$, but those with low-to-moderate intrinsic motivation answered the daily diary accounts with high inaccuracy $(\mathrm{AUC}=0.250,95 \% \mathrm{CI}[0.038,0.441])$.

Duration. Between accelerometer and daily diary data for Week 2 duration of mildintensity physical activity, although participants with low-to-moderate intrinsic motivation established good agreement in their accounts $(\mathrm{AUC}=0.808,95 \% \mathrm{CI}[0.330,0.956])$, participants with moderate-to-high intrinsic motivation were completely inaccurate $(\mathrm{AUC}=0.000)$. Inaccuracy was demonstrated at Week 2 mild-intensity physical activity between the accelerometer and GLTEQ for participants with low-to-moderate intrinsic motivation (AUC = $0.463,95 \% \mathrm{CI}[0.1280 .703])$ and moderate-to-high intrinsic motivation (AUC $=0.375,95 \% \mathrm{CI}$ $[0.195,0.531])$. No other group differences emerged for duration of physical activity as a result of intrinsic motivation.

\section{Discussion}

Physical activity is a positive lifestyle behavior linked to many physical and 
psychological health benefits (Brunes et al., 2013; Davies et al., 2012; Schoenborn et al., 2013; WHO, 2019). Although physical activity is important across the life span, research indicates that the majority of U.S. adults are not meeting the physical activity recommendations regarding intensity, duration, and frequency (Schoenborn et al., 2013; WHO, 2019). Not only are adults not engaging in the proper intensity, duration, and frequency of physical activity, but the current physical activity literature contains gaps. For example, often only "physical activity" or "physical activity level" is discussed in the literature without proper specification for which component (e.g., intensity, frequency, duration) is studied. Additionally, although some studies have assessed associations across measures, only select analyses have been employed. Considering the current state of the literature, the purpose of the current study was twofold: to significantly add to the current literature by focusing on intensity, frequency, and duration of physical activity and to extend the current literature by utilizing ROC curve analyses to assess agreement across subjective and objective measures of the three physical activity components.

The current study used data from two separate weeks to assess the agreement between the accelerometer, GLTEQ, and daily diary data for the three physical activity components (intensity, frequency, and duration). Considering intensity, frequency, and duration of physical activity, it was hypothesized that both the GLTEQ and daily diary would over-report the three components of physical activity in comparison to the accelerometer. However, it was hypothesized that the daily diary, and not the GLTEQ, would exhibit the strongest agreement to the accelerometer data. Comparing the two subjective measures, it was hypothesized that the GLTEQ and daily diary would strongly agree. Additionally, it was projected that only group differences for intrinsic motivation would emerge. See Appendix F for all hypotheses and results. 


\section{Intensity of Physical Activity}

Although the current validation literature provides insight on most physical activity intensities, a large gap exists for moderate-to-vigorous-intensity physical activity. Moderate-tovigorous-intensity physical activity is especially important to track due to the increase in benefits from the high intensity (Powell, Paluch, \& Blair, 2011; Wen et al., 2011). Specific to moderateto-vigorous-intensity physical activity, the current study found only chance or poor agreement between the various measures at both weeks. Looking at the activity score means, participants frequently under-reported their moderate-to-vigorous-intensity physical activity, although daily diary accounts of moderate-to-vigorous physical activity at Week 3 were over-estimated. Although the subjective measures did not strongly agree with the objective data, mean differences were not statistically significant. Thus, adults experience some insufficiency in accurately reporting their higher-intensity physical activity. This highlights the importance of teaching participants about moderate-to-vigorous-intensity activities, as well the need for researchers to consider other methods of tracking physical activity.

Slightly better agreement existed between some measures of mild-intensity physical activity. The agreement between the accelerometer and daily diary at Week 2 was fair and the agreement between the daily diary and GLTEQ was good at Week 3. These higher rates of agreement suggest two important points. First, potentially due to the frequent sampling of physical activity (Nehrkorn et al., 2018), daily diary accounts show stronger agreement than other subjective measures to the objective data. Second, stronger agreement emerged between the subjective measures potentially because they were both based on personal accounts. These results support the current hypotheses but contradict the findings from the current literature (e.g., Sabia et al., 2014; Vandelanotte et al., 2005). However, due to the inconsistencies across Weeks 
2 and 3 for mild-intensity physical activity, possibly a better explanation is that daily diary accounts have the potential to better reflect objective data, but it is not guaranteed. If daily diary responses are delayed or late, for example, participants may experience more difficulty in recalling the intensity of their physical activity (Hallal et al., 2012; Sirard et al., 2013). Between the accelerometer and GLTEQ, mild-intensity physical activity AUC statistics were both slightly below the cut-off for chance agreement. These low scores for the GLTEQ-accelerometer analyses support the current study's hypothesis that the weakest agreement would exist between these two measures. It is important to note that all of the pairwise comparisons ended in significant differences across the means, with participants consistently under-reporting their mild-intensity physical activity. The ROC curve analyses assess each individual data point across the two measures and are not based on the measure's mean. Nonetheless, the ROC curve analyses' results are encouraging, noting the quality of subjective accounts of mild-intensity physical activity between the daily diary-accelerometer and daily diary-GLTEQ analyses.

The results for sedentary behavior, however, noted a complete lack of agreement between the accelerometer and daily diary. With the accelerometer and daily diary being the only two measures to study sedentariness, only two ROC curve analyses were conducted. Across the two analyses, chance agreement emerged at Week 2 and the agreement at Week 3 was worse than chance. In comparison to the accelerometer data, participants consistently underestimated their sedentariness in their daily diary accounts. Potentially participants were not critically considering their sedentariness each day, but just providing an estimate of the duration they spent in this intensity. Additionally, the social desirability bias may have affected participants' answers. With the study focusing on increasing physical activity, participants possibly wanted to hide some of their sedentariness from researchers. Regardless, the low AUC scores and significant 
underestimation suggests adults may have the most difficult time labeling the sedentary-intensity activities. In the current literature, one study does note a weak association across measures (Kozey-Keadle et al., 2010), but few other studies have assessed sedentariness. A stronger emphasis on sedentariness is needed, educating people on the various activities that constitute sedentariness. Better measures are also needed to assess sedentariness. Adults may be engaging in such a large amount of sedentary behaviors that using a traditional questionnaire or daily diary measure becomes inadequate.

Although low levels of agreement existed across the various analyses, the agreement between the accelerometer and GLTEQ was generally lower than the agreement found for the accelerometer-daily diary and daily diary-GLTEQ analyses. Thus, even waiting one week to recall intensity may be too difficult for participants to accurately report their activity. The fairly consistent poor and low agreement for each intensity suggests people have difficulty noting the intensity of their physical activity. The weak agreement occurred due to the fairly consistent under-reporting of activities, which does not support the current study's hypothesis. There may be serious implications of under- or over-reporting physical activity for some people. For example, if one living with cardiovascular disease under-reports their physical activity, they could be putting themselves at risk of overexertion, resulting in deleterious health outcomes. Future research, education programs, and public service opportunities should focus on intensityspecific material, educating people how to accurately label intensity. Furthermore, potentially one way to increase agreement would be to teach participants strategies to increase awareness and mindfulness. Perhaps participants' sedentary reports and accelerometer-GLTEQ mildintensity reports lacked agreement due to the lack of focus on these behaviors. Measures and educational materials should describe intensity-based activities while also educating participants 
about the variability in intensity.

\section{Frequency of Physical Activity}

Although intensity-specific findings represented many chance findings, agreement was moderately strong for frequency of mild-intensity physical activity and sedentariness at Week 2 . However, only the accelerometer and daily diary measures questioned participants about their sedentary behaviors, so results focus on the agreement between these two measures. Thus, a smaller number of analyses were conducted to study frequency of physical activity. However, many studies do not investigate frequency of physical activity (e.g., Taylor-Piliae et al., 2010), so the current study's comparisons between the accelerometer and daily diary provide important information and implications for future studies.

At Week 2, agreement was good for frequency of mild-intensity activity, fair for frequency of sedentariness, and chance for moderate-to-vigorous-intensity physical activity. At Week 3, however, agreement was consistently chance or poor. Notably, in comparison to the accelerometer means, frequency accounts of all intensities were underestimated in the daily diary accounts, which refutes the current study's hypothesis. Although participants were highly accurate at labeling their mild-intensity physical activity and moderately accurate at noting their sedentariness at Week 2, agreement significantly dropped at Week 3 except for moderate-tovigorous-intensity activity. Potentially participants were not as concerned with their accuracy knowing that the study was ending at the third week. To account for the improvement in reporting frequency of moderate-to-vigorous-intensity physical activity, participants may have experienced more difficulty in understanding the activities that constituted this high intensity. These inconsistencies across the two weeks are important to note because although some frequency statistics note high agreement, participants still made a considerable number of 
mistakes.

Future studies should continue to assess frequency for two reasons: it relates to important physical activity guidelines and it reinforces the importance of assessing physical activity frequency as a separate component. Although frequency of physical activity is discussed in the current guidelines (e.g., Schoenborn et al., 2013), it may not appear to be as important as intensity and duration due to the main recommendations focusing on minutes and intensity (e.g., 150 minutes of moderate-intensity or 75 minutes of vigorous-intensity physical activity). Although frequency results displayed some of the strongest agreement, improvement is still needed in all three intensities, but especially in the subjective accounts of frequency of moderateto-vigorous-intensity physical activity.

\section{Duration of Physical Activity}

Much of the current literature focuses on duration of physical activity (e.g., Dyrstad et al., 2014; Hagströmer et al., 2010), assessing how much physical activity is related to various outcomes (Haskell et al., 2007; Chodzko-Zajko et al., 2009). Although much of the literature has established at least weak agreement between measures, there are still inconsistencies for duration of physical activity, with some studies finding non-significant results. This non-significance predominantly occurred when participants were asked to label their activity while in different contexts, such as completing housework or walking (Hagströmer et al., 2010; Sabia et al., 2014). One benefit of the GLTEQ is that participants were provided with examples of vigorous-, moderate-, and mild-intensity activities (Godin \& Shephard, 1997), possibly allowing participants to be more conscious when engaging in such activities or assisting participants in the recall of their past week's physical activities. Within the current study, some consistent results emerged for duration of physical activity across the intensities. 
For moderate-to-vigorous physical activity, the two weeks' results showed chance-topoor agreement across the methods. The current literature, however, notes non-significant-tomedium agreement for duration of moderate-to-vigorous-intensity physical activity (Hagströmer et al., 2010; van Holle et al., 2015). Although the literature's inconsistent results stemmed from the IPAQ, a two-week recall questionnaire (Hagströmer et al., 2006), the current study's GLTEQ, a one-week recall questionnaire, and daily diary options were still marked by chance or poor agreement. Thus, adults consistently had problems in accurately reporting the amount of time they engaged in this high-intensity activity. Based on the current literature, perhaps adults find it easier to note when they are engaging in vigorous-intensity activity but become more inaccurate with the moderate-intensity activities (e.g., Hagströmer et al., 2006; Herrmann et al., 2012). Except for the Week 3 daily diary mean that over-estimated duration, participants consistently under-reported the duration of moderate-to-vigorous-intensity physical activity. The mean differences, however, were not significant. The poor agreement established by the ROC curve analyses is an important area for future intervention work to assess.

Although many inconsistencies were present in the current literature for a higher intensity, the duration of mild-intensity physical activity literature consistently notes a weak level of agreement at best (Hekler et al., 2012; Sabia et al., 2014). Results from the current study noted a bit wider agreement range. The agreement between the GLTEQ and the accelerometer was consistently worse than chance, but the other comparisons resulted in slightly stronger agreement. At Week 2, the agreement between the accelerometer and daily diary accounts, as well as the agreement between the daily diary and GLTEQ, was acceptable. Furthermore, the agreement between the daily diary and GLTEQ at Week 3 was moderately strong. These findings support my hypotheses that the strongest agreement would emerge between the 
subjective measures and between the daily diary and accelerometer. It is important to note that the GLTEQ and daily diary accounts significantly under-reported minutes of mild-intensity physical activity in comparison to the accelerometer data. Between the two subjective measures, the means from the daily diary were closer to the accelerometer data, with the largest amount of under-reporting for duration of mild-intensity physical activity occurring in GLTEQ reports.

Under-reporting also occurred in participants' daily diary accounts of their sedentariness. Although one past study concluded participants over-reported the duration of sedentariness (Hagströmer et al., 2010), multiple other studies also found that duration of sedentary behaviors was under-reported (Dyrstad et al., 2014; Kozey-Keadle et al., 2010). These consistent reports of under-reporting highlight the need to better educate adults on what sedentary behaviors may include and how to keep a more accurate log of these behaviors. Furthermore, similar to the chance results found for activity scores of sedentary behaviors, chance agreement and inaccuracy emerged for the duration of sedentary behaviors. As was previously discussed in the Discussion section, participants may be affected by the social desirability bias, leading them to under-report their sedentary time for the sake of social approval. Also, agreement may have dropped below chance due to the upcoming end of the study. Participants may have felt less connected to the study and providing accurate reports with study almost to a close. However, the Week 2 chance agreement still notes the frequent errors participants committed in reporting their sedentary behaviors. Future studies should largely focus on these behaviors, educating people on sedentariness and the negative health outcomes associated with such a lifestyle.

\section{Group Differences in Reporting Intensity, Frequency, and Duration}

Age. Physical activity and age trends have been established consistently in the current literature, suggesting that older adults engage in less physical activity (Schoenborn et al., 2013). 
Reporting behaviors based on age, however, have not been studied adequately in the current literature. The current study significantly addressed this gap, concluding that, in general, younger, middle-aged, and older adults track report their intensity, frequency, and duration of physical activity in a similar way. There was only one case when the AUC scores were significantly different between the two age groups. For Week 2 frequency of mild-intensity physical activity, middle-aged and older adults' reports strongly agreed with the accelerometer data, but younger adults' accounts established inaccurate agreement. All adults' sedentary activity scores (e.g., intensity) and duration accounts at Week 3 were generally inaccurate based on the extremely low AUC scores. This further supports the claim that all adults have difficulty reporting their sedentary behaviors. Also, as the inaccurate accounts were present at Week 3, participants potentially were less committed to accurately reporting their sedentary behaviors and duration with the study ending that week. Mild-intensity activity scores (e.g., intensity) and duration data from the accelerometer-GLTEQ analyses at Week 2 also noted inaccuracy for all adults. These findings may support the hypothesis that low agreement would exist between the accelerometer and the GLTEQ. The fact, however, that age trends were largely not found supports the current study's hypothesis and can be used to encourage higher physical activity engagement among aging adults. To note, an AUC score of 0 was present in one instance. Although complete inaccuracy could explain this score, the low sample size may also explain the score. Future work with larger samples should further explore reporting behaviors across younger, middle-aged, and older adults.

Sex. To note, the current study's sample included few males. At best, thirteen males provided data, but some data was missing, which affected the number of males included in select analyses. This limitation should be considered as the resulting sex-based differences are 
discussed below.

Various sex differences for intensity, frequency, and duration of physical activity emerged from the current study. For Week 2 sedentary intensity data, male daily diary reports of their sedentary behavior resulted in strong agreement with the accelerometer, but females reported in an inaccurate way, with an AUC score worse than chance. The sedentary activity scores at Week 3, however, noted males reported their sedentariness with complete inaccuracy, with females also demonstrating inaccuracy in their activity scores. For mild-intensity physical activity at Week 2, the agreement of female GLTEQ scores was chance in comparison to the accelerometer; whereas the agreement between males' scores was largely inaccurate. This inaccuracy in male accounts of mild-intensity physical activity was also demonstrated for Week 3 accelerometer-GLTEQ and accelerometer-daily diary activity scores. This group difference could be highlighting the inaccuracy of the subjective accounts. Based on means, the GLTEQ and daily diary both under-estimated duration of mild-intensity physical activity. For frequency of physical activity, males' sedentariness was inaccurate at both weeks, with female daily diary data charting only chance agreement with the accelerometer. Again, participants, may experience difficulty when tracking sedentariness or participants potentially felt less invested by Week 3 to accurately report their sedentariness due to the impending end of the study. Again, multiple AUC scores of 0 emerged. With the very small number of males, complete inaccuracy could explain this AUC score. Samples in future work should focus on maintaining a large number of males and females.

Looking at the means, males under-reported their frequency of sedentary behaviors at Week 3. However, examining the accelerometer data for males at both weeks, the number of days having engaged in sedentariness was 7 days without an extended range. Females, however, 
had a standard range from 0-7 days. Thus, males needed to indicate that they were active on all 7 days for agreement to be present. This finding in the accelerometer data raises the question of whether the accelerometer should be considered a "gold standard". It is a possibility that the accelerometer data was incorrect for some of the males, masking the true range for male sedentary frequency. With the small male sample size, having even a few errors could affect results. As such, future studies should focus on including a better distribution across sexes, as well as carefully choosing equipment for studies.

Motivation. Inconsistent results emerged for motivation, with participants with low-tomoderate intrinsic motivation occasionally experiencing stronger agreement over participants with moderate-to-high intrinsic motivation. Comparing mild-intensity activity scores between the accelerometer and daily diary at Week 2, those with low-to-moderate intrinsic motivation established good agreement, but participants with moderate-to-high intrinsic motivation were completely inaccurate in labeling their mild-intensity data. Perhaps participants with moderateto-high intrinsic motivation were more focused on enjoying their mild-intensity physical activity and not sufficiently considering the intensity of the activity. Participants' activity scores for sedentary intensity at Week 3 were also significantly different. For this group difference, participants with moderate-to-high intrinsic motivation showed chance agreement and participants with low-to-moderate intrinsic motivation were largely inaccurate in labeling their sedentary intensity. So, across all participants at Week 3, labeling sedentary intensity behaviors was problematic, but especially so for those with lower intrinsic motivation. For frequency of sedentariness at Week 3, there was a significant difference. Participants with moderate-to-high intrinsic motivation experienced poor agreement, but those with low-to-moderate intrinsic motivation showed inaccuracy. Across the other findings from the current study, it appears as 
though participants experienced problems reporting their sedentary behaviors across the physical activity components, with some inconsistency for mild-intensity physical activity, as well. Much of the literature focuses on vigorous-intensity and moderate-intensity physical activity (e.g., WHO, 2017b), so there is potentially more uncertainty regarding mild-intensity and sedentary behaviors.

Comparing Week 2 intensity and duration of mild-intensity physical activity data between the accelerometer and daily diary, participants with low-to-moderate intrinsic motivation showed good agreement in their reports, but those with moderate-to-high intrinsic motivation were completely inaccurate in their daily diary accounts. Analyzing the daily diary and accelerometer means, all participants under-reported their duration of mild-intensity physical activity and their activity scores, but participants with moderate-to-high intrinsic motivation under-reported their minutes of mild-intensity activity more than those with low-to-moderate intrinsic motivation. However, considering 17 participants were in the low-to-moderate intrinsic motivation group and 21 participants were part of the moderate-to-high intrinsic motivation group, the fact that all 21 participants inaccurately labeled the duration of mild-intensity physical activity is significant. This finding may support the idea that participants have a difficult time reporting mild-intensity physical activity. Again, potentially those with a higher intrinsic motivation were so focused on enjoying the physical activity that their tracking was negatively affected. As was discussed in the Age and Sex sections, the complete inaccuracy among groups may be a factor related to the small sample size. Thus, larger sample sizes are needed to further explore this work. Furthermore, as was also discussed earlier in the Discussion section, the accelerometer may potentially be providing inaccurate results for at least some people. These are some possible explanations for the striking group difference. 
To additionally explain these motivation-based outcomes, it is important to note that all participants did feel some level of intrinsic motivation. So, the participants with low-to-moderate intrinsic motivation were not absent of motivation. Furthermore, motivation was assessed at Week 1, so participants' motivators may have changed over the next two weeks. Furthermore, potentially some participants were motivated by other reasons, which were not assessed in the current study. Intrinsic motivation, along with other subscales of motivation, should be studied further in future work with larger samples.

\section{Statistical Analyses to Study Intensity, Frequency, and Duration}

Much of the recent physical activity intensity, frequency, and duration validation literature was compiled for the current study. Many of the included studies utilized the same statistical analyses to assess agreement across measures. Spearman rank order correlation coefficients and Bland-Altman plots were the most often utilized statistical analyses for the validation studies (e.g., Dyrstad et al., 2014; Stahl \& Insana, 2014; Sternfeld et al., 2011). The ROC curve analyses conducted in the current study provide a unique analysis of the agreement of intensity, frequency, and duration of physical activity across measures. The findings from the ROC curve analyses show important discrepancies across the weeks that were not visible from the pairwise comparisons or the ICC analyses. The ROC curve analyses provide a more detailed view of the data, highlighting specific information on individual reporting behavior by analyzing the sensitivity and specificity of each data point.

\section{Limitations}

The current study significantly adds to the current literature with a focus on each of the physical activity components, as well as the use of ROC curve analyses. However, there are limitations to note. First, the sample size was relatively small, which is potentially limiting some 
robust findings. Considering the many analyses conducted and the level of significance being kept at $p<0.05$, having a larger sample size would improve the statistical power. Connectedly, the majority of the sample identified as female, curtailing the analyses on sex differences. Regarding the measures, the GLTEQ only assessed physical activity duration in 15-minute increments (Godin \& Shephard, 1997). So, per the GLTEQ, if participants engaged in less than 15 minutes of physical activity, that activity was not be included in the assessment. This could potentially be adding to the chance agreement often found between the accelerometer and GLTEQ for intensity and duration of physical activity. Additionally, although the accelerometer was treated as a "gold standard" for the study, there are equipment-based errors to consider. Some participants' accelerometers did not retain their daily physical activity intensity, frequency, and duration data. Although health coaches reminded participants to wear the accelerometer continuously except when in the water, some participants had long periods of time during which the watch was not worn. These problems all affected weekly scores. Thus, researchers need to carefully outline the importance of accelerometers to participants, as well as carefully choose the accelerometer for their study. Although certain safeguards were in place for the daily diary accounts (e.g., specified e-mail times, no backfilling; Nehrkorn et al., 2018), some participants' infrequent responding was problematic. Estimation maximation was only employed when data was available for at least four of the seven days. If participants did not have this quota, data points were not imputed. Various cut-offs were utilized (e.g., MET values, intrinsic motivation score) to assess the data. Although the current literature was carefully considered when deciding on cut-off values, there could be some flexibility in these values. These limitations should be accounted for in future studies to extend the findings and implications. 


\section{Conclusion and Future Research}

To fully and accurately study physical activity, all three physical activity components must be assessed. The results from the current study highlighted multiple pitfalls in participants' subjective accounts of intensity, frequency, and duration of physical activity across intensities. First, based on the current study's findings, participants ended with the lowest agreement when reporting activity scores and duration of sedentary behaviors, with the largest amount of inaccuracy consistently reported at Week 3. To improve agreement between measures for sedentariness, education programs that teach participants about the physical activity components and how to track each of them may be one solution. Specific to the Week 3 decrease in agreement, participants may need to be motivated or incentivized in other ways to continue accurately reporting throughout the study duration. Although the current study focused on the initial adoption of a physical activity routine, it is important to consider ways of encouraging full commitment to physical activity tracking and reporting through the extended maintenance stage. Second, besides the Week 3 moderate-to-vigorous activity score and minutes of physical activity, every activity score, frequency, and duration mean from the daily diary and GLTEQ was underestimated in comparison to accelerometer means. As mentioned, future work should focus on educating people about all intensities and duration of physical activity, while also teaching new, memorable strategies to track all components of physical activity. Third, for mild-intensity activity scores and minutes of physical activity across both weeks, the accelerometer-GLTEQ analyses resulted in the lowest agreement across the other measures' agreements. Furthermore, participants' GLTEQ accounts consistently under-reported intensity and duration of moderate-tovigorous- and mild-intensity physical activity in comparison to the accelerometer and the daily diary. Even though the GLTEQ required only one-week of recall, this extended period of time 
may be too long for participants to accurately report on these components for all intensities. Daily diaries and other short-term recall measures should be used in future studies.

Although many of the current study's findings focus on issues with reporting physical activity, there were some areas noting success in reporting behaviors. First, the daily diary accounts generally displayed the strongest agreement to the accelerometer. The close-to-themoment recall may prove to be easier for participants' tracking of their physical activity components. Thus, this is a benefit to using daily diary data to study intensity, frequency, and duration of physical activity across intensities. Second, considering the three AUC statistics that noted the strongest agreement, two of those statistics were for frequency of physical activity. Since only the accelerometer and daily diary queried participants about how often they engaged in physical activity intensities, there are fewer analyses to consider. However, this strong agreement is important to note. Future work should further explore whether participants are most successful at reporting their frequency of physical activity.

Considering age, sex, and intrinsic motivation, some group differences emerged, but mostly based on sex. As previously noted in the Discussion section, there was a small number of males included in the current study, so this is important to consider when analyzing the significant differences in the AUC scores. Across groups, however, participants generally had similar agreement across measures. A larger sample that includes a wide range of ages, more male participants, and participants with varying levels of motivation is needed in future studies to learn more about group-based reporting behaviors. Other group differences also should be further explored, as the current literature is riddled with many inconsistencies.

This study adds to and expands the study of intensity, frequency, and duration of physical activity by utilizing various measures and conducting unique, advanced statistical analyses to 
better understand the agreement across measures. With the significant inaccuracy and low agreement established in this study, participants and the general public need to better understand intensity, frequency, and duration of physical activity, as well as the various intensities, before trying to report on these components. This study brings important attention to the multifaceted nature of physical activity and demonstrates novel ways to study its three components. 


\section{References}

Adams, S. A., Matthews, C. E., Ebbeling, C. B., Moore, C. G., Cunningham, J. E., Fulton, J., \& Hebert, J. R. (2005). The effect of social desirability and social approval on self-reports of physical activity. American Journal of Epidemiology, 161(4), 389-398. doi: 10.1093/aje/kwi054

Amireault, S., \& Godin, G. (2015). The Godin-Shephard leisure-time physical activity questionnaire: Validity evidence supporting its use for classifying healthy adults into active and insufficiently active categories. Perceptual and Motor Skills, 120(2), 604-622. doi: 10.2466/03.27.PMS.120v19x7

Baltes, P. B., Lindenberger, U., \& Staudinger, U. M. (2006). Life span theory in developmental psychology. In W. Damon \& R. M. Lerner (Eds.), Handbook of child psychology: Vol. 1. Theoretical models of human development (6th ed., pp. 569-664). New York: Wiley.

Barwais, F. A., Cuddihy, T. F., \& Tomson, L. M. (2013). Physical activity, sedentary behavior and total wellness changes among sedentary adults: A 4-week randomized controlled trial. Health and Quality of Life Outcomes, 11(1), 1. doi: 10.1186/1477-7525-11-183

Bichay, A. A. F., Ramírez, J. M., Núñez, V. M., Lancho, C., Poblador, M. S., \& Lancho, J. L. (2016). Efficacy of treadmill exercises on arterial blood oxygenation, oxygen consumption and walking distance in healthy elderly people: A controlled trial. $B M C$ Geriatrics, 16(1), 110. doi: 10.1186/s12877-016-0283-5

Boletsis C., McCallum S., Landmark B.F. (2015) The Use of Smartwatches for Health Monitoring in Home-Based Dementia Care. In: Zhou J., Salvendy G. (eds) Human Aspects of IT for the Aged Population.

Boon, R. M., Hamlin, M. J., Steel, G. D., \& Ross, J. J. (2010). Validation of the New Zealand 
physical activity questionnaire (NZPAQ-LF) and the international physical activity questionnaire (IPAQ-LF) with accelerometry. British Journal of Sports Medicine, 44, 741-746. doi: 10.1136/bjsm.2008.052167

Brunes, A., Augestad, L. B., \& Gudmundsdottir, S. L. (2013). Personality, physical activity, and symptoms of anxiety and depression: the HUNT study. Social Psychiatry and Psychiatric Epidemiology, 48(5), 745-756. doi: 10.1007/s00127-012-0594-6

Buchman, A. S., Boyle, P. A., Yu, L., Shah, R. C., Wilson, R. S., \& Bennett, D. A. (2012). Total daily physical activity and the risk of AD and cognitive decline in older adults. Neurology, 78(17), 1323-1329. doi: 10.1212/WNL.0b013e3182535d35

Bull, F. C., Maslin, T. S., \& Armstrong, T. (2009). Global physical activity questionnaire (GPAQ): Nine country reliability and validity study. Journal of Physical Activity and Health, 6(6), 790-804. doi: 10.1123/jpah.6.6.790

Butte, N. F., Ekelund, U., \& Westerterp, K. R. (2012). Assessing physical activity using wearable monitors: Measures of physical activity. Medicine \& Science in Sports \& Exercise, 44(1S), S5-S12. doi: 10.1249/MSS.0b013e3182399c0e

Castillo-Retamal, M., \& Hinckson, E. A. (2011). Measuring physical activity and sedentary behaviour at work: A review. Work, 40(4), 345-357.

Centers for Disease Control and Prevention. (CDC, 2015a). How much physical activity do adults need? Atlanta: US Department of Health and Human Services. Retrieved from https://www.cdc.gov/physicalactivity/basics/adults/index.htm

Centers for Disease Control and Prevention. (CDC, 2015b). Target heart rate and estimated maximum heart rate. Atlanta: US Department of Health and Human Services. Retrieved from https://www.cdc.gov/physicalactivity/basics/measuring/heartrate.htm 
Cesari, M., Vellas, B., Hsu, F. C., Newman, A. B., Doss, H., King, A. C., ... \& Pahor, M. (2014). A physical activity intervention to treat the frailty syndrome in older persons - results from the LIFE-P study. Journals of Gerontology Series A: Biomedical Sciences and Medical Sciences, 70(2), 216-222. doi: 10.1093/gerona/glu099

Chinapaw, M. J., Slootmaker, S. M., Schuit, A. J., van Zuidam, M., \& van Mechelen, W. (2009). Reliability and validity of the Activity Questionnaire for Adults and Adolescents (AQuAA). BMC Medical Research Methodology, 9(1), 58. doi: 10.1186/1471-2288-9-58 Chodzko-Zajko, W. J., Proctor, D. N., Singh, M. A. F., Minson, C. T., Nigg, C. R., Salem, G. J., \& Skinner, J. S. (2009). Exercise and physical activity for older adults. Medicine \& Science in Sports \& Exercise, 41(7), 1510-1530. doi: 10.1249/MSS.0b013e3181a0c95c

Chou, C. H., Hwang, C. L., \& Wu, Y. T. (2012). Effect of exercise on physical function, daily living activities, and quality of life in the frail older adults: A meta-analysis. Archives of Physical Medicine and Rehabilitation, 93(2), 237-244. doi: 10.1016/j.apmr.2011.08.042

Cleland, C. L., Hunter, R. F., Kee, F., Cupples, M. E., Sallis, J. F., \& Tully, M. A. (2014). Validity of the Global Physical Activity Questionnaire (GPAQ) in assessing levels and change in moderate-vigorous physical activity and sedentary behaviour. BMC Public Health, 14(1), 1255. doi: 10.1186/1471-2458-14-1255

Cohen J. (1988). Statistical Power Analysis for the Behavioral Sciences. New York, NY: Routledge Academic.

Computer Science and Applications, Inc. (CSA; 1997). Activity Monitor Operator's Manual, Model 7164. Shalimar, FL: Computer Science and Applications.

Craig, C. L., Marshall, A. L., Sjöström, M., Bauman, A. E., Booth, M. L., Ainsworth, B. E., ... \& Oja, P. (2003). International physical activity questionnaire: 12-country reliability and 
validity. Medicine \& Science in Sports \& Exercise, 35(8), 1381-1395. doi:

10.1249/01.MSS.0000078924.61453.FB

Davies, C. A., Vandelanotte, C., Duncan, M. J., \& Van Uffelen, J. G. (2012). Associations of physical activity and screen-time on health related quality of life in adults. Preventive Medicine, 55(1), 46-49. doi: 10.1016/j.ypmed.2012.05.003

de Mello, M. T., de Aquino Lemos, V., Antunes, H. K. M., Bittencourt, L., Santos-Silva, R., \& Tufik, S. (2013). Relationship between physical activity and depression and anxiety symptoms: A population study. Journal of Affective Disorders, 149(1), 241-246. doi: 10.1016/j.jad.2013.01.035

Deci, E. L., \& Ryan, R. M. (2002). Overview of self-determination theory: An organismic dialectical perspective. Handbook of Self-Determination Research, 3-33.

Dooley, E. E., Golaszewski, N. M., \& Bartholomew, J. B. (2017). Estimating accuracy at exercise intensities: A comparative study of self-monitoring heart rate and physical activity wearable devices. JMIR mHealth and uHealth, 5(3). doi: 10.2196/mhealth.7043

Duncan, L. R., Hall, C. R., Wilson, P. M., \& Jenny, O. (2010). Exercise motivation: A crosssectional analysis examining its relationships with frequency, intensity, and duration of exercise. International Journal of Behavioral Nutrition and Physical Activity, 7(1), 7. doi: $10.1186 / 1479-5868-7-7$

Dyrstad, S. M., Hansen, B. H., Holme, I. M., \& Anderssen, S. A. (2014). Comparison of selfreported versus accelerometer-measured physical activity. Medicine \& Science in Sports \& Exercise, 46(1), 99-106. doi: 10.1249/MSS.0b013e3182a0595f

Ehrlich, C. (2012). Be careful what you wish for but also why you wish for it-Goal-striving reasons and subjective well-being. The Journal of Positive Psychology, 7(6), 493-503. 
doi: $10.1080 / 17439760.2012 .721382$

Ehrlich, C., \& Bipp, T. (2016). Goals and subjective well-being: Further evidence for goalstriving reasons as an additional level of goal analysis. Personality and Individual Differences, 89, 92-99. doi: 10.1016/j.paid.2015.10.001

Ekelund, U., Sepp, H., Brage, S., Becker, W., Jakes, R., Hennings, M., \& Wareham, N. J. (2006). Criterion-related validity of the last 7-day, short form of the International Physical Activity Questionnaire in Swedish adults. Public Health Nutrition, 9(2), 258-265. doi: 10.1079/PHN2005840

Fanning, J., Porter, G., Awick, E. A., Ehlers, D. K., Roberts, S. A., Cooke, G., ... \& McAuley, E. (2017). Replacing sedentary time with sleep, light, or moderate-to-vigorous physical activity: Effects on self-regulation and executive functioning. Journal of Behavioral Medicine, 40(2), 332-342. doi: 10.1007/s10865-016-9788-9

Fawcett, T. (2006). An introduction to ROC analysis. Pattern recognition letters, 27(8), 861-874.

Freedson, P., Bowles, H. R., Troiano, R., \& Haskell, W. (2012). Assessment of physical activity using wearable monitors: recommendations for monitor calibration and use in the field. Medicine and Science in Sports and Exercise, 44(1 Suppl 1), S1. doi: 10.1249/MSS.0b013e3182399b7e

Freund, A. M., Nikitin, J., \& Riediger, M. (2012). Successful Aging. In Weiner, Irving B. (Eds) Handbook of Psychology, Developmental Psychology (2 ${ }^{\text {nd }}$ ed). Wiley.

Friederichs, S. A., Bolman, C., Oenema, A., \& Lechner, L. (2015). Profiling physical activity motivation based on self-determination theory: a cluster analysis approach. $B M C$ Psychology, 3(1), 1. doi: 10.1186/s40359-015-0059-2

Gabriel, K. K. P., Morrow Jr, J. R., \& Woolsey, A. L. T. (2012). Framework for physical activity 
as a complex and multidimensional behavior. Journal of Physical Activity and Health, 9(s1), S11-S18. doi: 10.1123/jpah.9.s1.s11

Ghosh, D., \& Vogt, A. (2012). Outliers: An evaluation of methodologies. In Joint Statistical Meetings (pp. 3455-3460). San Diego, CA: American Statistical Association.

Gibbs, B. B., Hergenroeder, A. L., Katzmarzyk, P. T., Lee, I. M., \& Jakicic, J. M. (2015). Definition, measurement, and health risks associated with sedentary behavior. Medicine and Science in Sports and Exercise, 47(6), 1295. doi: 10.1249/MSS.0000000000000517

Godin, G., \& Shephard, R. J. (1997). Godin leisure-time exercise questionnaire. Med Sci Sports Exerc, 29(6), 36-38.

Gotsis, M., Wang, H., Spruijt-Metz, D., Jordan-Marsh, M., \& Valente, T. W. (2013). Wellness partners: Design and evaluation of a web-based physical activity diary with social gaming features for adults. JMIR Research Protocols, 2(1). doi: 10.2196/resprot.2132

Grice, H.P. (1975). Logic and conversation. In Syntax and semantics 3: Speech arts (pp.41-58). Elsevier.

Hagger, M. S., \& Chatzisarantis, N. L. (2014). An integrated behavior change model for physical activity. Exercise and Sport Sciences Reviews, 42(2), 62-69. doi:

\subsection{9/JES.0000000000000008}

Hagströmer, M., Ainsworth, B. E., Oja, P., \& Sjöström, M. (2010). Comparison of a subjective and an objective measure of physical activity in a population sample. Journal of Physical Activity and Health, 7(4), 541-550. doi: 10.1123/jpah.7.4.541

Hagströmer, M., Oja, P., \& Sjöström, M. (2006). The International Physical Activity Questionnaire (IPAQ): A study of concurrent and construct validity. Public Health Nutrition, 9(6), 755-762. doi: 10.1079/PHN2005898 
Hallal, P. C., Andersen, L. B., Bull, F. C., Guthold, R., Haskell, W., Ekelund, U., \& Lancet Physical Activity Series Working Group. (2012). Global physical activity levels: Surveillance progress, pitfalls, and prospects. The Lancet, 380(9838), 247-257. doi: 10.1016/S0140-6736(12)60646-1

Haskell, W. L., Lee, I. M., Pate, R. R., Powell, K. E., Blair, S. N., Franklin, B. A., ... \& Bauman, (2007). Physical activity and public health: Updated recommendation for adults from the American College of Sports Medicine and the American Heart Association. Circulation, 116(9), 1081. doi: 10.1249/mss.0b013e3180616b27

Hayes, A. F. (2013). Introduction to mediation, moderation, and conditional process analysis: A regression-based approach. Guilford Press.

Hekler, E. B., Buman, M. P., Haskell, W. L., Conway, T. L., Cain, K. L., Sallis, J. F., ... \& King, A. C. (2012). Reliability and validity of CHAMPS self-reported sedentary-to-vigorous intensity physical activity in older adults. Journal of Physical Activity and Health, 9(2), 225-236. doi: 10.1123/jpah.9.2.225

Hektner, J. M., Schmidt, J. A., \& Csikszentmihalyi, M. (2007). Epistemological foundations for the measurement of experience. In Experience sampling method: Measuring the quality of everyday life (chapter 1). Retrieved from https://books.google.com/books?hl=en\&lr=\&id=05e5d_KBYY0C\&oi=fnd\&pg=PR9\&dq =Experience+sampling+method:+++Measuring+the+quality+of+everyday+lif\&ots=rtES P1rj9-\&sig=d

GF9iDuTNKRvMboe034hoIl1wU\#v=onepage \&q=Experience\%20sampling\%20method $\% 3 \mathrm{~A} \% 20 \% 20 \% 20$ Measuring $\% 20$ the $\% 20$ quality $\% 20$ of $\% 20$ everyday $\% 201$ if $\& \mathrm{f}=$ false

Herrmann, S. D., Heumann, K. J., Der Ananian, C. A., \& Ainsworth, B. E. (2013). Validity and 
reliability of the global physical activity questionnaire (GPAQ). Measurement in Physical Education and Exercise Science, 17(3), 221-235. doi: 10.1080/1091367X.2013.805139

Homan, K. J., \& Tylka, T. L. (2014). Appearance-based exercise motivation moderates the relationship between exercise frequency and positive body image. Body Image, 11(2), 101-108. doi: 10.1016/j.bodyim.2014.01.003

Hoos, T., Espinoza, N., Marshall, S., \& Arredondo, E. M. (2012). Validity of the global physical activity questionnaire (GPAQ) in adult Latinas. Journal of Physical Activity and Health, 9(5), 698-705. doi: 10.1123/jpah.9.5.698

Hupin, D., Roche, F., Gremeaux, V., Chatard, J. C., Oriol, M., Gaspoz, J. M., ... \& Edouard, P. (2015). Even a low-dose of moderate-to-vigorous physical activity reduces mortality by $22 \%$ in adults aged $\geq 60$ years: A systematic review and meta-analysis. Br J Sports Med, 0, 1-8. doi: 10.1136/bjsports-2014-094306

Hutto, B., Howard, V. J., Blair, S. N., Colabianchi, N., Vena, J. E., Rhodes, D., \& Hooker, S. P. (2013). Identifying accelerometer nonwear and wear time in older adults. International Journal of Behavioral Nutrition and Physical Activity, 10(1), 120. doi: 10.1186/1479$5868-10-120$

Intel Corporation (2015). Validation of basis heart rate detection versus gold standard. Retrieved from http://docplayer.net/22998293-Validation-of-basis-heart-rate-detectionversus-gold-standard.html

Kelly, L. A., McMillan, D. G., Anderson, A., Fippinger, M., Fillerup, G., \& Rider, J. (2013). Validity of actigraphs uniaxial and triaxial accelerometers for assessment of physical activity in adults in laboratory conditions. BMC Medical Physics, 13(1), 5. doi: $10.1186 / 1756-6649-13-5$ 
Kinnunen, T. I., Tennant, P. W., McParlin, C., Poston, L., Robson, S. C., \& Bell, R. (2011). Agreement between pedometer and accelerometer in measuring physical activity in overweight and obese pregnant women. BMC Public Health, 11(1), 501. doi: $10.1186 / 1471-2458-11-501$

Kowalski, K., Rhodes, R., Naylor, P. J., Tuokko, H., \& MacDonald, S. (2012). Direct and indirect measurement of physical activity in older adults: A systematic review of the literature. International Journal of Behavioral Nutrition and Physical Activity, 9(1), 148. doi: $10.1186 / 1479-5868-9-148$

Kozey-Keadle, S., Libertine, A., Lyden, K., Staudenmayer, J., \& Freedson, P. S. (2011). Validation of wearable monitors for assessing sedentary behavior. Medicine \& Science in Sports \& Exercise, 43(8), 1561-1567. doi: 10.1249/MSS.0b013e31820ce174

Langlois, F., Vu, T. T. M., Chassé, K., Dupuis, G., Kergoat, M. J., \& Bherer, L. (2013). Benefits of physical exercise training on cognition and quality of life in frail older adults. The Journals of Gerontology: Series B, 68(3), 400-404. doi: 10.1093/geronb/gbs069

Lauderdale, M. E., Yli-Piipari, S., Irwin, C. C., \& Layne, T. E. (2015). Gender differences regarding motivation for physical activity among college students: A self-determination approach. Physical Educator, 72(5), 153-172. doi: 10.18666/TPE-2015-V72-I5-4682

Lee, P. H., Macfarlane, D. J., Lam, T. H., \& Stewart, S. M. (2011). Validity of the international physical activity questionnaire short form (IPAQ-SF): A systematic review. International Journal of Behavioral Nutrition and Physical Activity, 8(1), 115. doi: 10.1186/1479$5868-8-115$

Lee, I. M., Shiroma, E. J., Lobelo, F., Puska, P., Blair, S. N., Katzmarzyk, P. T., \& Lancet Physical Activity Series Working Group. (2012). Effect of physical inactivity on major 
non-communicable diseases worldwide: An analysis of burden of disease and life expectancy. The Lancet, 380(9838), 219-229. doi: 10.1016/S0140-6736(12)61031-9

Matthews, C. E., Chen, K. Y., Freedson, P. S., Buchowski, M. S., Beech, B. M., Pate, R. R., \& Troiano, R. P. (2008). Amount of time spent in sedentary behaviors in the United States, 2003-2004. American Journal of Epidemiology, 167(7), 875-881. doi: 10.1093/aje/kwm390

McDonnell, M. N., Hillier, S. L., Hooker, S. P., Le, A., Judd, S. E., \& Howard, V. J. (2013). Physical activity frequency and risk of incident stroke in a national US study of blacks and whites. Stroke, 44(9), 2519-2524. doi: 10.1161/STROKEAHA.113.001538

Meltzer, L. J., Hiruma, L. S., Avis, K., Montgomery-Downs, H., \& Valentin, J. (2015). Comparison of a commercial accelerometer with polysomnography and actigraphy in children and adolescents. Sleep, 38(8), 1323-1330. doi: 10.5665/sleep.4918

Miller, D. J., Freedson, P. S., \& Kline, G. M. (1994). Comparison of activity levels using the Caltrac accelerometer and five questionnaires. Medicine and Science in Sports and Exercise, 26(3), 376-382. doi: 10.1249/00005768-199403000-00016

Montgomery-Downs, H. E., Insana, S. P., \& Bond, J. A. (2012). Movement toward a novel activity monitoring device. Sleep and Breathing, 16(3), 913-917. doi: 10.1007/s11325$011-0585-y$

Moore, R., Berlowitz, D., Denehy, L., Jackson, B., \& McDonald, C. F. (2009). Comparison of pedometer and activity diary for measurement of physical activity in chronic obstructive pulmonary disease. Journal of Cardiopulmonary Rehabilitation and Prevention, 29(1), 57-61. doi: 10.1097/HCR.0b013e318192786c

Moore, S. C., Patel, A. V., Matthews, C. E., de Gonzalez, A. B., Park, Y., Katki, H. A., ... \& 
Thun, M. (2012). Leisure time physical activity of moderate to vigorous intensity and mortality: A large pooled cohort analysis. PLoS Medicine, 9(11), e1001335. doi: 10.1371/journal.pmed.1001335

Motl, R. W., \& McAuley, E. (2009). Pathways between physical activity and quality of life in adults with multiple sclerosis. Health Psychology, 28(6), 682. doi: 10.1037/a0015985

Motl, R. W., McAuley, E., \& Dlugonski, D. (2012). Reactivity in baseline accelerometer data from a physical activity behavioral intervention. Health Psychology, 31(2), 172. doi: $10.1037 / \mathrm{a} 0025965$

Motl, R. W., McAuley, E., Wynn, D., Suh, Y., \& Weikert, M. (2011). Effects of change in fatigue and depression on physical activity over time in relapsing-remitting multiple sclerosis. Psychology, Health \& Medicine, 16(1), 1-11. doi: $10.1080 / 13548506.2010 .521569$

Nelson, M. E., Rejeski, W. J., Blair, S. N., Duncan, P. W., Judge, J. O., King, A. C., ... \& Castaneda-Sceppa, C. (2007). Physical activity and public health in older adults: Recommendation from the American College of Sports Medicine and the American Heart Association. Circulation, 116(9), 1094. doi: 10.1249/mss.0b013e3180616aa2

Netz, Y., Wu, M. J., Becker, B. J., \& Tenenbaum, G. (2005). Physical activity and psychological well-being in advanced age: A meta-analysis of intervention studies. Psychology and Aging, 20(2), 272-284. Doi: 10.1037/0882-7974.20.2.272

Ortlieb, S., Dias, A., Gorzelniak, L., Nowak, D., Karrasch, S., Peters, A., ... \& Schulz, H. (2014). Exploring patterns of accelerometry-assessed physical activity in elderly people. International Journal of Behavioral Nutrition and Physical Activity, 11, 28. doi: $10.1186 / 1479-5868-11-28$ 
Oshima, T., Berger, M. M., De Waele, E., Guttormsen, A. B., Heidegger, C. P., Hiesmayr, M., ... \& Pichard, C. (2017). Indirect calorimetry in nutritional therapy. A position paper by the ICALIC study group. Clinical Nutrition, 36(3), 651-662. doi: 10.1016/j.clnu.2016.06.010

Prince, S. A., Adamo, K. B., Hamel, M. E., Hardt, J., Gorber, S. C., \& Tremblay, M. (2008). A comparison of direct versus self-report measures for assessing physical activity in adults: A systematic review. International Journal of Behavioral Nutrition and Physical Activity, 5(1), 56. doi: 10.1186/1479-5868-5-56

Ruffault, A., Bernier, M., Juge, N., \& Fournier, J. F. (2016). Mindfulness may moderate the relationship between intrinsic motivation and physical activity: A cross-sectional study. Mindfulness, 7(2), 445-452. doi: 10.1007/s12671-015-0467-7

Sabia, S., van Hees, V. T., Shipley, M. J., Trenell, M. I., Hagger-Johnson, G., Elbaz, A., ... \& Singh-Manoux, A. (2014). Association between questionnaire-and accelerometerassessed physical activity: The role of sociodemographic factors. American Journal of Epidemiology, 179(6), 781-790. doi: 10.1093/aje/kwt330

Sallis, J. F., \& Saelens, B. E. (2000). Assessment of physical activity by self-report: Status, limitations, and future directions. Research Quarterly for Exercise and Sport, 71(sup2), 1-14. doi: 10.1080/02701367.2000.11082780

Santos, D. A., Silva, A. M., Baptista, F., Santos, R., Vale, S., Mota, J., \& Sardinha, L. B. (2012). Sedentary behavior and physical activity are independently related to functional fitness in older adults. Experimental Gerontology, 47(12), 908-912. doi:10.1016/j.exger.2012.07.011

Schaie, K. W. (2010). Historical Influences on Aging and Behavior. Handbook of the Psychology of Aging, 7e, 41. doi: 10.1016/B978-0-12-380882-0.00003-6 
Schoenborn, C. A., Adams, P. F., \& Peregoy, J. A. (2013). Health behaviors of adults: United States, 2008-2010. Vital and health statistics. Series 10, Data from the National Health Survey, (257), 1-184.

Schoonjans, F. (2017). MedCalc manual. MedCalc Software bvba.

Schwarz, N. (1999). Self-reports: How the questions shape the answers. American Psychologist, 54, 93-105. doi: 10.1037/0003-066X.54.2.93

Sirard, J. R., Hannan, P., Cutler, G. J., \& Nuemark-Sztainer, D. (2013). Evaluation of 2 selfreport measures of physical activity with accelerometry in young adults. Journal of Physical Activity and Health, 10(1), 85-96. doi: 10.1123/jpah.10.1.85

Slootmaker, S. M., Schuit, A. J., Chinapaw, M. J., Seidell, J. C., \& Van Mechelen, W. (2009). Disagreement in physical activity assessed by accelerometer and self-report in subgroups of age, gender, education and weight status. International Journal of Behavioral Nutrition and Physical Activity, 6(1), 17. doi: 10.1186/1479-5868-6-17

Spindler, K. L. G., Pallisgaard, N., Appelt, A. L., Andersen, R. F., Schou, J. V., Nielsen, D., ... \& Jakobsen, A. (2015). Clinical utility of KRAS status in circulating plasma DNA compared to archival tumour tissue from patients with metastatic colorectal cancer treated with anti-epidermal growth factor receptor therapy. European Journal of Cancer, 51(17), 2678-2685. doi: 10.1016/j.ejca.2015.06.118

Stahl, S. T., \& Insana, S. P. (2014). Caloric expenditure assessment among older adults: Criterion validity of a novel accelerometry device. Journal of Health Psychology, 19(11), 1382-1387. doi: 10.1177/1359105313490771

Staudenmayer, J., Pober, D., Crouter, S., Bassett, D., \& Freedson, P. (2009). An artificial neural network to estimate physical activity energy expenditure and identify physical activity 
type from an accelerometer. Journal of Applied Physiology, 107(4), 1300-1307. doi:

10.1152/japplphysiol.00465.2009

Stel, V. S., Smit, J. H., Pluijm, S. M., Visser, M., Deeg, D. J., \& Lips, P. (2004). Comparison of the LASA Physical Activity Questionnaire with a 7-day diary and pedometer. Journal of Clinical Epidemiology, 57(3), 252-258. doi: 10.1016/j.jclinepi.2003.07.008

Sternfeld, B., Jiang, S. F., Picchi, T., Chasan-Taber, L., Ainsworth, B., \& Quesenberry Jr, C. P. (2012). Evaluation of a cell phone-based physical activity diary. Medicine and Science in Sports and Exercise, 44(3), 487-495. doi: 10.1249/MSS.0b013e3182325f45

Stewart, A. L., Mills, K. M., King, A. C., Haskell, W. L., Gillis, D. A. W. N., \& Ritter, P. L. (2001). CHAMPS physical activity questionnaire for older adults: Outcomes for interventions. Medicine \& Science in Sports \& Exercise, 33(7), 1126-1141. doi: $10.1097 / 00005768-200107000-00010$

Strath, S. J., Kaminsky, L. A., Ainsworth, B. E., Ekelund, U., Freedson, P. S., Gary, R. A., ... \& Swartz, A. M. (2013). Guide to the assessment of physical activity: Clinical and research applications. Circulation, 128(20), 2259-2279. doi: 10.1161/01.cir.0000435708.67487.da

Sylvia, L. G., Bernstein, E. E., Hubbard, J. L., Keating, L., \& Anderson, E. J. (2014). A practical guide to measuring physical activity. Journal of the Academy of Nutrition and Dietetics, 114(2), 199. doi: 10.1016/j.jand.2013.09.018

Taylor-Piliae, R. E., Fair, J. M., Haskell, W. L., Varady, A. N., Iribarren, C., Hlatky, M. A., ... \& Fortmann, S. P. (2010). Validation of the Stanford Brief Activity Survey: Examining psychological factors and physical activity levels in older adults. Journal of Physical Activity and Health, 7(1), 87-94. doi: 10.1123/jpah.7.1.87

Teixeira, P. J., Carraça, E. V., Markland, D., Silva, M. N., \& Ryan, R. M. (2012). Exercise, 
physical activity, and self-determination theory: A systematic review. International Journal of Behavioral Nutrition and Physical Activity, 9(1), 78. doi: 0.1186/1479-5868-978

Troiano, R. P., Berrigan, D., Dodd, K. W., Masse, L. C., Tilert, T., \& McDowell, M. (2007). Physical activity in the United States measured by accelerometer. Medicine and Science in Sports and Exercise, 40(1), 181. doi: 10.1249/mss.0b013e31815a51b3

Tucker, J. M., Welk, G. J., \& Beyler, N. K. (2011). Physical activity in US adults: Compliance with the physical activity guidelines for Americans. American Journal of Preventive Medicine, 40(4), 454-461.

Tudor-Locke, C. E., \& Myers, A. M. (2001). Challenges and opportunities for measuring physical activity in sedentary adults. Sports Medicine, 31(2), 91-100. doi: $10.2165 / 00007256-200131020-00002$

United States Department of Health and Human Services (HHS; 2008). 2008 physical activity guidelines for Americans. Washington, DC: United States Department of Health and Human Services, Centers for Disease Control and Prevention.

United States Department of Health and Human Services (HHS; 2017). Physical inactivity. Washington, DC: United States Department of Health and Human Services, Centers for Disease Control and Prevention.

United States Department of Health and Human Services (HHS; 2019). Physical activity guidelines for Americans. Washington, DC: United States Department of Health and Human Services, Centers for Disease Control and Prevention.

Vancampfort, D., De Hert, M., Vansteenkiste, M., De Herdt, A., Scheewe, T. W., Soundy, A., ... \& Probst, M. (2013). The importance of self-determined motivation towards physical 
activity in patients with schizophrenia. Psychiatry Research, 210(3), 812-818. doi:

10.1016/j.psychres.2013.10.004

Van Holle, V., De Bourdeaudhuij, I., Deforche, B., Van Cauwenberg, J., \& Van Dyck, D. (2015). Assessment of physical activity in older Belgian adults: validity and reliability of an adapted interview version of the long International Physical Activity Questionnaire (IPAQ-L). BMC Public Health, 15(1), 433. doi: 10.1186/s12889-015-1785-3

Vandelanotte, C., De Bourdeaudhuij, I., Philippaerts, R., Sjöström, M., \& Sallis, J. (2005).

Reliability and validity of a computerized and Dutch version of the International Physical Activity Questionnaire (IPAQ). Journal of Physical Activity and Health, 2(1), 63-75. doi: 10.1123/jpah.2.1.63

Vavra, K. L., Saadeh, C. E., Rosen, A. L., Uptigrove, C. E., \& Srkalovic, G. (2013). Improving the relative dose intensity of systemic chemotherapy in a community-based outpatient cancer center. Journal of Oncology Practice, 9(5), e203-e211.

Verdelho, A., Madureira, S., Ferro, J. M., Baezner, H., Blahak, C., Poggesi, A., ... \& Waldemar, G. (2012). Physical activity prevents progression for cognitive impairment and vascular dementia. Stroke, 43(12), 3331-3335.

Warburton, D. E., Nicol, C. W., \& Bredin, S. S. (2006). Health benefits of physical activity: The evidence. Canadian Medical Association Journal, 174(6), 801-809. doi: 10.1503/cmaj.051351

Warren, J. M., Ekelund, U., Besson, H., Mezzani, A., Geladas, N., \& Vanhees, L. (2010). Assessment of physical activity-a review of methodologies with reference to epidemiological research: A report of the exercise physiology section of the European Association of Cardiovascular Prevention and Rehabilitation. European Journal of 
Cardiovascular Prevention \& Rehabilitation, 17(2), 127-139.

Washburn, R. A., Smith, K. W., Jette, A. M., \& Janney, C. A. (1993). The Physical Activity Scale for the Elderly (PASE): Development and evaluation. Journal of Clinical Epidemiology, 46(2), 153-162. doi: 10.1016/0895-4356(93)90053-4

Watkinson, C., van Sluijs, E. M., Sutton, S., Hardeman, W., Corder, K., \& Griffin, S. J. (2010). Overestimation of physical activity level is associated with lower BMI: A cross-sectional analysis. International Journal of Behavioral Nutrition and Physical Activity, 7(1), 68. doi: $10.1186 / 1479-5868-7-68$

Wen, C. P., Wai, J. P. M., Tsai, M. K., Yang, Y. C., Cheng, T. Y. D., Lee, M. C., ... \& Wu, X. (2011). Minimum amount of physical activity for reduced mortality and extended life expectancy: A prospective cohort study. The Lancet, 378(9798), 1244-1253. doi: $10.1016 / \mathrm{S} 0140-6736(11) 60749-6$

Westerterp, K. R. (2009). Assessment of physical activity: A critical appraisal. European Journal of Applied Physiology, 105(6), 823-828. doi: 10.1007/s00421-009-1000-2

Wichers, M., Peeters, F., Rutten, B. P., Jacobs, N., Derom, C., Thiery, E., ... \& van Os, J. (2012). A time-lagged momentary assessment study on daily life physical activity and affect. Health Psychology, 31(2), 135. 10.1037/a0025688

World Health Organization. (WHO, 2017a). Global strategy on diet, physical activity and health: Physical activity. Retrieved from http://www.who.int/dietphysicalactivity/pa/en/ World Health Organization. (WHO, 2017b). Global strategy on diet, physical activity and health: What is moderate-intensity and vigorous-intensity physical activity? Retrieved from http://www.who.int/dietphysicalactivity/physical_activity_intensity/en/

World Health Organization. (WHO, 2019). Global strategy on diet, physical activity and health: 
Physical Activity and Adults. Retrieved from

https://www.who.int/dietphysicalactivity/factsheet_adults/en/

Zhu, W., Wadley, V. G., Howard, V. J., Hutto, B., Blair, S. N., \& Hooker, S. P. (2017).

Objectively measured physical activity and cognitive function in older

adults. Medicine and Science in Sports and Exercise, 49(1), 47-53. doi:

10.1249/MSS.0000000000001079

Zou, K. H., O’Malley, A. J., \& Mauri, L. (2007). Receiver-operating characteristic analysis for evaluating diagnostic tests and predictive models. Circulation, 115(5), 654-657. doi:

10.1161/CIRCULATIONAHA.105.594929 
Table 1.

Physical activity intensity, duration, and frequency findings from validation studies.

\begin{tabular}{llllll}
\hline & Vigorous & $\begin{array}{l}\text { Moderate-to- } \\
\text { Vigorous }\end{array}$ & Moderate & Mild & Sedentary \\
\hline Intensity & $\rho=.40-.45$ & $\rho=.40$ & $\begin{array}{l}\text { ns; } \rho=.40 \text {; lack } \\
\text { of data }\end{array}$ & ns; lack of data & $\rho<.30$ \\
Duration & $\rho=.23-.79$ & $\begin{array}{l}\rho=.23-.79 \text { in } \\
\text { general; } \rho=-.02-.26 \\
\text { for specific activities }\end{array}$ & ns; $\rho=-.02-.79$ & $\begin{array}{l}\text { ns; } \rho=.27 \text { in } \\
\text { general, } \rho=.09-\end{array}$ & $\begin{array}{l}\text { ns; } \rho=.12- \\
.21 \text { specific } \\
\text { Frequency }\end{array}$ \\
& $\rho=.42-.51$ & $\begin{array}{l}\rho=.27-.38 \text { in } \\
\text { general; } \rho=.08-.29 \\
\text { for specific activities }\end{array}$ & Lack of data & $\rho=.33$ & $\begin{array}{l}\text { Lack of } \\
\text { data }\end{array}$ \\
& & & & \\
\hline
\end{tabular}


Table 2.

Mean activity scores (intensity)

\begin{tabular}{lc}
\hline & Mean (SD) \\
\hline Accelerometer & \\
Week 2 Moderate-to-Vigorous & $1,644.11(1,337.60)$ \\
Week 2 Mild & $6,158.92(2,056.72)$ \\
Week 2 Sedentary & $3,710.17(1,302.01)$ \\
Week 3 Moderate-to-Vigorous & $1,557.75(1,264.08)$ \\
Week 3 Mild & $6,094.07(2,284.24)$ \\
Week 3 Sedentary & $3,651.83(1,341.77)$ \\
GLTEQ & \\
Week 2 Moderate-to-Vigorous & $1,209.81(1,170.94)$ \\
Week 2 Mild & $570.20(1,006.05)$ \\
Week 3 Moderate-to-Vigorous & $1,101.67(728.85)$ \\
Week 3 Mild & $627.00(839.44)$ \\
Daily Diary & \\
Week 2 Moderate-to-Vigorous & $1,547.85(1,978.04)$ \\
Week 2 Mild & $1,890.34(1,863.86)$ \\
Week 2 Sedentary & $1,155.71(1,103.95)$ \\
Week 3 Moderate-to-Vigorous & $2,082.80(2,699.31)$ \\
Week 3 Mild & $2,217.12(2,534.77)$ \\
Week 3 Sedentary & $1,405.04(1,114.44)$ \\
\hline
\end{tabular}

Note. Activity scores for intensity were generated by multiplying the minutes (e.g., duration) by the appropriate MET value for each intensity. 
Table 3 .

Mean number of days (frequency) engaged in physical activity.

\begin{tabular}{ll}
\hline & Mean (SD) \\
\hline Accelerometer & \\
Week 2 Moderate-to-Vigorous & $4.69(2.18)$ \\
Week 2 Mild & $6.65(1.18)$ \\
Week 2 Sedentary & $6.63(1.17)$ \\
Week 3 Moderate-to-Vigorous & $4.55(1.94)$ \\
Week 3 Mild & $6.45(1.46)$ \\
Week 3 Sedentary & $6.45(1.51)$ \\
Daily Diary & \\
Week 2 Moderate-to-Vigorous & $3.27(2.51)$ \\
Week 2 Mild & $4.27(2.61)$ \\
Week 2 Sedentary & $3.27(2.51)$ \\
Week 3 Moderate-to-Vigorous & $3.42(2.60)$ \\
Week 3 Mild & $4.63(2.70)$ \\
Week 3 Sedentary & $4.44(2.81)$ \\
\hline
\end{tabular}


Table 4.

Mean number of minutes (duration) engaged in physical activity.

\begin{tabular}{lc}
\hline & Mean (SD) \\
\hline Accelerometer & $274.02(222.93)$ \\
Week 2 Moderate-to-Vigorous & $1,866.34(623.25)$ \\
Week 2 Mild & $3,403.83(1,194.50)$ \\
Week 2 Sedentary & $259.63(210.68)$ \\
Week 3 Moderate-to-Vigorous & $1,846.69(692.19)$ \\
Week 3 Mild & $3,350.30(1,230.99)$ \\
Week 3 Sedentary & \\
GLTEQ & $201.63(195.16)$ \\
Week 2 Moderate-to-Vigorous & $172.79(304.86)$ \\
Week 2 Mild & $183.61(121.47)$ \\
Week 3 Moderate-to-Vigorous & $190.00(254.37)$ \\
Week 3 Mild & \\
Daily Diary & $257.98(329.67)$ \\
Week 2 Moderate-to-Vigorous & $572.83(564.80)$ \\
Week 2 Mild & $1,240.71(1209.79)$ \\
Week 2 Sedentary & $347.13(449.88)$ \\
Week 3 Moderate-to-Vigorous & $671.85(768.11)$ \\
Week 3 Mild & $1,235.19(994.55)$ \\
Week 3 Sedentary &
\end{tabular}


Table 5.

Intraclass correlations for the three measures and intensities.

\begin{tabular}{|c|c|}
\hline & $\mathrm{ICC}(95 \% \mathrm{CI})$ \\
\hline \multicolumn{2}{|l|}{ Intensity } \\
\hline Week 2 Moderate-to-Vigorous (accelerometer and GLTEQ) & $-0.132(-0.396,0.152)$ \\
\hline Week 2 Moderate-to-Vigorous (accelerometer and daily diary) & $0.094(-0.217,0.387)$ \\
\hline Week 2 Moderate-to-Vigorous (GLTEQ and daily diary) & $-0.076(-0.372,0.234)$ \\
\hline Week 2 Mild (accelerometer and GLTEQ) & $0.217(-0.066,0.467)$ \\
\hline Week 2 Mild (accelerometer and daily diary) & $-0.011(-0.318,0.298)$ \\
\hline Week 2 Mild (GLTEQ and daily diary) & $-0.009(-0.313,0.296)$ \\
\hline Week 2 Sedentary (accelerometer and daily diary) & $0.104(-0.203,0.393)$ \\
\hline Week 3 Moderate-to-Vigorous (accelerometer and GLTEQ) & $-0.157(-0.420,0.130)$ \\
\hline Week 3 Moderate-to-Vigorous (accelerometer and daily diary) & $0.220(-0.108,0.505)$ \\
\hline Week 3 Moderate-to-Vigorous (GLTEQ and daily diary) & $-0.033(-0.334,0.274)$ \\
\hline Week 3 Mild (accelerometer and GLTEQ) & $0.049(-0.235,0.326)$ \\
\hline Week 3 Mild (accelerometer and daily diary) & $0.186(-0.143,0.478)$ \\
\hline Week 3 Mild (GLTEQ and daily diary) & $0.216(-0.94,0.489)$ \\
\hline Week 3 Sedentary (accelerometer and daily diary) & $-0.276(-0.551,0.054)$ \\
\hline \multicolumn{2}{|l|}{ Frequency } \\
\hline Week 2 Moderate-to-Vigorous (accelerometer and daily diary) & $0.114(-0.193,0.401)$ \\
\hline Week 2 Mild (accelerometer and daily diary) & $0.285(-0.018,0.539)$ \\
\hline Week 2 Sedentary (accelerometer and daily diary) & $0.227(-0.079,0.494)$ \\
\hline Week 3 Moderate-to-Vigorous (accelerometer and daily diary) & $0.152(-0.186,0.458)$ \\
\hline Week 3 Mild (accelerometer and daily diary) & $0.008(-0.309,0.323)$ \\
\hline Week 3 Sedentary (accelerometer and daily diary) & $0.014(-0.308,0.333)$ \\
\hline \multicolumn{2}{|l|}{ Duration } \\
\hline Week 2 Moderate-to-Vigorous (accelerometer and GLTEQ) & $-0.132(-0.396,0.152)$ \\
\hline Week 2 Moderate-to-Vigorous (accelerometer and daily diary) & $0.094(-0.217,0.387)$ \\
\hline Week 2 Moderate-to-Vigorous (GLTEQ and daily diary) & $-0.076(-0.372,0.234)$ \\
\hline Week 2 Mild (accelerometer and GLTEQ) & $-0.217(-0.066,0.467)$ \\
\hline Week 2 Mild (accelerometer and daily diary) & $-0.011(-0.318,0.298)$ \\
\hline Week 2 Mild (GLTEQ and daily diary) & $-0.009(-0.313,0.296)$ \\
\hline Week 2 Sedentary (accelerometer and daily diary) & $0.122(-0.185,0.408)$ \\
\hline Week 3 Moderate-to-Vigorous (accelerometer and GLTEQ) & $-0.157(-0.420,0.130)$ \\
\hline Week 3 Moderate-to-Vigorous (accelerometer and daily diary) & $0.220(-0.108,0.505)$ \\
\hline Week 3 Moderate-to-Vigorous (GLTEQ and daily diary) & $-0.033(-0.334,0.274)$ \\
\hline Week 3 Mild (accelerometer and GLTEQ) & $0.049(-0.235,0.326)$ \\
\hline Week 3 Mild (accelerometer and daily diary) & $0.183(-0.146,0.476)$ \\
\hline Week 3 Mild (GLTEQ and daily diary) & $0.216(-0.094,0.489)$ \\
\hline Week 3 Sedentary (accelerometer and daily diary) & $-0.237(-0.518,0.090)$ \\
\hline
\end{tabular}


Table 6.

ROC curve analysis AUC statistics for activity scores (intensity). Accelerometer and Accelerometer and GLTEQ Daily Diary AUC $(95 \%$ CI) AUC $(95 \%$ CI)

Daily Diary and GLTEQ

AUC $(95 \%$ CI)

Week 2

Moderate-to-vigorous

Mild

$0.596(0.413,0.778)$

$0.539(0.348,0.730)$

AUC (95\% CI)

Sedentary

$0.484(0.247,0.722)$

$0.671(0.429,0.914)$

$0.535(0.352,0.718)$

Week 3

$0.509(0.315,0.703)$

Moderate-to-vigorous

Mild

$0.514(0.335,0.693)$

$0.447(0.272,0.623)$

$0.617(0.421,0.814)$

$0.573(0.330,0.816)$

$.592(0.407,0.776)$

Sedentary

$0.331(0.136,0.526)$

$0.794(0.646,0.942)$

- $\quad 0.331(0.136,0.526)$


Table 7.

$\underline{\text { ROC curve analysis AUC statistics for frequency. }}$ Accelerometer and Daily Diary

AUC $(95 \% \mathrm{CI})$

\begin{tabular}{ll}
\hline Week 2 & \\
Moderate-to-vigorous & $0.480(0.238,0.721)$ \\
Mild & $0.836(0.688,0.983)$ \\
Sedentary & $0.686(0.389,0.984)$ \\
Week 3 & \\
Moderate-to-vigorous & $0.603(0.322,0.885)$ \\
Mild & $0.546(0.339,0.753)$ \\
Sedentary & $0.583(0.333,0.833)$ \\
\hline
\end{tabular}


Table 8.

ROC curve analysis AUC statistics for duration. Accelerometer and GLTEQ Accelerometer and Daily Diary and AUC $(95 \% \mathrm{CI})$ Daily Diary AUC (95\% CI) GLTEQ

Week 2

Moderate-to-vigorous

Mild

$0.598(0.418,0.779)$

$0.539(0.348,0.730)$

AUC $(95 \% \mathrm{CI})$

Sedentary

$0.484(0.247,0.722)$

$0.671(0.429,0.914)$

$0.537(0.356,0.719)$

Week 3

$0.537(0.340,0.734)$

Moderate-to-vigorous

Mild

$0.514(0.335,0.693)$

$0.617(0.421,0.814)$

$0.592(0.407,0.776)$

Sedentary

$0.447(0.272,0.623)$

$0.573(0.330,0.816)$

$0.794(0.646,0.942)$ 
Table 9.

ROC curve analysis AUC statistics for age.

Accelerometer and

GLTEQ

Group 1 AUC $(95 \%$ CI)

Group 2 AUC $(95 \%$ CI)
Accelerometer and

Daily Diary

Group 1 AUC (95\% CI)

Group 2 AUC $(95 \%$ CI)
Daily Diary and GLTEQ Group 1 AUC $(95 \%$ CI)

Group 2 AUC $(95 \% \mathrm{CI})$

Intensity

Week 2

Moderate-to-vigorous

$0.662(0.180,0.887)$

$0.546(0.113,0.804)$

$0.725(0.124,0.937)$

$0.571(0.300,0.756)$

$0.536(0.253,0.734)$

$0.459(0.203,0.656)$

$0.613(0.117,0.864)$

$0.591(0.229,0.809)$

$0.448(0.271,0.596)$

$0.880(0.664,0.961)$

$0.660(0.274,0.863)$

$0.644(0.316,0.834)$

Sedentary

$0.404(0.108,0.635)$

Week 3

Moderate-to-vigorous

$0.700(0.313,0.888)$

$0.375(-0.053,0.687)$

$0.681(0.190,0.899)$

$0.434(0.190,0.628)$

$0.510(0.149,0.752)$

$0.834(0.550,0.945)$

Mild

$0.606(0.227,0.825)$

$0.403(-0.079,0.732)$

$0.561(0.156,0.804)$

$0.369(0.136,0.564)$

$0.713(0.313,0.898)$

$0.375(-0.178,0.748)$

$0.289(0.032,0.510)$

Frequency

Week 2

Moderate-to-vigorous

$-$

$0.300(0.008,0.545)$

$0.567(0.224,0.785)$

Mild

Sedentary

Week 3

Moderate-to-vigorous

Mild

Sedentary

Duration

Week 2

Moderate-to-vigorous

$0.662(0.180,0.887)$

$0.577(0.311,0.759)$

0.467 (0.170, 0.686)

$0.818(0.515,0.940)$

$0.367(0.150,0.550)$

$0.515(-0.088,0.841)$

Mild

$0.473(0.010,0.769)$

0.000

$0.292(-0.128,0.618)$

$0.500(-0.300,0.887)$

$0.600(0.233,0.817)$

$0.667(0.104,0.906)$

$0.415(0.076,0.667)$

Sedentary

$0.546(0.113,0.804)$

$0.536(0.253,0.734)$

$0.613(0.117,0.864)$

$0.880(0.664,0.961)$

$0.690(0.300,0.882)$

$0.426(0.116,0.660)$

\section{Week 3}


Table 9 Continued

$\begin{array}{lclc}\text { Moderate-to-vigorous } & 0.700(0.313,0.888) & 0.375(-0.053,0.687) & 0.681(0.190,0.899) \\ & 0.434(0.190,0.628) & 0.510(0.149,0.752) & 0.834(0.550,0.945) \\ \text { Mild } & 0.606(0.227,0.825) & 0.403(-0.079,0.732) & 0.561(0.156,0.804) \\ & 0.369(0.136,0.564) & 0.713(0.313,0.898) & 0.891(0.603,0.974) \\ \text { Sedentary } & - & 0.400(-0.126,0.750) & - \\ & & 0.360(0.085,0.584) & \end{array}$

Note. AUC statistics in bold note a significant group difference. 
Table 10.

ROC curve analysis AUC statistics for sex.

\begin{tabular}{|c|c|c|c|}
\hline & $\begin{array}{l}\text { Accelerometer and } \\
\text { GLTEQ } \\
\text { Group } 1 \text { AUC }(95 \% \mathrm{CI}) \\
\text { Group } 2 \text { AUC }(95 \% \mathrm{CI})\end{array}$ & $\begin{array}{l}\text { Accelerometer and } \\
\text { Daily Diary } \\
\text { Group } 1 \text { AUC }(95 \% \mathrm{CI}) \\
\text { Group } 2 \text { AUC }(95 \% \mathrm{CI})\end{array}$ & $\begin{array}{l}\text { Daily Diary and GLTEQ } \\
\text { Group } 1 \text { AUC }(95 \% \mathrm{CI}) \\
\text { Group } 2 \text { AUC }(95 \% \mathrm{CI})\end{array}$ \\
\hline \multicolumn{4}{|l|}{ Intensity } \\
\hline Moderate-to-vigorous & $\begin{array}{l}0.551(0.315,0.723) \\
0.688(-0.351,0.968)\end{array}$ & $\begin{array}{l}0.553(0.293,0.736) \\
0.575(-0.434,0.944)\end{array}$ & $\begin{array}{l}0.548(0.305,0.734) \\
0.550(-0.055,0.860)\end{array}$ \\
\hline Mild & $\begin{array}{l}0.550(0.283,0.738) \\
0.056(-0.054,0.163)\end{array}$ & $\begin{array}{l}0.624(0.057,0.886) \\
0.750(-0.221,0.974)\end{array}$ & $\begin{array}{l}0.657(0.368,0.831) \\
0.542(0.008,0.835)\end{array}$ \\
\hline Sedentary & - & $\begin{array}{l}0.439(0.197,0.630) \\
0.889(0.171,0.990)\end{array}$ & - \\
\hline \multicolumn{4}{|l|}{ Week 3} \\
\hline Moderate-to-vigorous & $\begin{array}{l}0.471(0.248,0.646) \\
0.643(-0.306,0.951)\end{array}$ & $\begin{array}{l}0.452(0.152,0.676) \\
0.400(-0.147,0.760)\end{array}$ & $\begin{array}{l}0.867(0.630,0.956) \\
0.450(-0.102,0.790)\end{array}$ \\
\hline Mild & $\begin{array}{l}0.414(0.207,0.586) \\
0.000\end{array}$ & $\begin{array}{l}0.548(0.218,0.765) \\
0.000\end{array}$ & $\begin{array}{l}0.826(0.541,0.941) \\
0.722(0.161,0.931)\end{array}$ \\
\hline Sedentary & - & $\begin{array}{l}0.374(0.130,0.575) \\
0.000\end{array}$ & - \\
\hline \multicolumn{4}{|l|}{ Frequency } \\
\hline Moderate-to-vigorous & - & $\begin{array}{l}0.534(0.280,0.718) \\
0.056(-0.054,0.163)\end{array}$ & - \\
\hline Mild & - & $\begin{array}{l}0.799(0.491,0.930) \\
0.611(0.201,0.839)\end{array}$ & - \\
\hline Sedentary & - & $\begin{array}{l}0.517(-0.100,0.847) \\
0.389(0.090,0.624)\end{array}$ & - \\
\hline \multicolumn{4}{|l|}{ Week 3} \\
\hline Moderate-to-vigorous & - & $\begin{array}{l}0.352(-0.071,0.668) \\
0.000\end{array}$ & - \\
\hline Mild & - & $\begin{array}{l}0.526(0.250,0.723) \\
0.000\end{array}$ & - \\
\hline Sedentary & - & $\begin{array}{l}0.500(0.223,0702) \\
0.000\end{array}$ & - \\
\hline \multicolumn{4}{|l|}{ Duration } \\
\hline Moderate-to-vigorous & $\begin{array}{c}0.553(0.317,0.724) \\
0.688(-0.351,0.968)\end{array}$ & $\begin{array}{l}0.553(0.293,0.736) \\
0.575(-0.434,0.944)\end{array}$ & $\begin{array}{c}0.548(0.305,0.724) \\
0.575(-0.013,0.868)\end{array}$ \\
\hline Mild & $\begin{array}{c}0.550(0.283,0.738) \\
0.056(-0.054,0.163)\end{array}$ & $\begin{array}{l}0.624(0.057,0.886) \\
0.750(-0.221,0.974)\end{array}$ & $\begin{array}{l}0.657(0.368,0.831) \\
0.542(0.008,0.835)\end{array}$ \\
\hline Sedentary & - & $\begin{array}{l}0.465(0.214,0.658) \\
1.000\end{array}$ & - \\
\hline
\end{tabular}

Week 3 
Table 10 Continued

Moderate-to-vigorous

Mild

Sedentary
$0.471(0.248,0.646) \quad 0.452(0.152,0.676)$ $0.643(-0.306,0.951) \quad 0.400(-0.147,0.760)$ $0.414(0.207,0.586) \quad 0.548(0.218,0.765)$

0.000
0.000

$0.402(0.156,0.601)$ 0.000
$0.867(0.630,0.956)$

$0.450(-0.102,0.790)$

$0.826(0.541,0.941)$

$0.722(0.161,0.931)$

Note. AUC statistics in bold note a significant group difference. 
Table 11.

ROC curve analysis AUC statistics for intrinsic motivation.

Accelerometer and Accelerometer and

GLTEQ

Group 1 AUC $(95 \%$ CI)

Group 2 AUC $(95 \%$ CI)
Daily Diary

Group 1 AUC (95\% CI)

Group 2 AUC $(95 \%$ CI)

\section{Intensity \\ Week 2 \\ Moderate-to-vigorous \\ Mild

$$
\begin{aligned}
& 0.592(0.217,0.815) \\
& 0.618(0.285,0.818) \\
& 0.463(0.128,0.703) \\
& 0.375(0.195,0.531)
\end{aligned}
$$

Sedentary

Week 3

Moderate-to-vigorous

Mild

Sedentary

Frequency

Week 2

Moderate-to-vigorous

Mild

Sedentary

Week 3

Moderate-to-vigorous

Mild

Sedentary

Duration

Week 2

Moderate-to-vigorous

Mild

Sedentary

Week 3

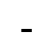

$$
\begin{aligned}
& 0.543(0.145,0.790) \\
& 0.460(0.182,0.671) \\
& 0.518(0.139,0.764) \\
& 0.338(0.090,0.547)
\end{aligned}
$$

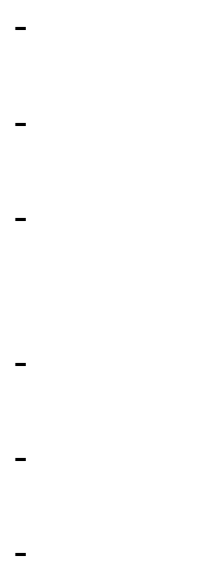

$0.447(0.150,0.669)$

$0.352(-0.072,0.667)$

$0.833(0.240,0.973)$

$0.765(0.370,0.926)$

$0.472(-0.441,0.905)$

$0.347(0.015,0.610)$

$0.500(-0.586,0.944)$

$0.167(-0.058,0.376)$

$0.656(-0.0 .78,0.929)$

$0.500(0.157,0.736)$

$0.250(0.038,0.441)$

$0.639(0.272,0.844)$

$0.597(0.227,0.816)$

$0.618(0.285,0.818)$

$0.463(0.128,0.703)$

$0.375(0.195,0.531)$
$0.536(0.077,0.807)$

$0.541(0.215,0.758)$

$0.808(0.330,0.956)$

$$
0.000
$$

$0.700(0.242,0.903)$

$0.429(0.076,0.686)$
Daily Diary and GLTEQ Group 1 AUC $(95 \%$ CI)

Group 2 AUC $(95 \%$ CI)

\section{$0.527(0.148,0.771)$}

$0.688(0.334,0.871)$

$0.843(0.484,0.959)$

$0.779(0.469,0.918)$

$0.764(0.209,0.947)$
$0.599(0.249,0.810)$

$0.385(0.106,0.608)$

$0.643(0.221,0.862)$

$0.657(0.388,0.823)$
$0.599(0.249,0.810)$

$0.391(0.112,0.613)$

$0.643(0.221,0.862)$

$0.657(0.388,0.823)$ 
Table 11 Continued

Moderate-to-vigorous $\quad 0.543(0.145,0.790) \quad 0.500(0.126,0.750) \quad 0.688(0.334,0.871)$

$0.333(-0.042,0.626)$

$0.518(0.139,0.764) \quad 0.731(0.221,0.927) \quad 0.779(0.469,0.918)$

Sedentary

$0.338(0.090,0.547) \quad 0.467(0.017,0.759)$

$0.764(0.2090 .947)$

$0.206(-0.050,0.437)$

$0.547(0.178,0.781)$

Note. AUC statistics in bold note a significant group difference. 


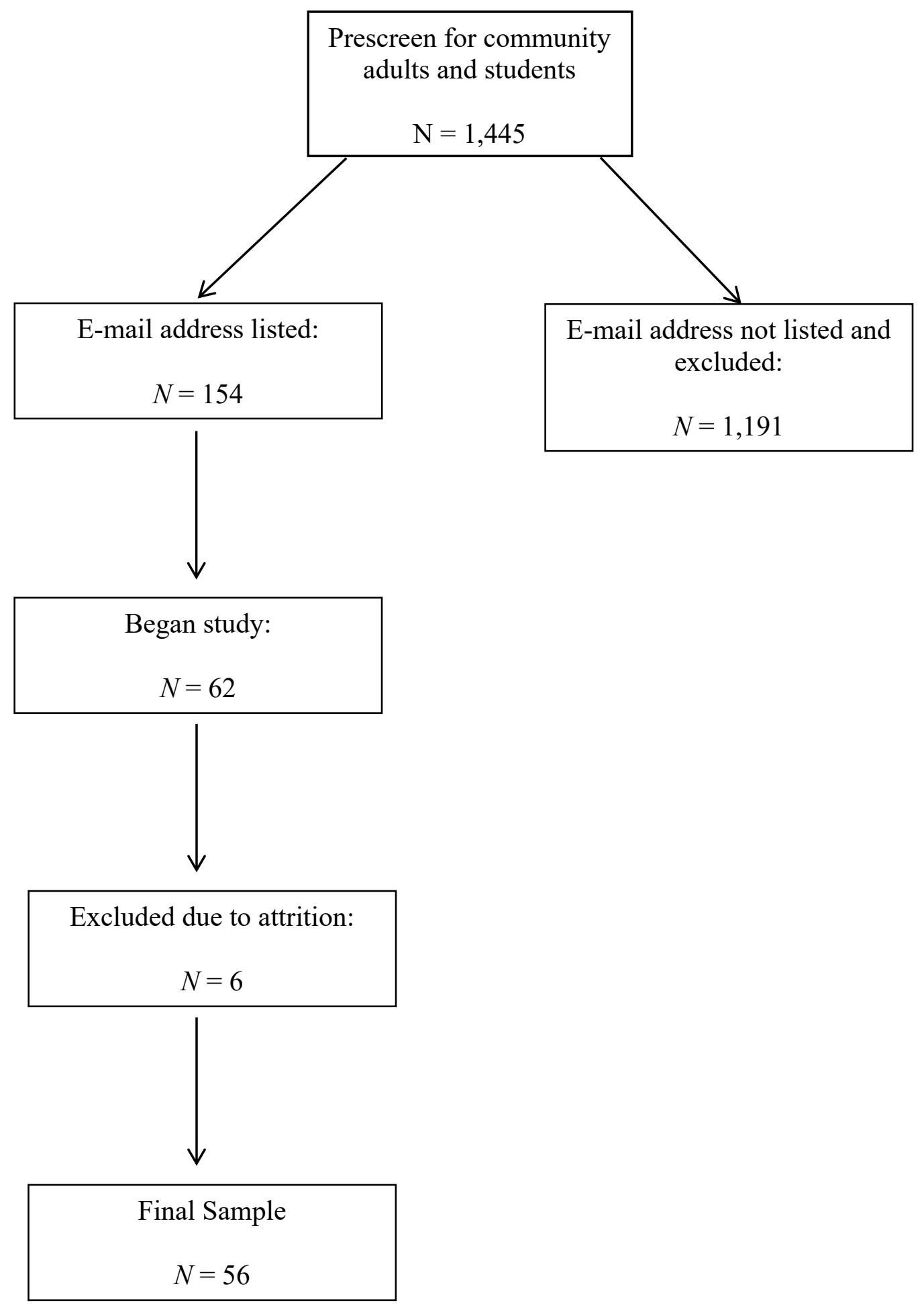

Figure 1. Flowchart detailing the process of inclusion into the study. 


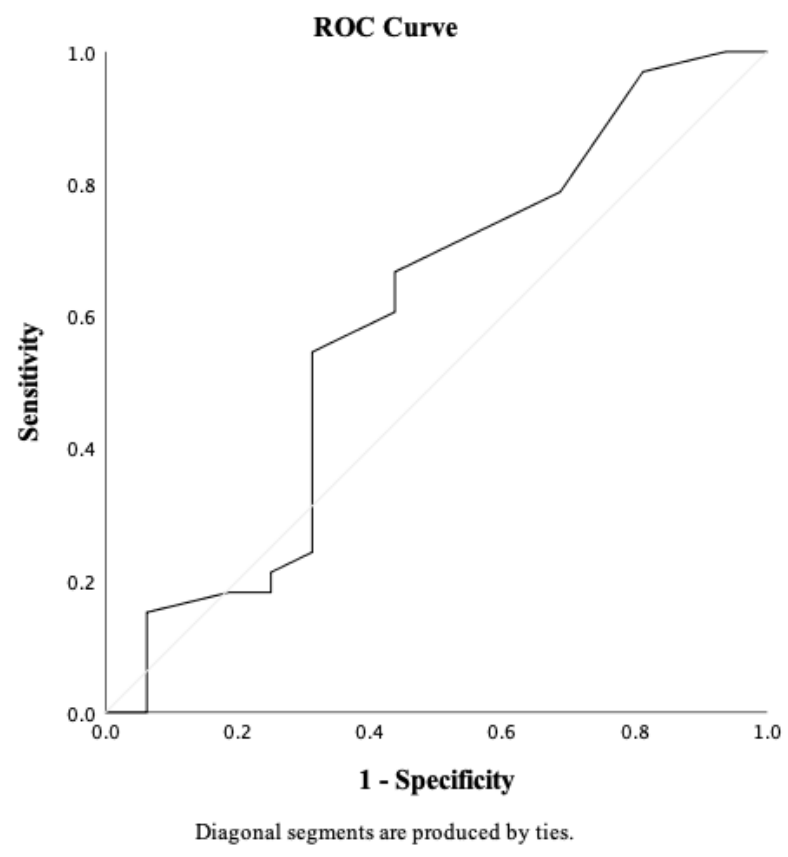

Figure 2. Intensity: ROC curve analysis for Week 2 moderate-to-vigorous-intensity physical activity between the accelerometer and GLTEQ. 


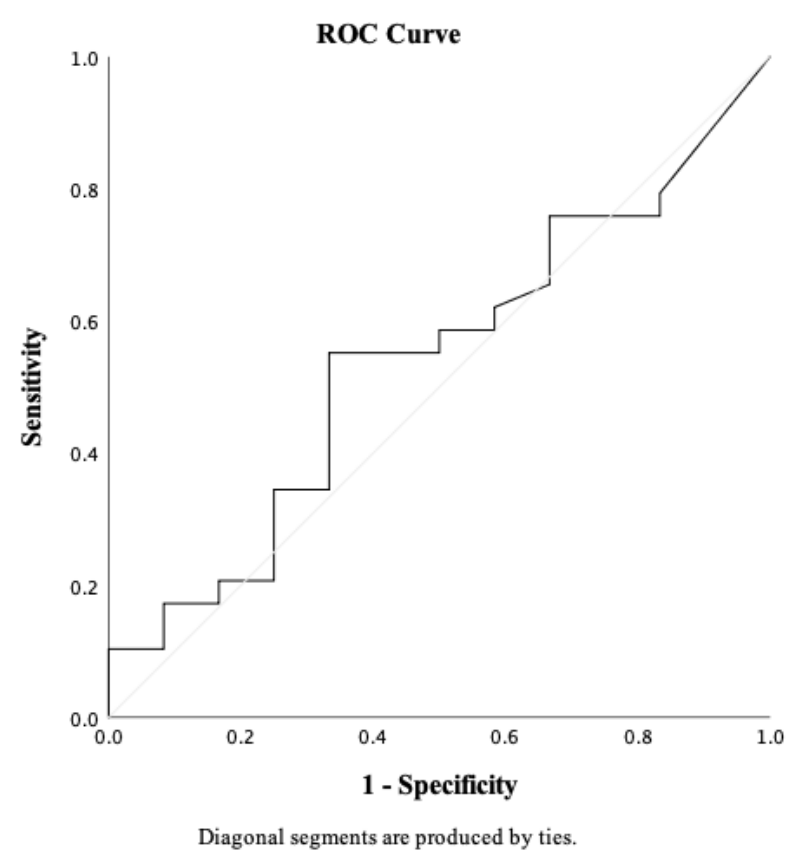

Figure 3. Intensity: ROC curve analysis for Week 2 moderate-to-vigorous-intensity physical activity between the accelerometer and daily diary. 


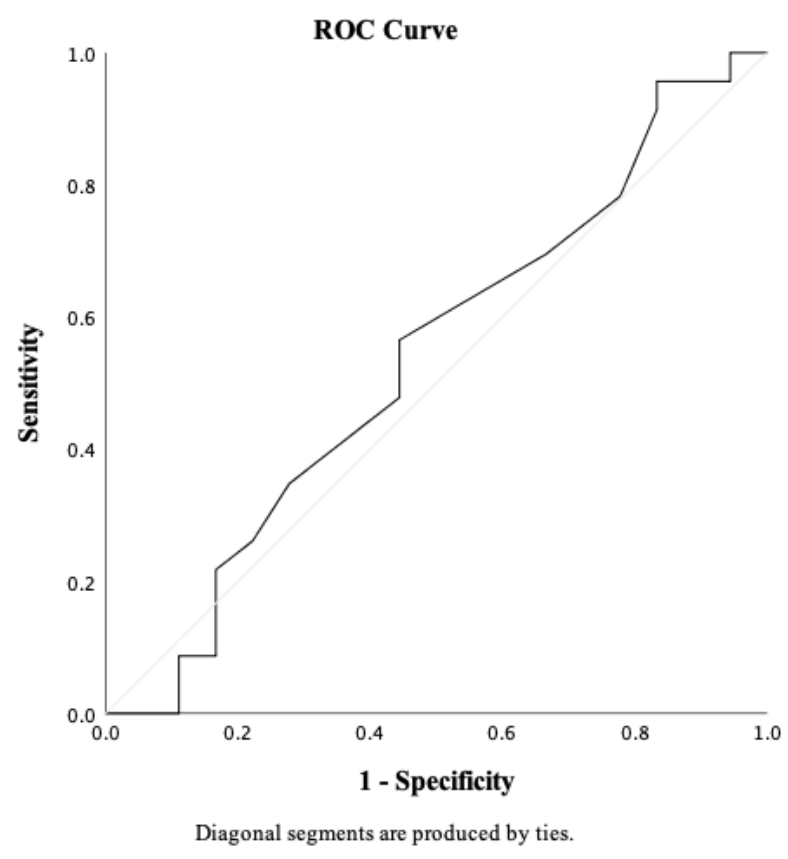

Figure 4. Intensity: ROC curve analysis for Week 2 moderate-to-vigorous-intensity physical activity between the daily diary and GLTEQ. 


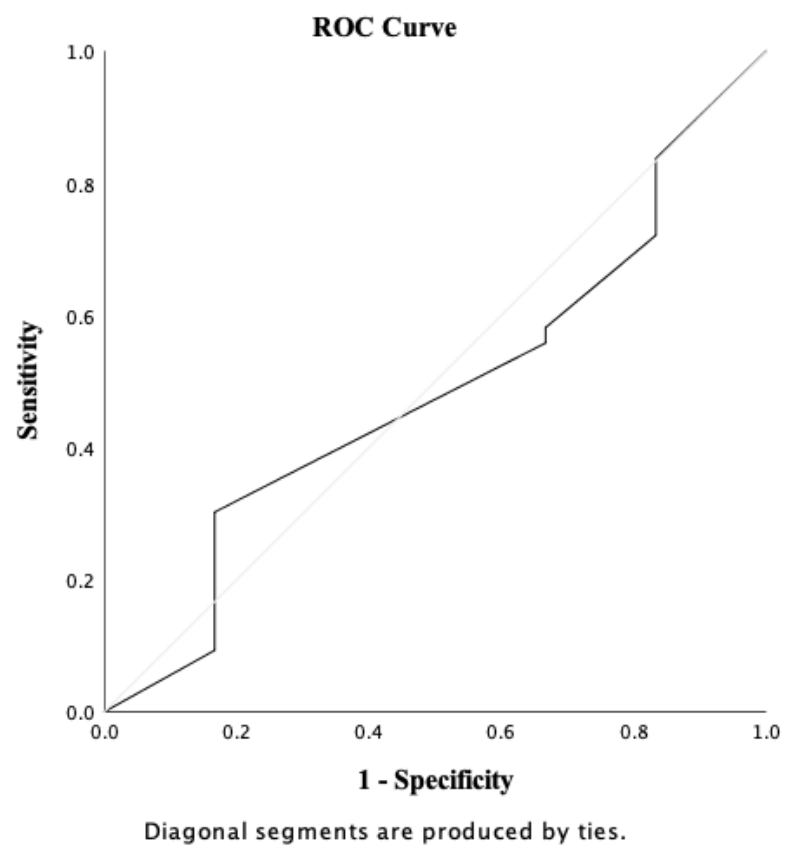

Figure 5. Intensity: ROC curve analysis for Week 2 mild-intensity physical activity between the accelerometer and GLTEQ. 


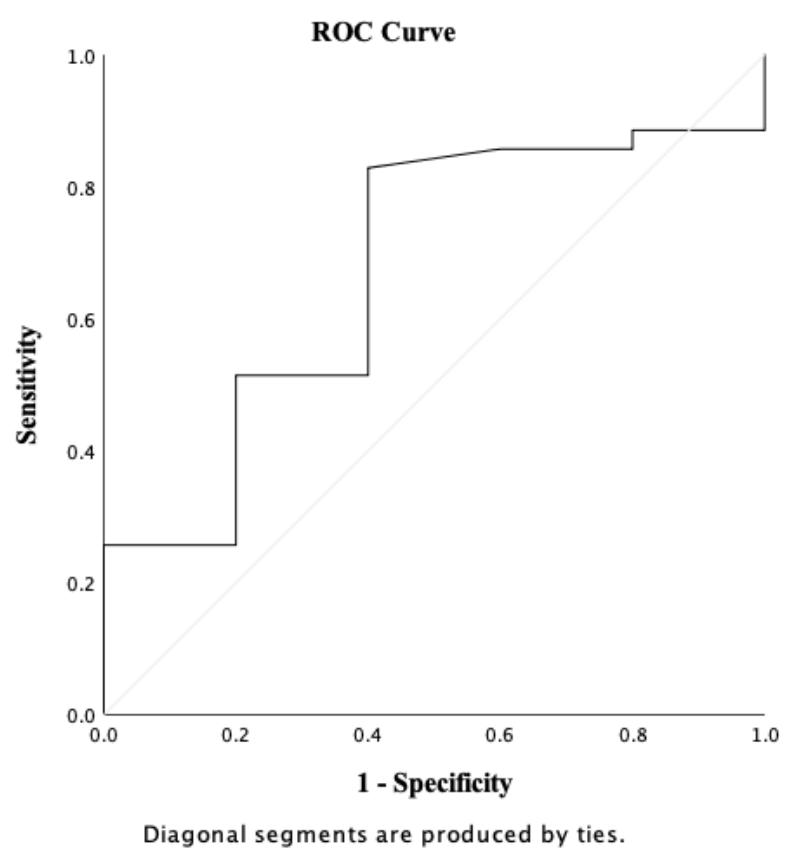

Figure 6. Intensity: ROC curve analysis for Week 2 mild-intensity physical activity between the accelerometer and daily diary. 


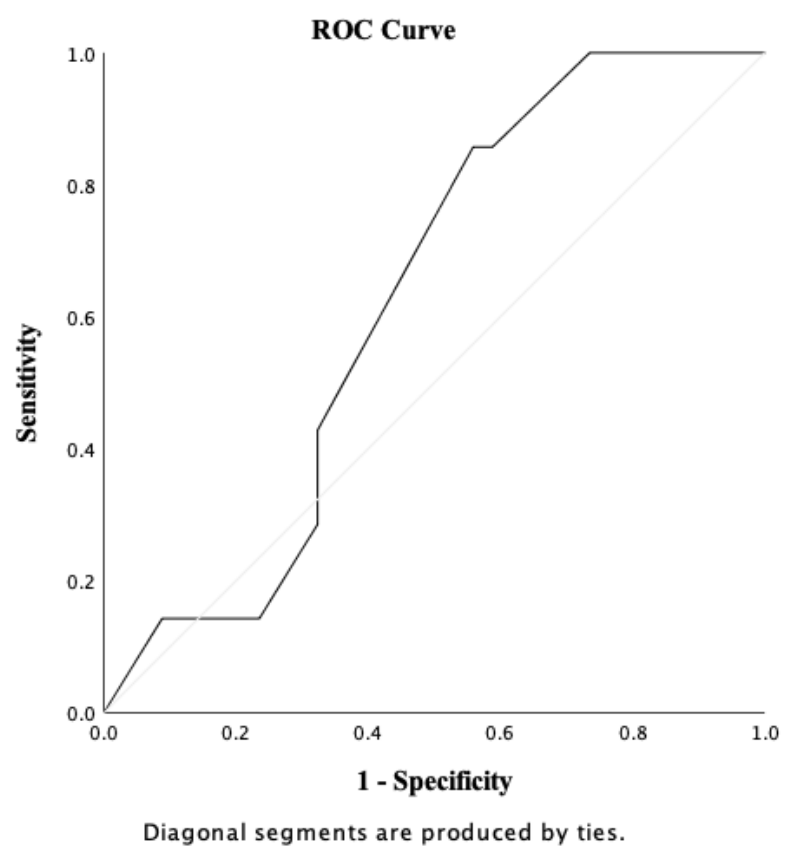

Figure 7. Intensity: ROC curve analysis for Week 2 mild-intensity physical activity between the daily diary and GLTEQ. 


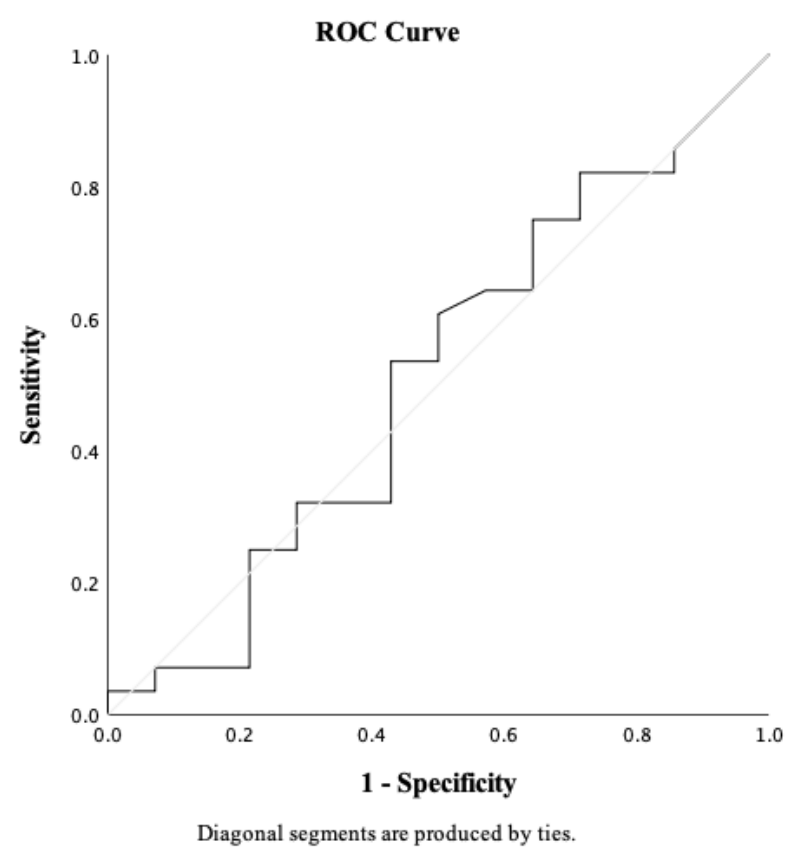

Figure 8. Intensity: ROC curve analysis for Week 2 sedentariness between the accelerometer and daily diary. 


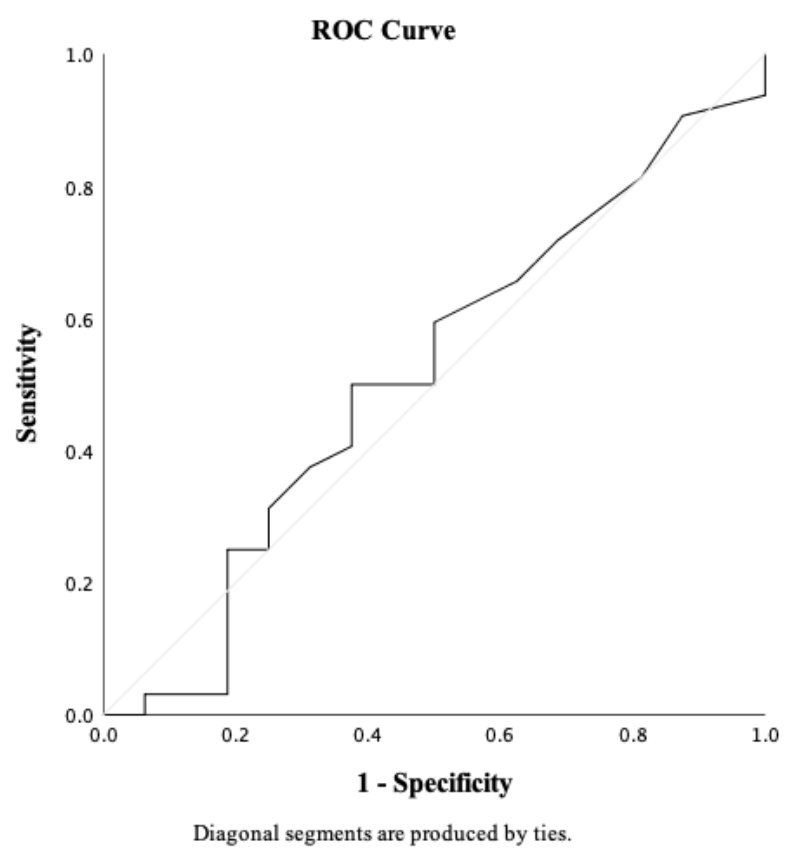

Figure 9. Intensity: ROC curve analysis for Week 3 moderate-to-vigorous-intensity physical activity between the accelerometer and GLTEQ. 


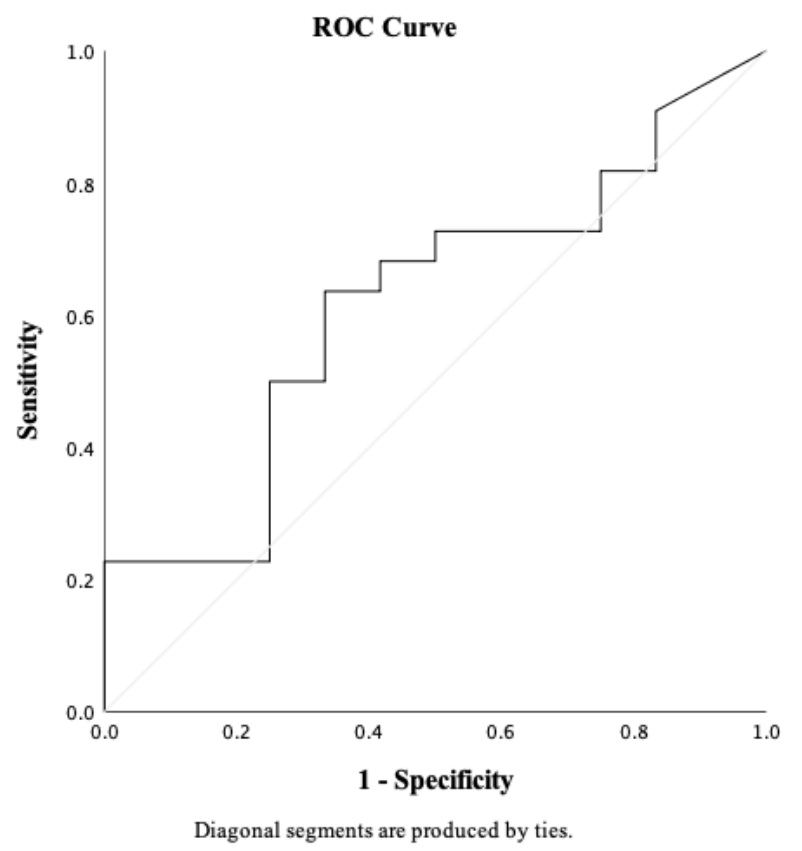

Figure 10. Intensity: ROC curve analysis for Week 3 moderate-to-vigorous-intensity physical activity between the accelerometer and daily diary. 


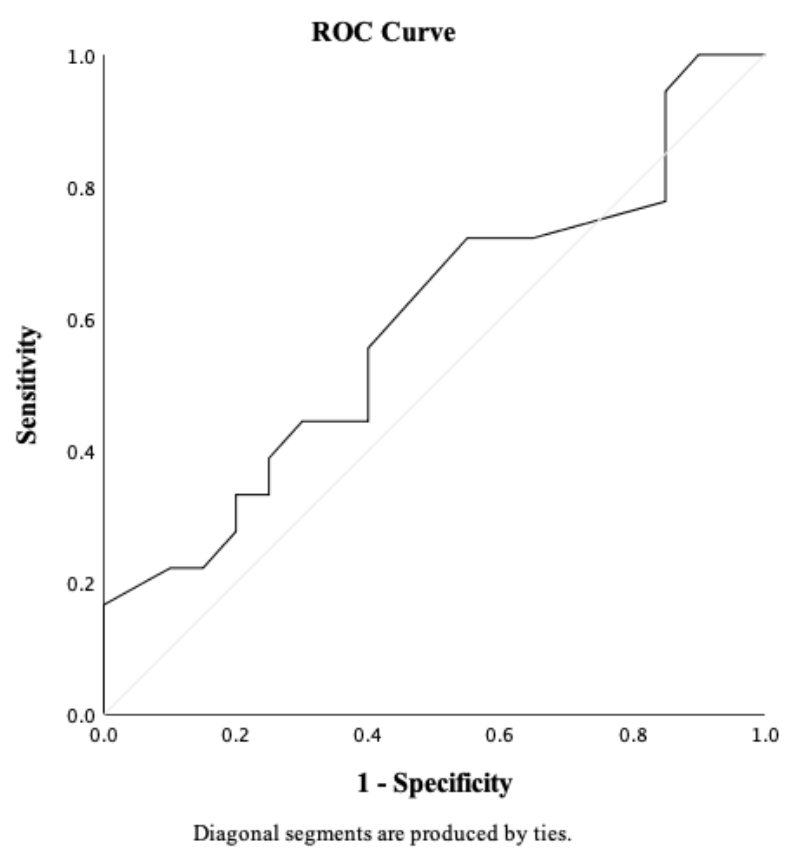

Figure 11. Intensity: ROC curve analysis for Week 3 moderate-to-vigorous-intensity physical activity between the daily diary and GLTEQ. 


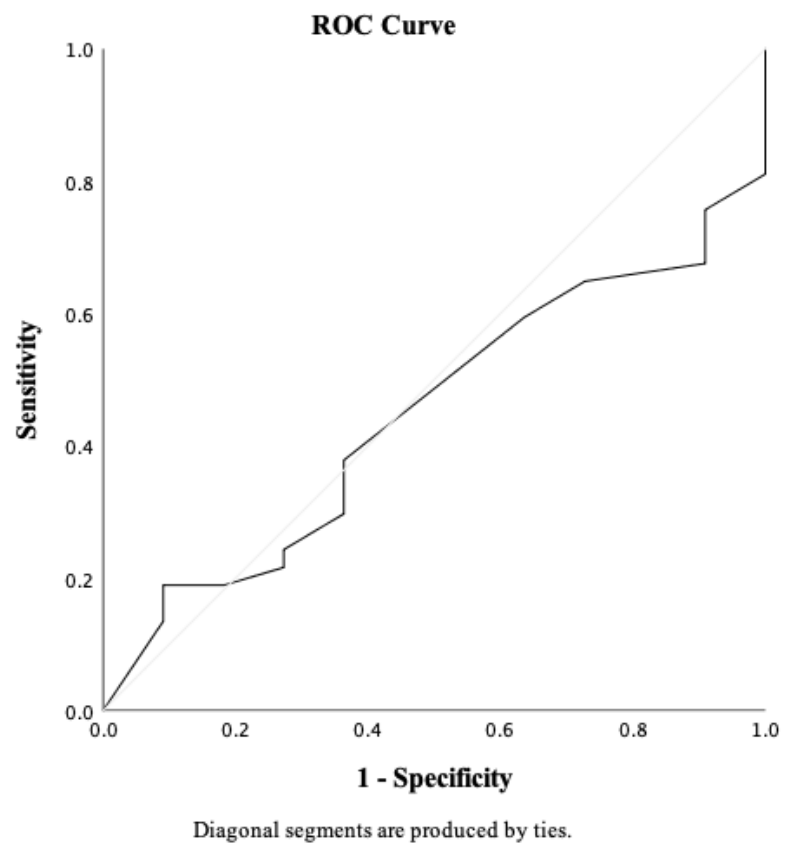

Figure 12. Intensity: ROC curve analysis for Week 3 mild-intensity physical activity between the accelerometer and GLTEQ. 


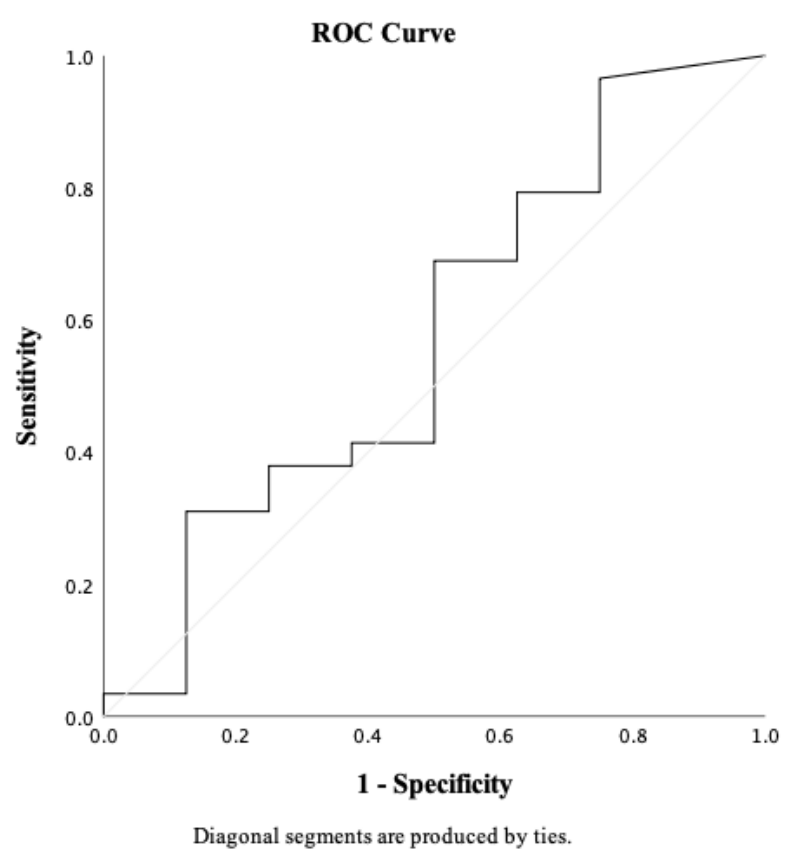

Figure 13. Intensity: ROC curve analysis for Week 3 mild-intensity physical activity between the accelerometer and daily diary. 


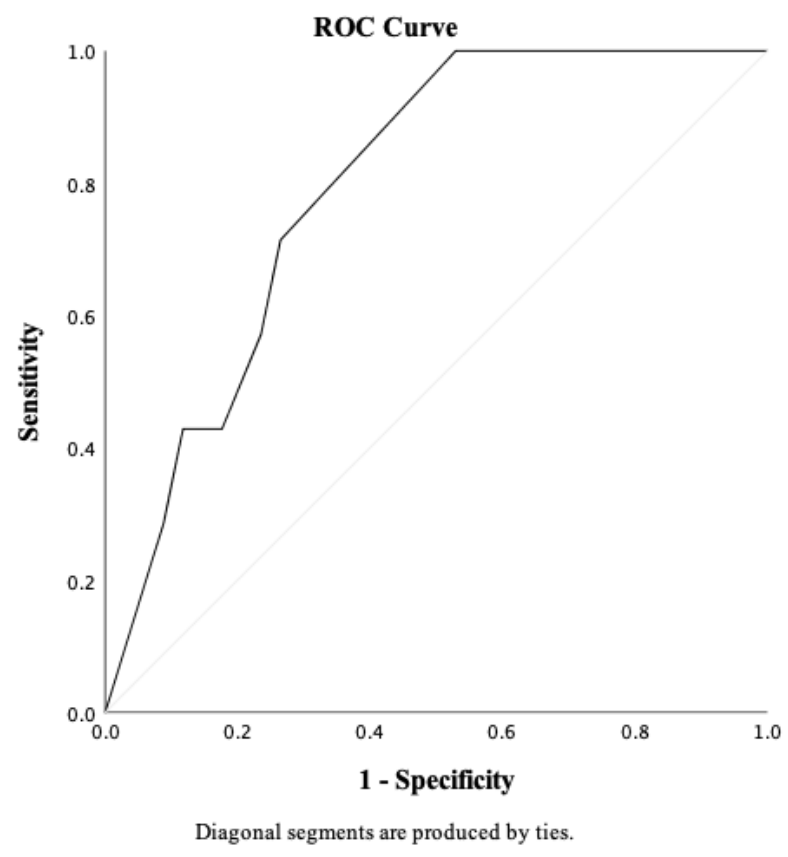

Figure 14. Intensity: ROC curve analysis for Week 3 mild-intensity physical activity between the daily diary and GLTEQ. 


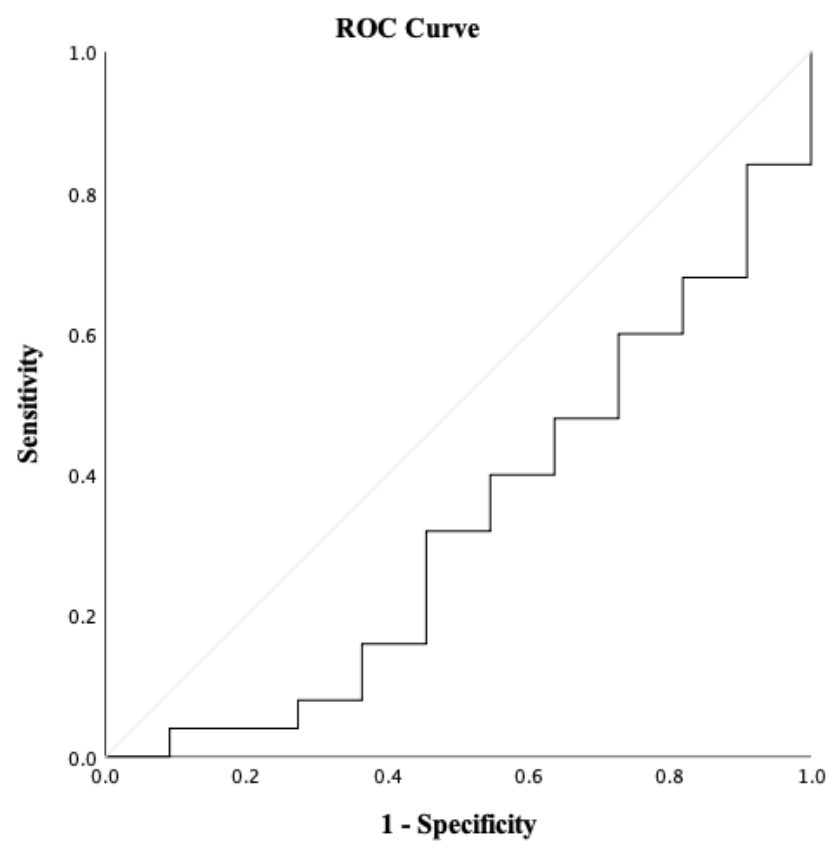

Figure 15. Intensity: ROC curve analysis for Week 3 sedentariness between the accelerometer and daily diary. 


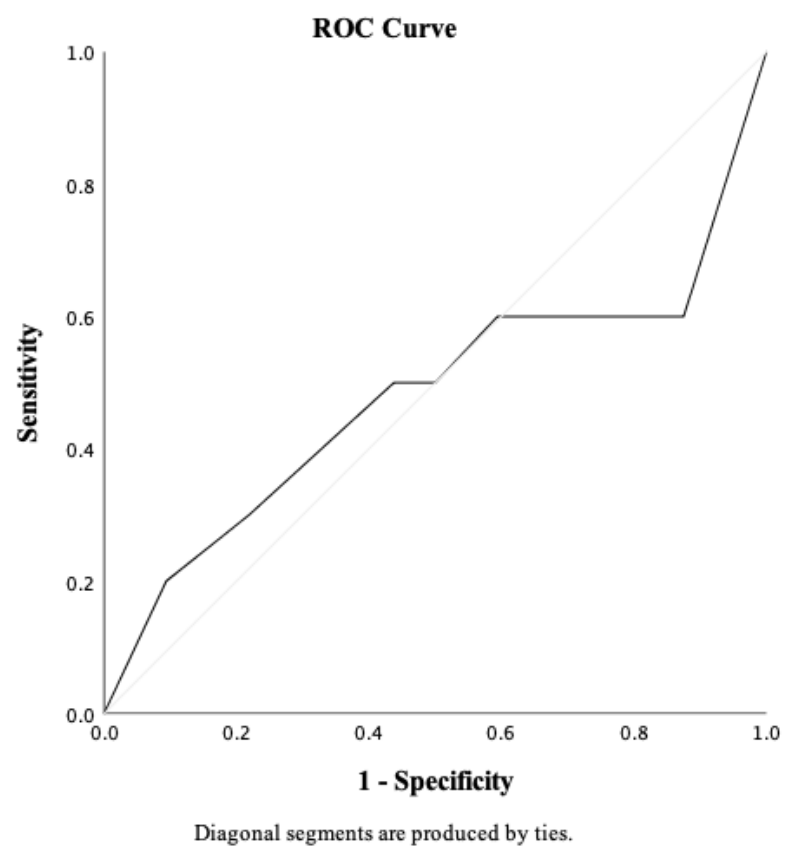

Figure 16. Frequency: ROC curve analysis for Week 2 moderate-to-vigorous-intensity physical activity between the accelerometer and daily diary. 


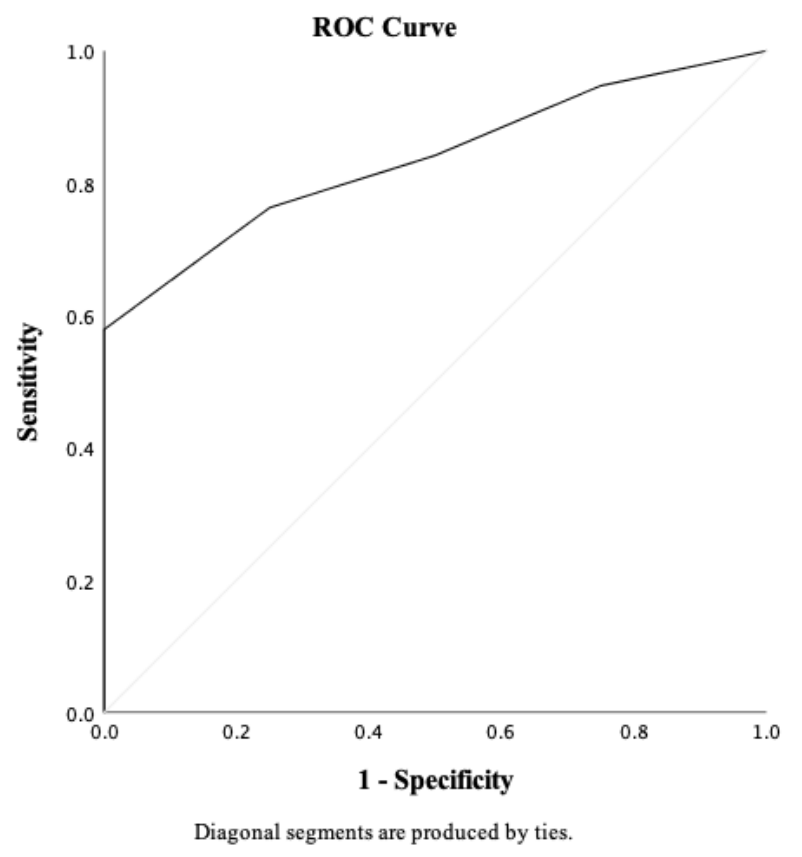

Figure 17. Frequency: ROC curve analysis for Week 2 mild-intensity physical activity between the accelerometer and daily diary. 


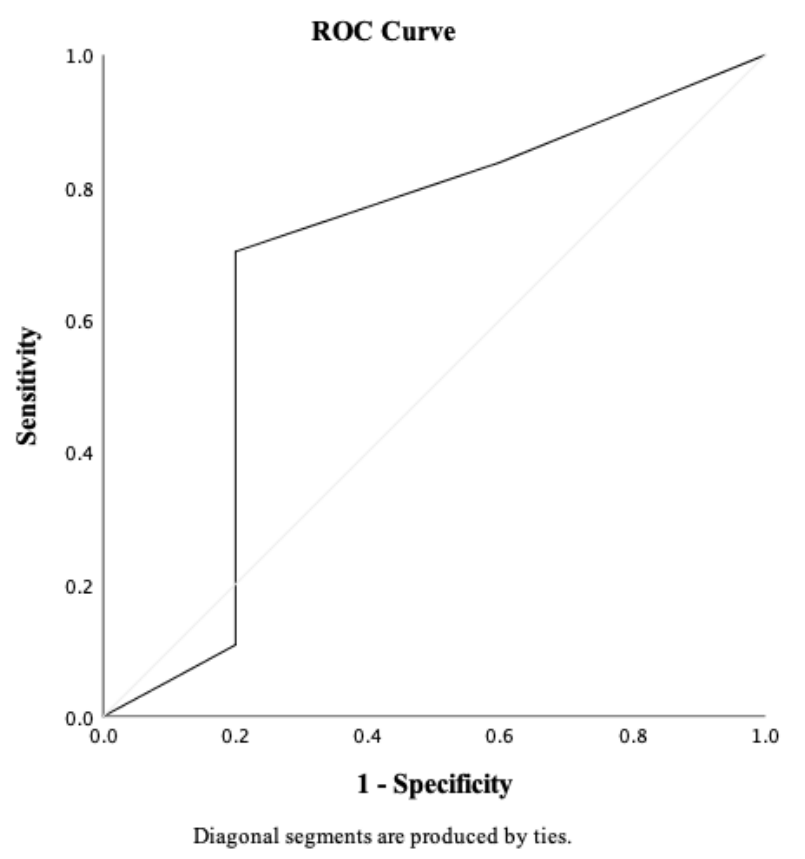

Figure 18. Frequency: ROC curve analysis for Week 2 sedentariness physical activity between the accelerometer and daily diary. 


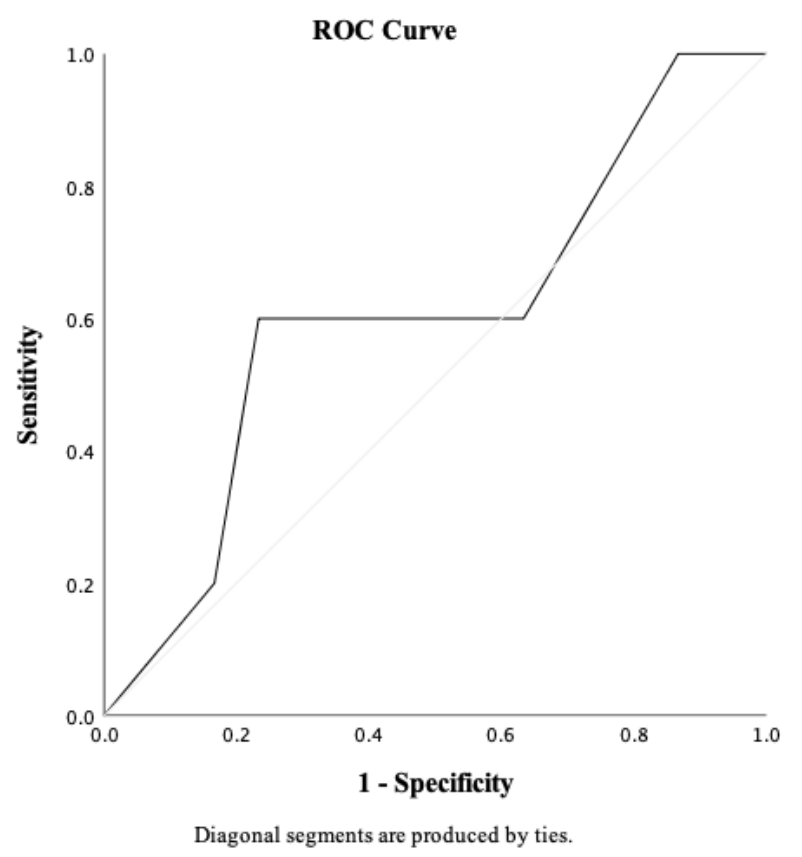

Figure 19. Frequency: ROC curve analysis for Week 3 moderate-to-vigorous-intensity physical activity between the accelerometer and daily diary. 


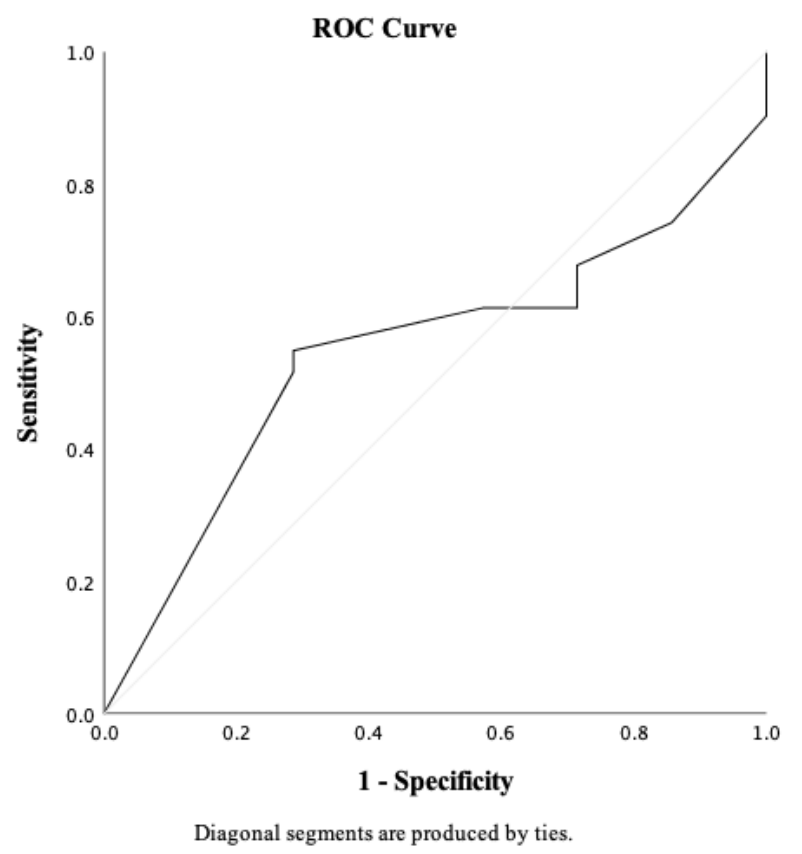

Figure 20. Frequency: ROC curve analysis for Week 3 mild-intensity physical activity between the accelerometer and daily diary. 


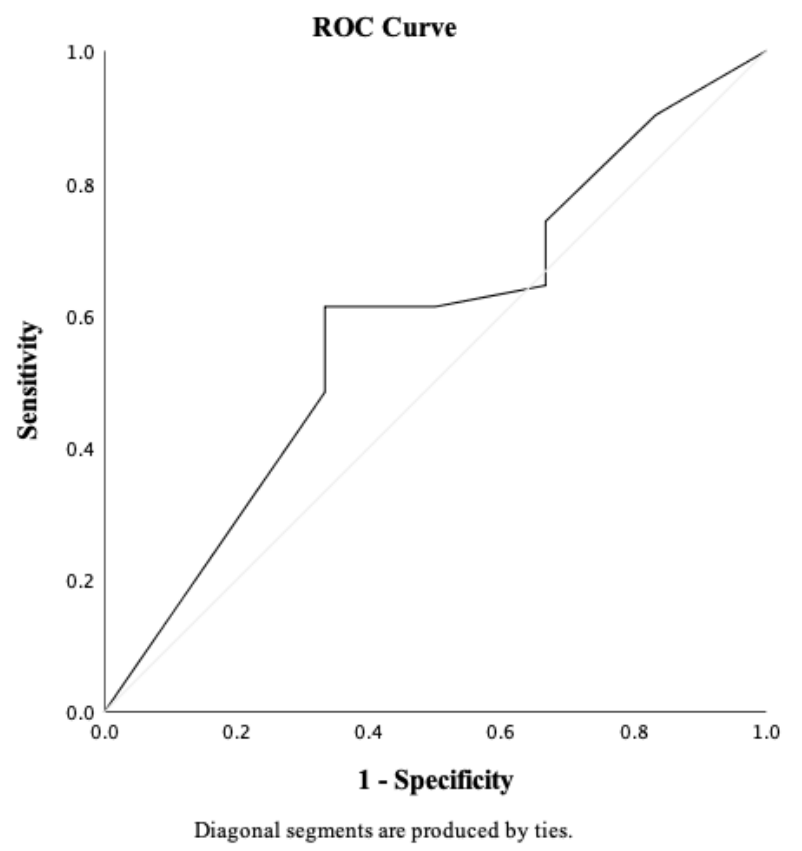

Figure 21. Frequency: ROC curve analysis for Week 3 sedentariness between the accelerometer and daily diary. 


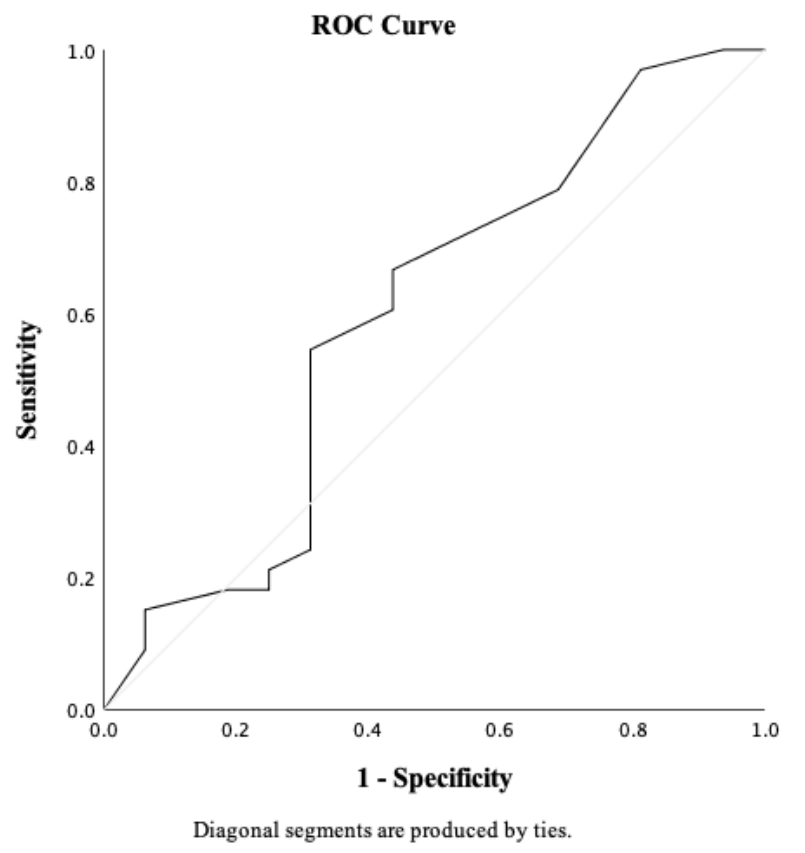

Figure 22. Duration: ROC curve analysis for Week 2 moderate-to-vigorous-intensity physical activity between the accelerometer and GLTEQ. 


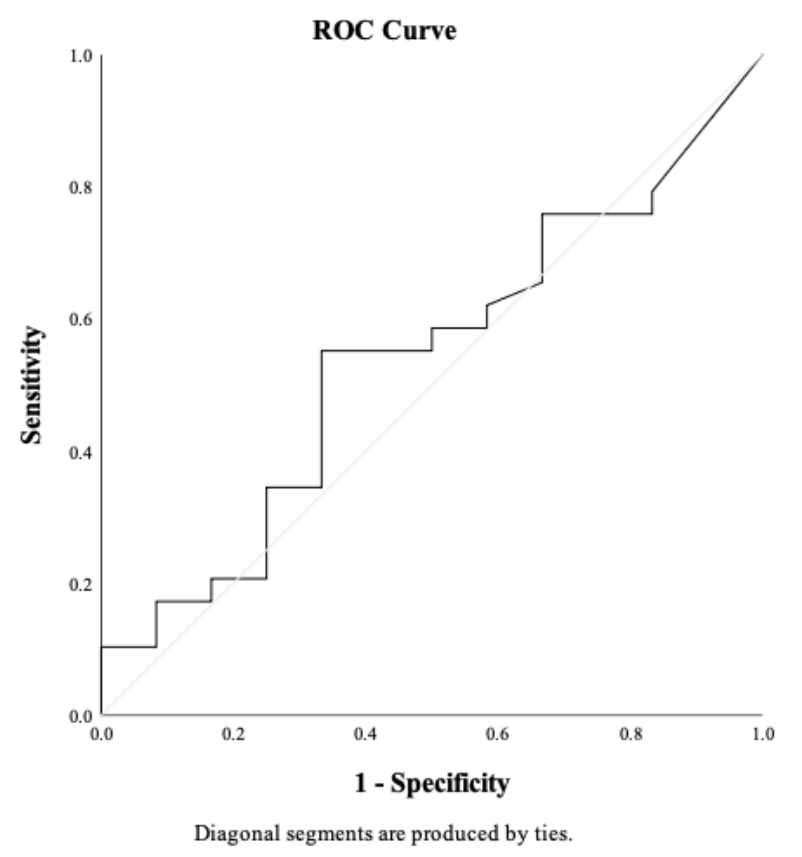

Figure 23. Duration: ROC curve analysis for Week 2 moderate-to-vigorous-intensity physical activity between the accelerometer and daily diary. 


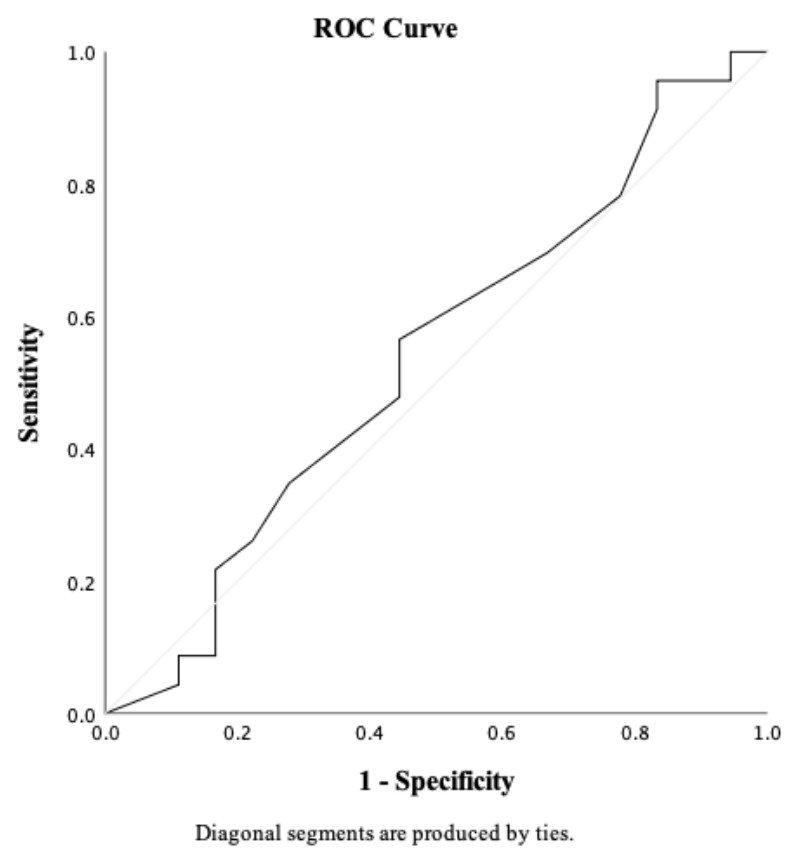

Figure 24. Duration: ROC curve analysis for Week 2 moderate-to-vigorous-intensity physical activity between the daily diary and GLTEQ. 


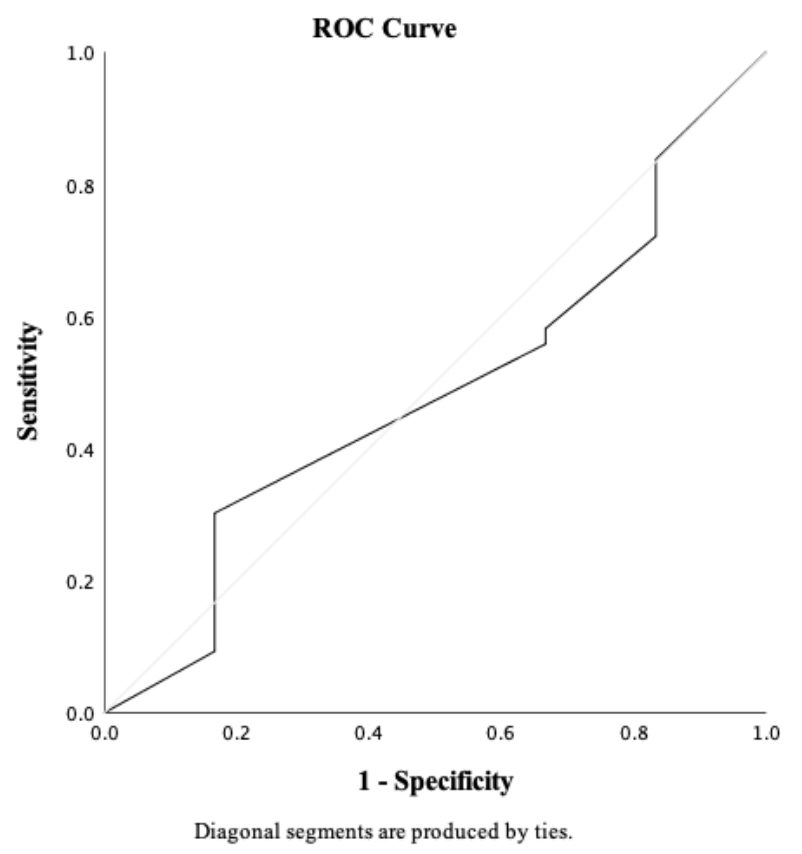

Figure 25. Duration: ROC curve analysis for Week 2 mild-intensity physical activity between the accelerometer and GLTEQ. 


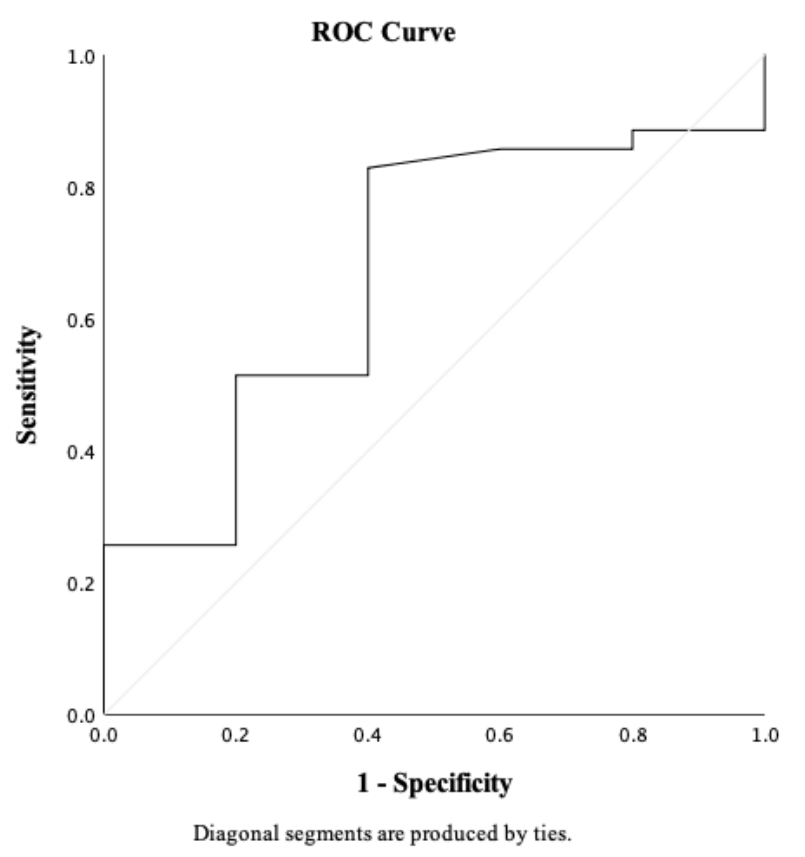

Figure 26. Duration: ROC curve analysis for Week 2 mild-intensity physical activity between the accelerometer and daily diary. 


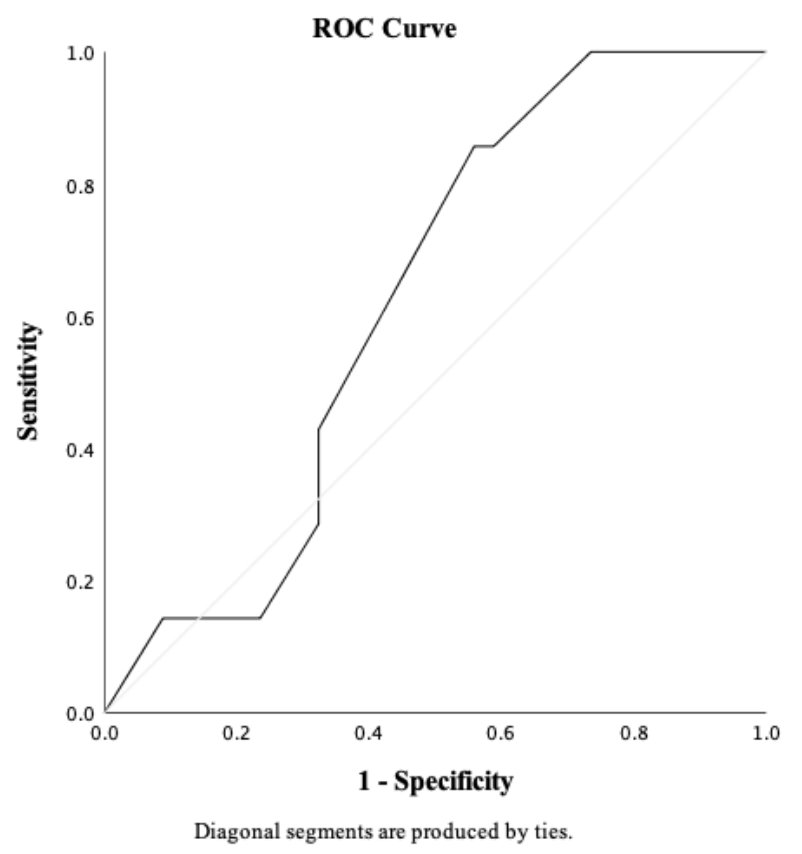

Figure 27. Duration: ROC curve analysis for Week 2 mild-intensity physical activity between the daily diary and GLTEQ. 


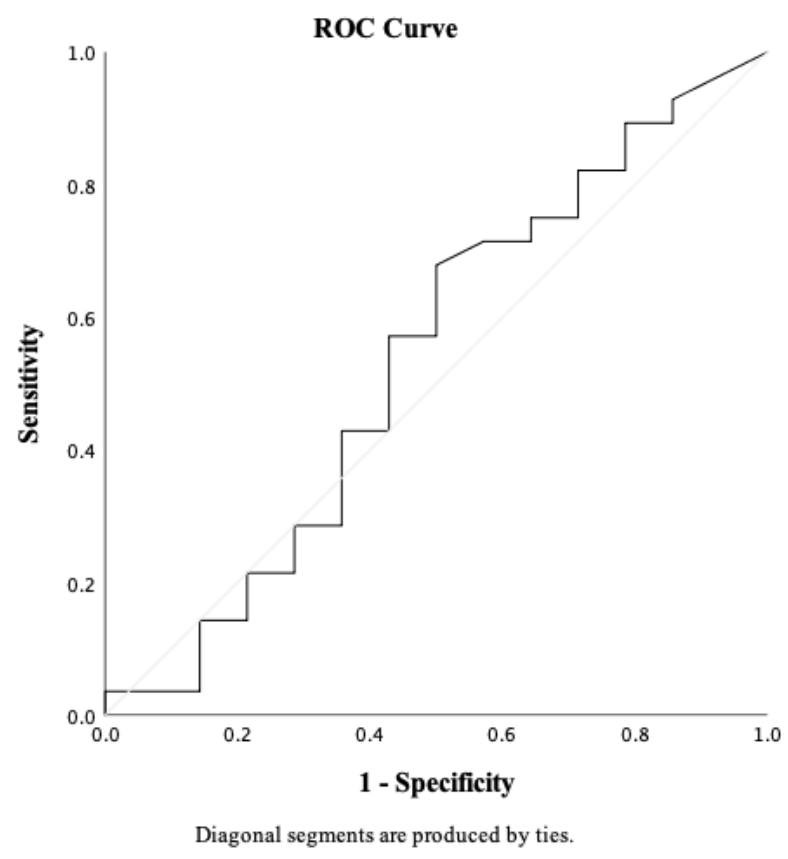

Figure 28. Duration: ROC curve analysis for Week 2 sedentariness between the accelerometer and daily diary. 


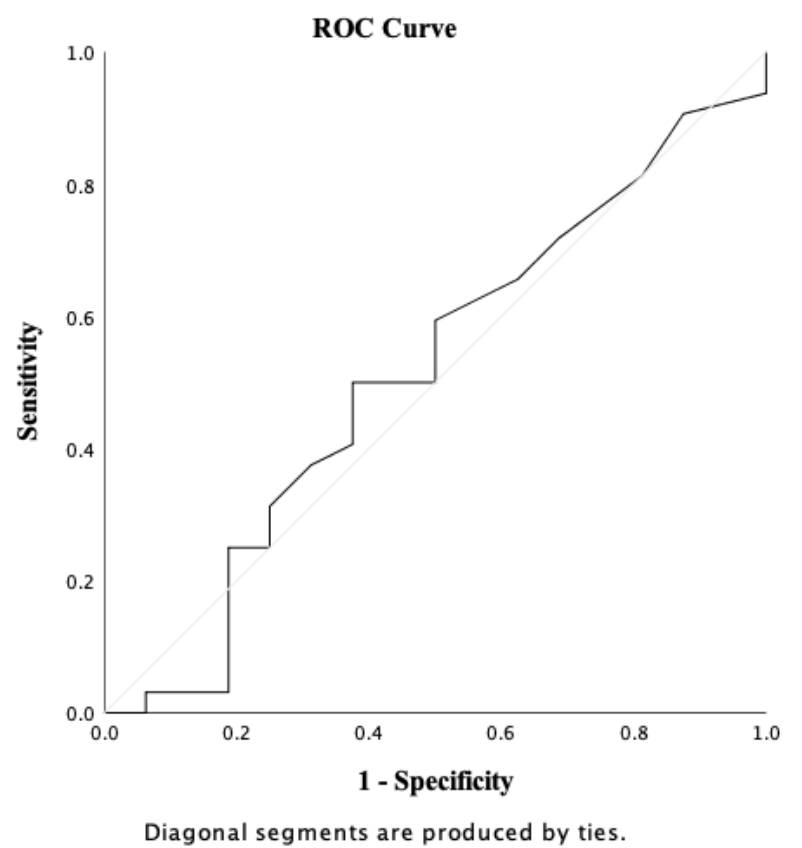

Figure 29. Duration: ROC curve analysis for Week 3 moderate-to-vigorous-intensity physical activity between the accelerometer and GLTEQ. 


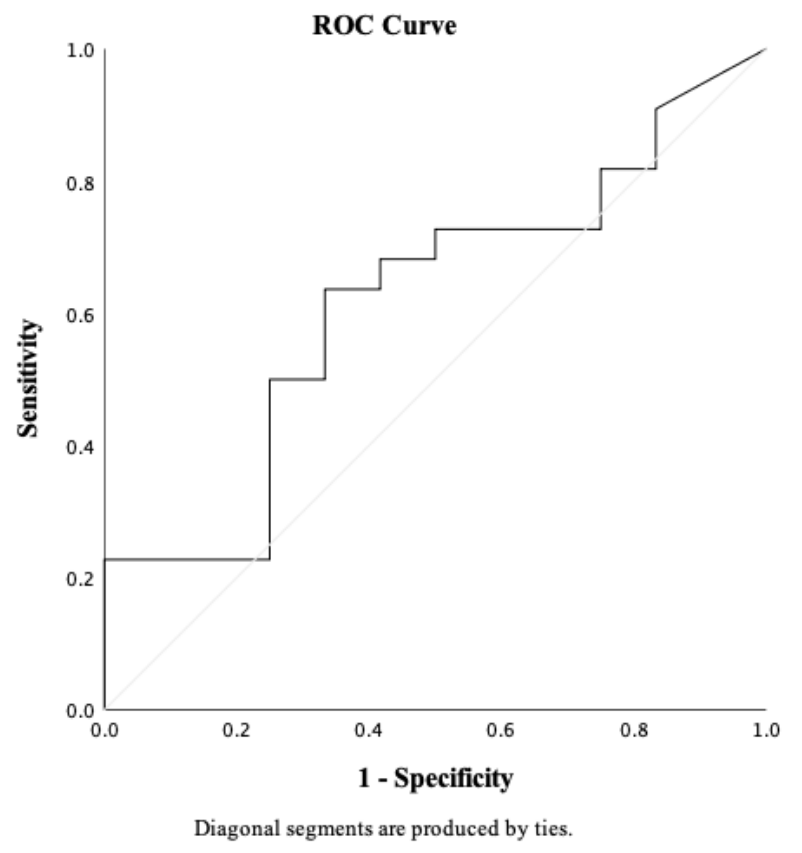

Figure 30. Duration: ROC curve analysis for Week 3 moderate-to-vigorous-intensity physical activity between the accelerometer and daily diary. 


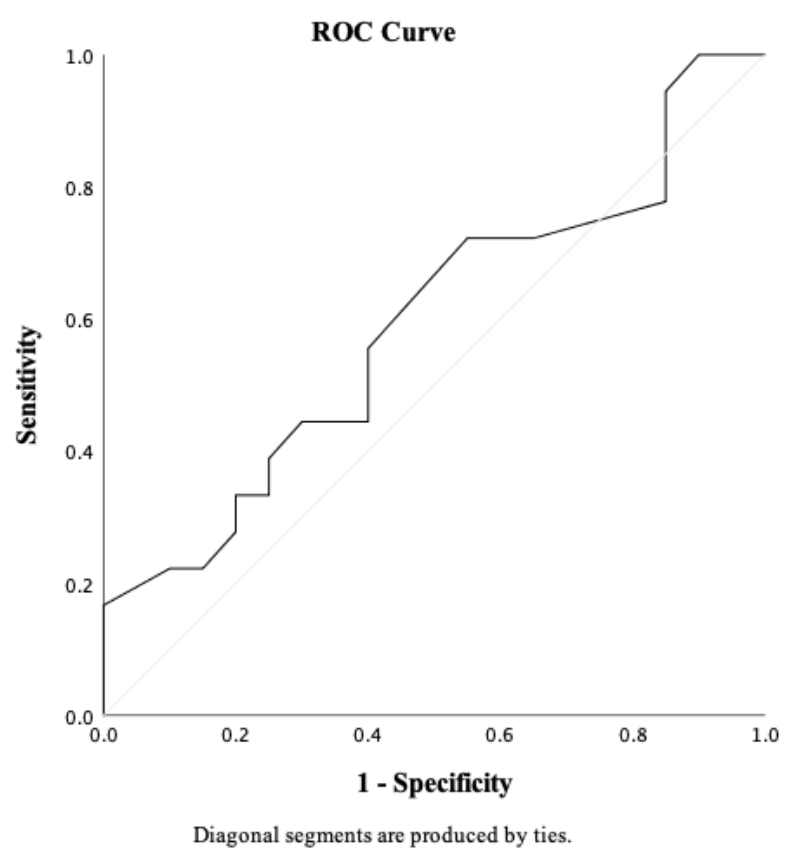

Figure 31. Duration: ROC curve analysis for Week 3 moderate-to-vigorous-intensity physical activity between the daily diary and GLTEQ. 


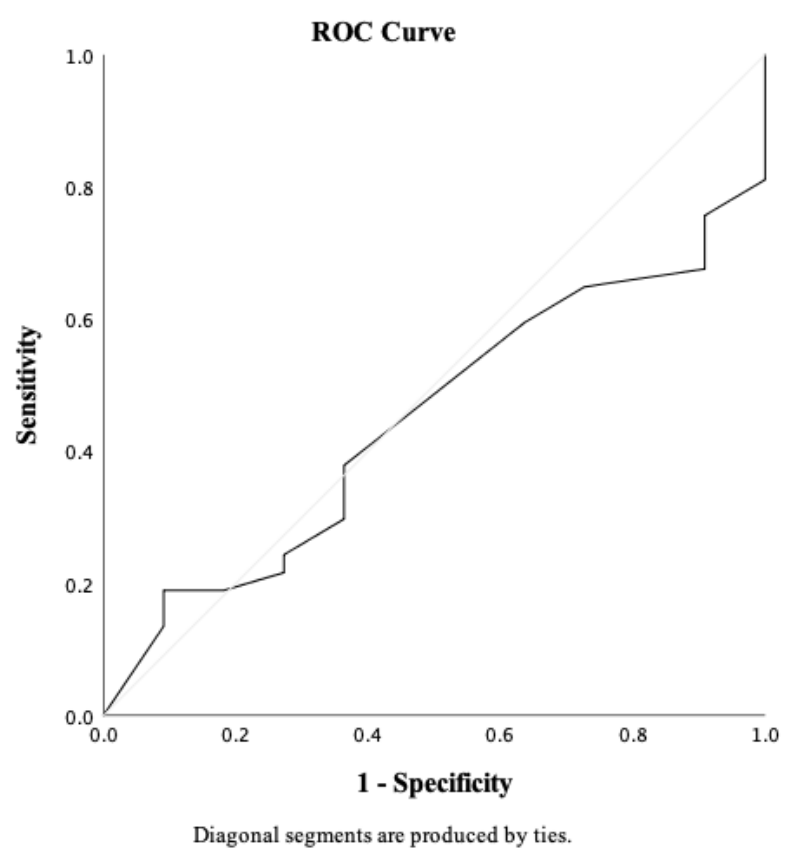

Figure 32. Duration: ROC curve analysis for Week 3 mild-intensity physical activity between the accelerometer and GLTEQ. 


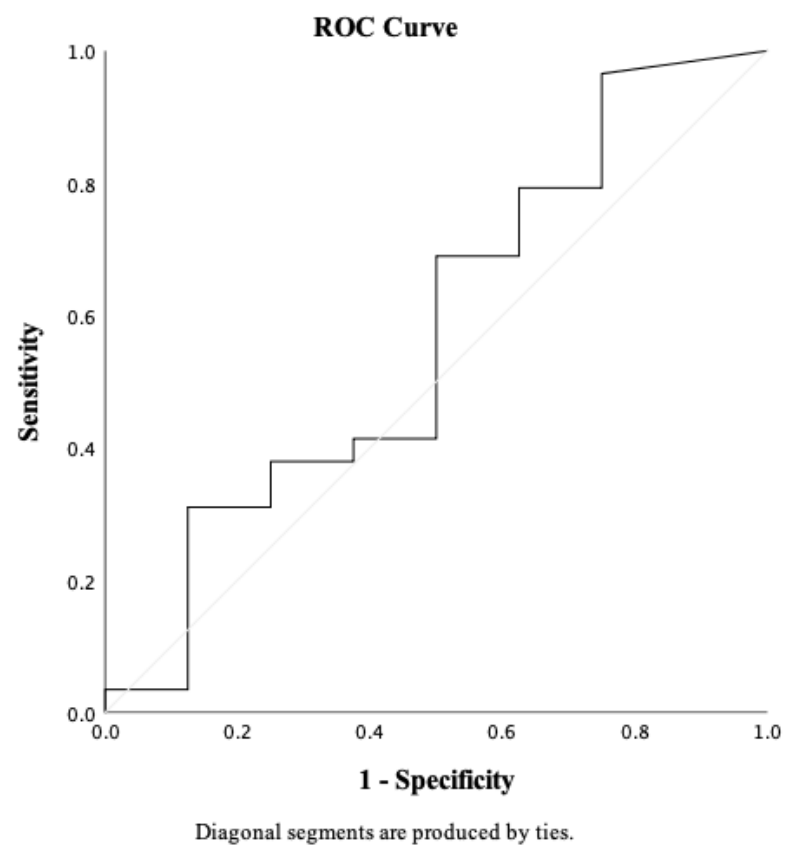

Figure 33. Duration: ROC curve analysis for Week 3 mild-intensity physical activity between the accelerometer and daily diary. 


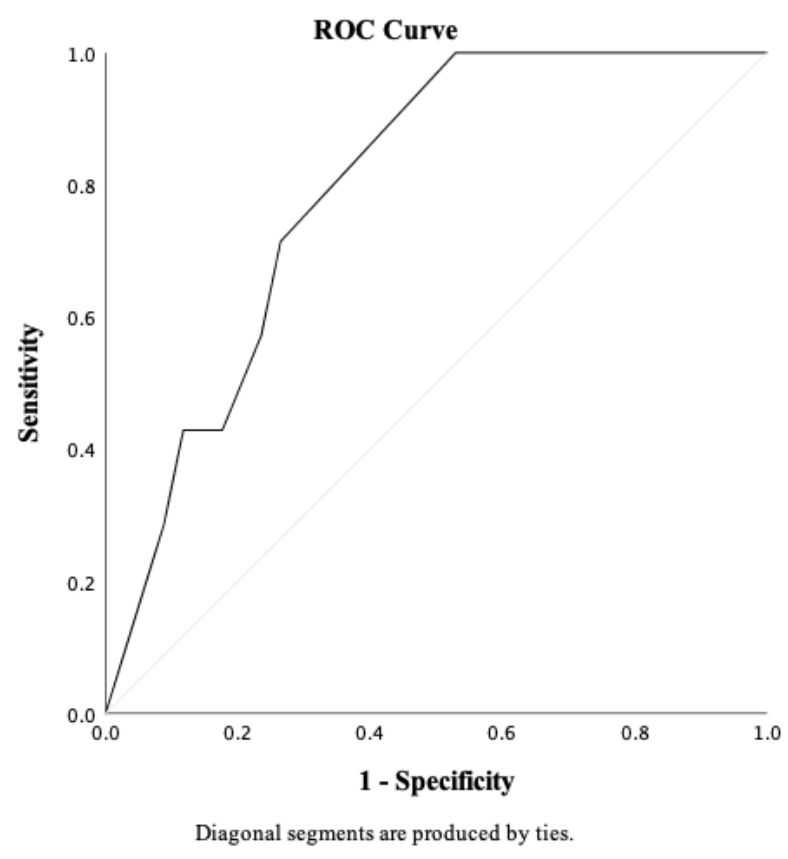

Figure 34. Duration: ROC curve analysis for Week 3 mild-intensity physical activity between the daily diary and GLTEQ. 


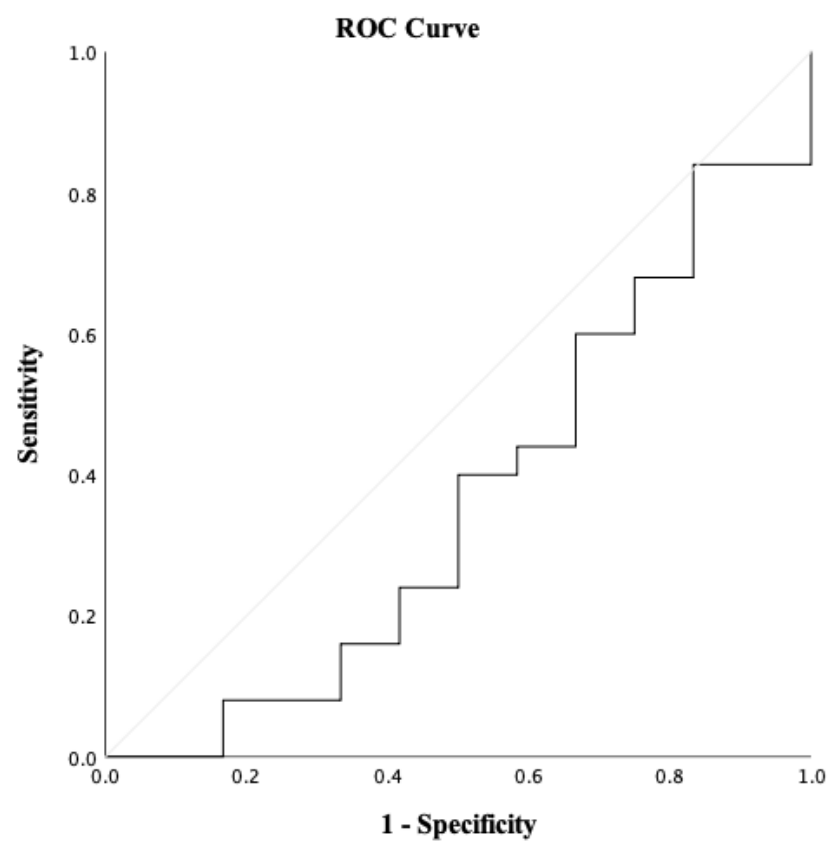

Figure 35. Duration: ROC curve analysis for Week 3 sedentariness between the accelerometer and daily diary. 


\section{Appendix A}

\section{ABCD Prescreen and Introduction to Study}

Part 1: Participants first completed an online prescreen to be considered for inclusion in the study. The prescreen contained measures of various domains, including emotion, cognition, social relationships, and physical activity. Prompts in the prescreen directly asked potential participants if they had short-term goals that they wished to work on, if they would be interested in working with a health coach on these goals, and to leave their e-mail address if they wished to be contacted about participating in the three-week protocol.

Part 2: If participants answered with the intent of wishing to work with a health coach on their short-term goals and they left their e-mail address, the PI of the study later e-mailed them, introducing them to their health coach.

Part 3: The health coach assigned to each participant then scheduled the first meeting, starting the three-week protocol, which was when participants received their MyBasis accelerometer. Note: If respondents noted on the prescreen that they were interested in participating in the full study, but did not leave an e-mail address, they were not contacted for the next portion of the study. 


\section{Appendix B}

The Goal-Striving Reasons Index (Ehrlich, 2012)

A “*” indicates the item was part of the sub-scale utilized to assess intrinsic motivation.

$$
\begin{aligned}
& 1 \text { - Not at all true } \\
& 2 \text { - Untrue } \\
& 3 \text { - Slightly untrue } \\
& 4 \text { - Neither agree nor disagree } \\
& 5 \text { - Slightly true } \\
& 6 \text { - True } \\
& 7 \text { - Very true }
\end{aligned}
$$

How true are each of the follow reasons (in terms of your physical activity goals)? Using this responses card, would you say that:

1. this goal is important to you because if you fail, your reputation among other people would drop.

2. this goal is important to you because you're having fun working on this goal.*

3. this goal is important to you because if you fail, your self-esteem would really suffer.

4. this goal is important to you because it helps others.

5. this goal is important to you because of the money.

6. this goal is important to you because you enjoy doing it.*

7. this goal is important to you because if you fail, other people would look down on you.

8. this goal is important to you because it serves a good cause.

9. this goal is important to you because it helps you to earn a living

10. this goal is important to you because if you fail, you would feel like a loser.

11. this goal is important to you because you actually enjoy working on this goal quite a lot.*

12. this goal is important to you because other people do benefit from it.

13. this goal is important to you because it helps you to make a living. 
14. this goal is important to you because if you fail, you could not look yourself in the eyes.

15. this goal is important to you because you get a lot of energy from the goal.*

16. this goal is important to you because it makes ends meet. 


\section{Appendix C}

\section{My Basis Accelerometers}

Participants wore the MyBasis accelerometer over the 21-day protocol. It recorded their daily steps, heart rate, calories burned, sleep, intensity, duration, and frequency of physical activity.

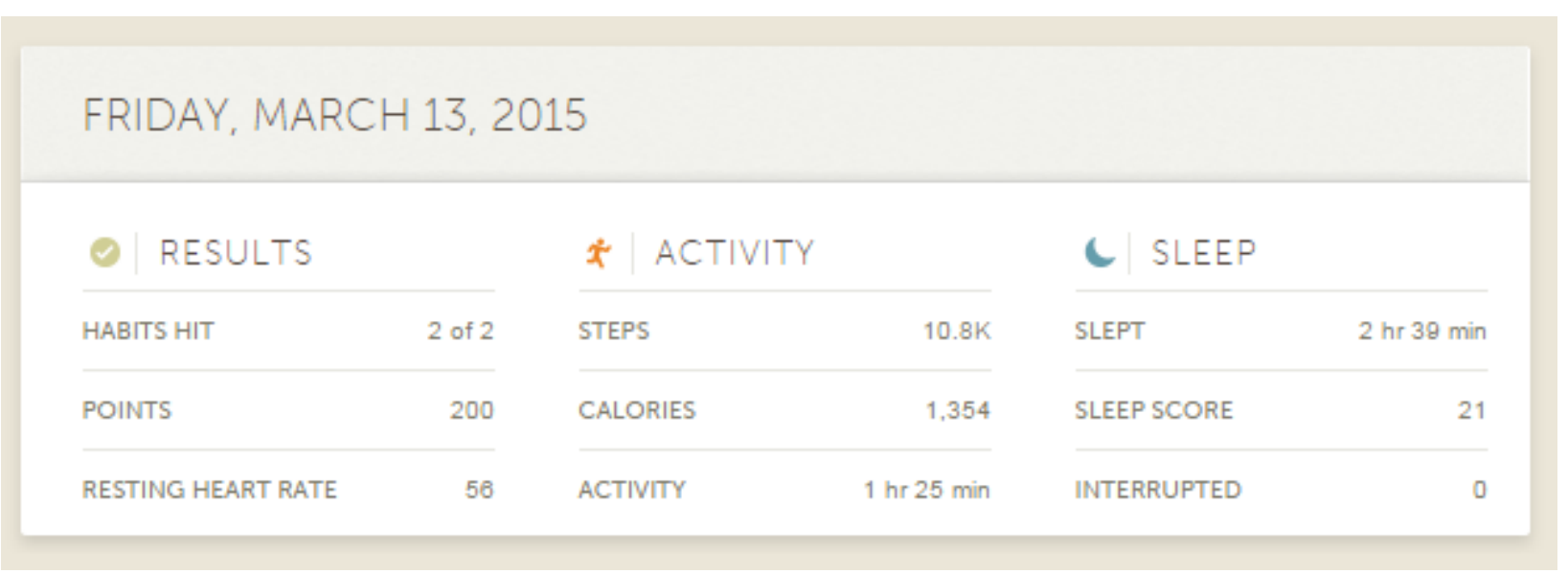




\section{Appendix D}

The Godin Leisure Time Exercise Questionnaire (Godin \& Shephard, 1997)

During a typical 7-Day period (a week), how many times on the average do you do the following kinds of exercise for more than 15 minutes during your free time:

1. Strenuous Exercise (heart beats rapidly) (e.g., running, jogging, hockey, football, soccer, squash, basketball, cross country skiing, judo, roller skating, vigorous swimming, vigorous long-distance bicycling)

2. Moderate Exercise (not exhausting) (e.g., fast walking, baseball, tennis, easy bicycling, volleyball, badminton, easy swimming, alpine skiing, popular and folk dancing)

3. Mild Exercise (minimal effort) (e.g., yoga, archery, fishing from river bank, bowling, horseshoes, golf, snow-mobiling, easy walking) 


\section{Appendix E}

\section{Daily Diary Assessment}

As is denoted by the "Time" header, participants received one of these two questions four times a day to assess physical activity intensity, duration, and frequency.

Time 1

Q7 Since you woke up today, how many minutes of each activity did you engage in?

Vigorous Activity (enough to sweat) :

Moderate Activity (not exhausting) :

Light Activity (minimal effort) :

Sedentary Activity (sitting still) :

Total :

Times 2, 3, \& 4

Q 7 Since the last session, how many minutes of each activity did you engage in?

Vigorous Activity (enough to sweat) :

Moderate Activity (not exhausting) :

Light Activity (minimal effort) :

Sedentary Activity (sitting still) :

Total : 
Appendix F

Research Questions and Hypotheses

\begin{tabular}{|c|c|c|}
\hline $\begin{array}{l}\text { Research } \\
\text { Question 1 }\end{array}$ & $\begin{array}{l}\text { Are the subjective measures (GLTEQ and diary } \\
\text { report) associated with the objective measure } \\
\text { (accelerometer)? }\end{array}$ & \\
\hline Hypothesis 1a & $\begin{array}{l}\text { It was hypothesized that reports from the GLTEQ } \\
\text { and daily diaries would over-report physical activity } \\
\text { intensity (moderate-to-vigorous, mild, and } \\
\text { sedentary), duration, and frequency in comparison } \\
\text { to data gathered from the accelerometer. }\end{array}$ & $\begin{array}{l}\text { Largely not } \\
\text { supported }\end{array}$ \\
\hline Hypothesis $1 \mathrm{~b}$ & $\begin{array}{l}\text { It was hypothesized that the daily diary method, in } \\
\text { comparison to the GLTEQ, would exhibit the } \\
\text { strongest agreement with the accelerometer data, in } \\
\text { regards to labeling physical activity intensity } \\
\text { (moderate-to-vigorous and mild) and duration. }\end{array}$ & Partially supported \\
\hline Hypothesis 1c & $\begin{array}{l}\text { It was hypothesized that the relation between the } \\
\text { GLTEQ and the accelerometer would be weak }(\mathrm{r}< \\
\text {.30) for mild intensities and duration. The relation } \\
\text { between the GLETQ and accelerometer would be } \\
\text { weak-to-medium }(.10<\mathrm{r}<.49) \text { for moderate-to- } \\
\text { vigorous physical activity intensity and duration. }\end{array}$ & $\begin{array}{l}\text { Largely not } \\
\text { supported }\end{array}$ \\
\hline Hypothesis 1d & $\begin{array}{l}\text { It was hypothesized that the relation between the } \\
\text { daily diary reports and the accelerometer would be } \\
\text { weak-to-medium }(.10<\mathrm{r}<.49) \text { for intensity } \\
\text { (moderate-to-vigorous, mild, and sedentary), } \\
\text { duration, and frequency. }\end{array}$ & Partially supported \\
\hline Hypothesis 1e & $\begin{array}{l}\text { The association between the GLTEQ and daily } \\
\text { diary report was hypothesized to be medium-to- } \\
\text { strong }(.29<\mathrm{r}<1.00) \text { for intensity (moderate-to- } \\
\text { vigorous and mild) and duration. }\end{array}$ & Partially supported \\
\hline $\begin{array}{l}\text { Research } \\
\text { Question 2 }\end{array}$ & $\begin{array}{l}\text { Does age influence agreement for intensity, } \\
\text { duration, and frequency across the accelerometer, } \\
\text { daily diary, and GLTEQ? }\end{array}$ & \\
\hline Hypothesis $2 \mathrm{a}$ & $\begin{array}{l}\text { It was hypothesized that when age was included as } \\
\text { a moderator, the accelerometer, daily diary, and } \\
\text { GLTEQ data would be associated similarly across } \\
\text { younger adults (Group 1) and middle-aged and } \\
\text { older adults (Group 2) for intensity (moderate-to- } \\
\text { vigorous, mild, and sedentary), duration, and } \\
\text { frequency. }\end{array}$ & Largely supported \\
\hline $\begin{array}{l}\text { Research } \\
\text { Question } 3\end{array}$ & $\begin{array}{l}\text { Does sex influence agreement for intensity, } \\
\text { duration, and frequency across the accelerometer, } \\
\text { daily diary, and GLTEQ? }\end{array}$ & \\
\hline
\end{tabular}




\begin{tabular}{cll}
\hline Hypothesis 3a & $\begin{array}{l}\text { It was hypothesized that the relations for the } \\
\text { accelerometer, daily diary, and GLTEQ data would } \\
\text { be associated similarly across males and females for } \\
\text { intensity (moderate-to-vigorous, mild, and } \\
\text { sedentary), duration, and frequency. }\end{array}$ & Partially supported \\
\hline Research & $\begin{array}{l}\text { Does intrinsic motivation to by physically active } \\
\text { Question 4 } \\
\text { influence agreement for intensity, duration, and } \\
\text { frequency across the accelerometer, daily diary, and } \\
\text { GLTEQ? }\end{array}$ & \\
\hline Hypothesis 4a & $\begin{array}{l}\text { It was hypothesized that for intensity (moderate-to- } \\
\text { vigorous, mild, and sedentary), duration, and }\end{array}$ & $\begin{array}{l}\text { Largely not } \\
\text { supported }\end{array}$ \\
& $\begin{array}{l}\text { frequency, the associations between the } \\
\text { accelerometer, daily diary, and GLTEQ would }\end{array}$ & \\
& demonstrate a medium level of agreement $(.29<\mathrm{r}<$ & \\
& 49) when participants are intrinsically motivated to & \\
be physically active. & \\
\hline
\end{tabular}

